\section{ORAL PRESENTATIONS ABSTRACTS}

\section{0-001}

Thymoquinone and Sorafenib as a therapeutic combination in liver cancer: In vitro and in vivo

Eray Metin Guler, Abdurrrahim Kocyigit

Bezmialem Vakif University, School of Medicine, Department of Medical Biochemistry, Turkey

OBJECTIVES:Hepatocellular carcinoma (HSC) is the most common primary malignant tumor of the liver originating from hepatocytes. The aim of this study is to investigate the antitumor effects of chemotherapeutic agent sorafenib and N.sativa's active substance thymoquinone against in vivo and in vitro hepatocellular carcinoma.

MATERIALS and METHODS:Cytotoxicity, genotoxicity, apoptosis, intracellular ROS, intracellular glutathione and mitochondrial membrane potential were measured in Luc-transfected hepatocellular carcinoma cells. Cells were given to nude mices by zenograft method. TQ, sorafenib, and combined therapy reduced tumor size after the 4 week treatment. Tumor size were measured with an IVIS imaging device and caliper.

RESULTS:In vitro dose-dependent thymoquinone and sorafenib have cytotoxic genotoxic, apoptotic and ROS-producing effects, both individually and in combination. In vivo study, combine therapy was found to be more effective than mono therapies in vivo hepatocellular carcinoma, which was formed by the xenographic method. While tumor size, inflammation, oxidative stress decreased, While tumor size, inflammation, oxidative stress decreased, antioxidantive markers and thiol levels increased. CONCLUSIONS:Our results showed that, thymoquinone has anticancer properties in vivo and in vitro. It has been shown to be more effective at lower doses when used with routine therapy.

Keywords: Thymoquinone, Sorafenib, hepatocellular carcinoma, IVIS

\section{O-002}

Investigation of type I collagen and MMP-2 changes in mandibular bone tissue in natural development

Velid Unsal ${ }^{1}$, Mustafa Çiçek ${ }^{2}$

${ }^{1}$ Department of Nutrition and Dietetics, Faculty of Health Science, Mardin Artuklu University, Mardin, Turkey

${ }^{2}$ Department of Anatomy, Faculty of Medicine, Kahramanmaras Sütcü Imam

University, Kahramanmaras, Turkey.

OBJECTIVES:Mandibular bone, which is a part of the masticatory system, changes in histology and molecular structure based on the age and gender of an individual. The masticatory system develops with age and it affects all oral and temporomandibular joint disorders. In this study, we have aimed to examine the effects that aging has on the changes on type I collagen, which exists in the bone tissue and provides its matrix and its durability, and matrixmetalloproteinase- 2 (MMP-2).

MATERIALS and METHODS:14 Balb / C species white mice were used in the study. Animals were divided into two groups of seven, based on whether they are young or old. Mandibular bone tissue homogenate was prepared for biochemical analyses and mandibular bone tissue was obtained for histological evaluations. After routine histological follow-up, the tissues were embedded in paraffin. 4-5 $\mu \mathrm{m}$ thick sections were taken from paraffin-embedded tissues and hematoxylin-eosin, Type I collagen and MMP-2 immunohistochemical stainings were performed

RESULTS:Ca+2, ALP ve calcitonin levels were decreased in the aging-based bone tissue homojenate analyses that were performed and TNF- $\alpha$ and PTH levels were significantly increased. Type I collagen and MMP-2 immunoreactivity in elderly mice showed a significant decrease in comparison to young mice.

CONCLUSIONS:As a result, aging causes a decrease in the amount of bone formed in the bone reconstruction cycle due to the decrease in the osteoblast support and the increases osteoclastic activity

Keywords: Type I collagen, MMP-2, Calcium, Calcitonin, TNF- $\alpha$

\section{O-003 \\ Induction of APAF-1 and TRAIL by bilberry tea in HCT-116 colon cancer cell line}

Burak Durmaz ${ }^{1}$, Latife Merve Oktay ${ }^{2}$, Hikmet Memmedov ${ }^{1}$, Nur Selvi Günel ${ }^{2}$ Hatice Kalkan Yıldırım³, Eser Yıldırım Sözmen

${ }^{1}$ Department of Medical Biochemistry, Ege University Faculty of Medicine, İzmir, Turkey

${ }^{2}$ Department of Medical Biology, Ege University Faculty of Medicine, İzmir, Turkey

${ }^{3}$ Department of Food Engineering, Ege University Faculty of Engineering, İzmir, Turkey

OBJECTIVES:In this study, it was aimed to determine the effect of bilberry tea samples on the markers of the intrinsic and extrinsic pathways of apoptosis in the HCT-116 colon cancer cell.

MATERIALS and METHODS:Bilberry tea in different infusions and boiling (1 min $3 \mathrm{~min}, 5 \mathrm{~min}, 7 \mathrm{~min}, 10 \mathrm{~min}$ ) were prepared and phenolic levels were determined by LC MS / MS technique. The highest phenolic content was determined in tea samples of seedless fruits for 5 min boiling, so this product was chosen for in vitro study. Cytotoxicity and viability tests were performed by adding WST- 8 solution. Intrinsic and extrinsic pathways of Apoptosis were assessed by determining the TRAIL, APAF-1,Cytochrome-c, Caspase -3,-8,-9 levels in HCT-116 colon cancer cell line. RESULTS:Cytotoxicity studies in cell culture were conducted using $50-10 \mu \mathrm{g} / \mathrm{ml}$ of bilberry tea samples which was prepared at a concentration of $5 \mathrm{~g} / 10 \mathrm{ml}$. The levels of APAF-1, TRAIL and Cytochrome-c were significantly higher in bilberry added cell culture than the control cells. Other markers (caspase $-3,-8,-9$ levels) did not show any significant change compared to control cells

CONCLUSIONS:It is concluded that bilberries induced TRAIL, APAF-1, Cytochrome-c and consequently induced both intrinsic and extrinsic pathways of apoptosis.

Keywords: APAF-1, TRAIL, Bilbery, HCT-116, Colon cancer

O-004

Induction of apoptosis and cell cycle arrest by pomegranate extract and tangeretin in the rat mammary carcinogenesis

Husevin Fatih Gul ${ }^{1}$, Necip Ilhan ${ }^{2}$, Nevin Ilhan² ${ }^{2}$ Ibrahim Hanifi Ozercan ${ }^{1}$ Department of Medical Biochemistry, Faculty of Medicine, Kafkas University, Kars, Turkey

${ }^{2}$ Department of Medical Biochemistry, Faculty of Medicine, Firat University, Elazıg, Turkey

${ }^{3}$ Department of Medical Pathology, Faculty of Medicine, Firat University, Elazıg, Turkey

OBJECTIVES:The present study investigated the potential chemoprevention effects of Pomegranate extract $(\mathrm{P})$ and Tangeretin $(\mathrm{T})$, both alone and in combination, on the apoptosis and cell cycle in 7,12-dimethylbenz [a] anthracene (DMBA)-induced rat mammary carcinogenesis.

MATERIALS and METHODS:Sprague Dawley female rats $(n=56)$ were randomly divided into 8 groups. Group I was control, Group II, III and IV were treated with $\mathrm{P}, \mathrm{T}$ and $\mathrm{P}+\mathrm{T}$ respectively. Group $\mathrm{V}$ was DMBA-induced (a single dose of $60 \mathrm{mg}$ / $\mathrm{kg}$ body) weight breast cancer-bearing rats. Group VI, VII, VIII were designed as the chemopreventive treatment groups and were composed of D+P, D+T, D+P+T groups, respectively. The presence of the breast tumour tissue was demonstrated with histopathological examinations. In the breast tissue samples, the expressions leves of p53, Bax, Bcl-2 and cyclin D1 proteins acting on apoptosis and cell cycle were performed by western blot analysis.

RESULTS:According to histopathological evaluations, it was determined that most $(90 \%)$ of the tumours created were invasive ductal carcinoma. While 553 and Bax expressions of pro-apoptotic markers significantly decreased in the DMBA group compared to the control group, it was observed that Bcl-2 and cyclin D1 expressions significantly increased. It was observed that p53 and Bax expressions significantly increased in both $\mathrm{D}+\mathrm{P}$ and $\mathrm{D}+\mathrm{P}+\mathrm{T}$ groups compared to the DMBA group. Cyclin D1 expressions were determined to significantly decrease only in the D+T group. CONCLUSIONS:Our study results have shown that the combined administration of Pomegranate extract and Tangeretin may be more beneficial in preventing breast cancer. Keywords: Breast cancer, Apoptosis, Cell cycle arrest, Pomegranate extract, Tangeretin 


\section{O-005}

Preparation of magnetic nanoparticle coated glutaraldehyde to reduce toxic effects of idarubicin and its effect on HL60 cell line

Hasan Ulusal $^{1}$, Fatma Ulusal ${ }^{3}$, Mehmet Tarakçıoğlu ${ }^{1}$, Seyithan Taysi ${ }^{1}$,

Bilgehan Güzel

${ }^{1}$ Department of Biochemistry, Gaziantep University, Gaziantep, Turkey

${ }^{2}$ Department of Chemistry, Cukurova University, Adana, Turkey

${ }^{3}$ Department of Biochemistry, Gaziantep University, Gaziantep, Turkey,

Department of Chemistry, Cukurova University, Adana, Turkey

OBJECTIVES:Anthracyclines (doxorubicin, daunorubicin and idarubicin) are very effective chemotherapeutic drugs to treat many cancers; however, they cannot distinguish between healthy cells and cancer cells and cause serious side effects and systemic toxicity. Furthermore, one of the major problems is that the drugs which are being used cannot be used efficiently because of their low half-life time and low stability. In recent years, studies have focused on magnetic nanoparticles (MNP) which are capable of carrying drugs to overcome these shortcomings. The aim of this study was to immobilize idarubicin (IDA) to glutaraldehyde-coated MNPs, to prepare a drug with high stability and low toxicity levels.

MATERIALS and METHODS:MNPs were prepared and coated with glutaraldehyde, IDA was immobilized and its activity in HL-60 cell line was examined. All of the materials were characterized by various measurements, including XRD,TEM, SEM and UV-Vis. Idarubicin loaded MNPs were administered to HL60 cell line at different doses, and MTT and ATP cell viability analyzes were performed and compared to free idarubicin.

RESULTS:The in-vitro cytotoxicity results showed that the IC50 value of IDAMNPs was 13-folds lower than that of free IDA solution in HL60 cell line (IC50: $0,029 \mu \mathrm{M}$ for IDA-MNPs and $0,396 \mu \mathrm{M}$ for free IDA). In addition, analyzes showed that idarubicin was bound to MNP system by $54 \%$.

CONCLUSIONS:The results of this study showed that MNP-induced idarubicin is effective in eliminating cancer cells even at doses 13 times lower. So these results show promising effects in cancer treatment.

This study was supported by TUBITAK BIDEB 2218 .

Keywords: Magnetic nanoparticles, HL60 cell line, glutaraldehyde, idarubicin

\section{O-006}

The effects of overexpression of acetylcholinesterase on amyloid precursor protein and $\beta$-secretase-1 levels in Hs766T cells

Kevser Biberoglu, Seda Onder, Ozden Tacal

Department of Biochemistry, School of Pharmacy, Hacettepe University, Ankara, Turkey

OBJECTIVES:Acetylcholinesterase (AChE) plays a key role in catalytic hydrolysis of acetylcholine. It has known that acetylcholine can cause angiogenesis, migration and proliferation of cancer cells via activating the nicotinic acetylcholine receptor. Intensive research has indicated that AChE-R (readthrough isoform) is involved in proliferation, whereas AChE-T (tailed isoform) plays a role in apoptosis. A recent study has shown that AChE-T has potent anti-tumor effects and causes apoptosis of gastric cancer cells in-vitro and in-vivo. With the discovery of non-classical functions of $\mathrm{AChE}$ on cancer cells, the proteins that interact with $\mathrm{AChE}$ have become remarkable. Inhibiting the expression of amyloid precursor protein (APP) or $\beta$-secretase-1 (BACE1) is one of the therapeutic strategies due to their positive effects on cancer cell proliferation. In this study, we wonder whether AChE-T has any effects on APP and BACE1 expression in Hs766T pancreatic cancer cells.

MATERIALS and METHODS:Hs766T cells were transiently transfected with pGS-AChE-T plasmid, using lipofectamine-2000. To check transfection efficiency, AChE activity was assayed spectrophotometrically. After 48 hours of transfection, the levels of APP and BACE1 in cell lysates were analyzed using Western Blot.

RESULTS:We observed a significant decrease in both APP and BACE1 levels in transfected cells compared to vehicle-treated cells. Mature and immature APP levels were reduced by $60 \%$ and $68 \%$, respectively whereas mature and immature BACE1 levels were reduced by $30 \%$ and $71 \%$, respectively.

CONCLUSIONS: AChE-T reduces the levels of BACE1 and APP in Hs766T cells therefore it may show anti-cancer effects.

Supported by a grant from Hacettepe University Scientific Research Projects
Coordination Unit (HUBAB, TSA-2017-13929)

Keywords: acetylcholinesterase, $\beta$-secretase-1, amyloid precursor protein, pancreas cancer

\section{O-007 \\ Genome-wide CRISPR-Cas9 screening for identification of cancer essential genes in malignant pleural mesothelioma}

\section{Ece Cakiroglu, Ozlem Silan Coskun, Serif Senturk}

Izmir International Biomedicine and Genome Institute, Dokuz Eylul University, Izmir, Turkey; Izmir Biomedicine and Genome Center, Izmir, Turkey

OBJECTIVES:Malignant pleural mesothelioma (MPM), which accounts for 80$90 \%$ of all mesothelioma cases, is a rare cancer with an increasing incidence and low survival rates. Existing treatment options are limited to chemotherapy with a low success rate. Therefore, novel targeted therapies are needed. In this study, we applied genome-wide negative selection CRISPR-Cas9 screening to identify cancer cell essential genes in MPM cell lines.

MATERIALS and METHODS:To obtain stable Cas9 expression we transduced 3 different MPM cancer cell lines and 1 normal epithelial cell line with lentiCas9EGFP vector. FACS was performed to obtain and select clonal sublines with highest Cas9 expression. Competition assay and T7E1 assay were performed for functional characterization of selected clones. Brunello gRNA library was amplified and lentiviral particles were produced. Selected clones were transduced with Brunello gRNA library at MOI 0.3-0.5 and selected with puromycin and were cultured for 14 doublings.

RESULTS:We obtained clonal sublines showing permanent Cas9 nuclease expression. Selected clones with highest Cas9 expression were functionally characterized and were screened by transducing with whole genome Brunello gRNA library. Fold coverage of $>400 \mathrm{x}$ was achieved following transduction. CONCLUSIONS:Although whole genome CRISPR-Cas9 screening has some challenges due to the usage of high volume of cell cultures. While the risk of skewing of the composition of the final recovered DNA is high, CRISPR-Cas9 screening is still a powerful tool for obtaining essential genes and druggable targets in cancer. This study was supported by the Scientific and Technological Research Council of Turkey (TUBITAK) (Project number: 117Z227)

Keywords: CRISPR-Cas9 screening, brunello library, malignant pleural mesothelioma

\section{O-008}

The importance of serum hyaluronidase measurement in discrimination of patients with prostate cancer and benign prostatic hyperplasia

Zeynep Adıyaman Koçer ${ }^{1}$, Elmas Öğüş ${ }^{1}$, Tuba Özgün' ${ }^{1}$, Koray Ağras², Veysel Bayburtluoğlu ${ }^{2}$, Doğan Yücel ${ }^{1}$

${ }^{1}$ Health Sciences University Ankara Health Application and Research Center, Department of Medical Biochemistry, Ankara

${ }^{2}$ Health Sciences University Ankara Health Application and Research Center, Department of Urology, Ankara

OBJECTIVES:The aim of this study was to investigate the ability of serum HYAL activity and mass concentration to distinguish prostate cancer (PC) from benign conditions.

MATERIALS and METHODS:Our study included age-matched 37 newly diagnosed PK, 72 benign prostatic hyperplasia (BPH), 53 chronic prostatitis $(\mathrm{CrP})$ patients according to biopsy results and 49 control patients. Other cancers, liver disease, rheumatologic diseases, collagen tissue disease and dermatological disorders that could increase serum HYAL levels were excluded. Morgan-Elson colorimetric method was used to measure serum HYAL activity (sHYALa). Serum HYAL concentration (sHYALc) was determined by an ELISA method. Biopsy results were used for evaluation of clinical performance.

RESULTS:sHYALa, sHYALc and total PSA levels were found to be significantly higher in PK patients compared to control and benign patients $(\mathrm{p}<0.05)$. In all groups, there was a relatively weak positive correlation between sHYALa and PSA ( rho $=0.405, \mathrm{p}<0.05, \mathrm{n}=141)$; sHYALc and PSA $(\mathrm{rho}=0.344, \mathrm{p}<0.05, \mathrm{n}=88)$. sHYALa and sHYALc was found to be significantly higher in PK patients with PSA values in gray zone $(4-10 \mu \mathrm{g} / \mathrm{L})$ compared to other benign patient groups $(\mathrm{p}<0.05)$. In ROC analysis, AUC for sHYALa, sHYALc and PSA were 0.866 ; 
0.826 and 0.813 , respectively. Sensitivity and specificity were found for sHYALa, sHYALc and PSA as $88 \%, 71 \% ; \% 82, \% 89$ and $79 \%, 71 \%$, respectively. CONCLUSIONS:Combining sHYALa or sHYALc with PSA, physical examination and ultrasonography data may be useful in the evaluation of PK patients.

Keywords: Hyaluronidase, Prostate, Cancer

\section{O-009 \\ Antioxidant and anti-denaturation activities of asparagus horridus grows in North Cyprus}

$\underline{\text { Duygu Gençalp }}^{1}$, Ergül Mutlu Altundağ ${ }^{1}$, Cahit Özbilenler ${ }^{2}$

Namık Refik Kerkuklu²

${ }^{1}$ Department of Medical Biochemistry, Eastern Mediterranean University, Famagusta, North Cyprus

${ }^{2}$ Department of Chemistry, Eastern Mediterranean University,Famagusta, North Cyprus

OBJECTIVES: Asparagus horridus is an edible plant known as "Ayrelli" in North Cyprus. There is a huge information gap in literature about this plant. The purpose of the research was to determine the antioxidant and anti-denaturation activities of the Asparagus horridus.

MATERIALS and METHODS:In this study, soxhlet extraction was used to obtain the extract from air-dried Asparagus horridus plant. We conducted a 1,1-diphenyl-2-picrylhydrazyl (DPPH), total flavonoid content (TFC),Ferric reducing activity and total phenolic content (TPC) tests to determine the antioxidant activity with using standard methods. Protein degredation assay was performed to determine the anti-denaturation activity of Asparagus horridus extract.

RESULTS:The DPPH test of Asparagus horridus methanol extract showed an increase of DPPH scavenging activity from $27.71 \%(p<0.0001)$ to $49.69 \%$ $(\mathrm{p}<0.0001)$ with the extract dose from 15 to $25 \mathrm{mg} / \mathrm{ml}$.Total Phenolic Content of the extract was determined as $140.68(\mathrm{p}<0.01)$ to $167.61(\mathrm{p}<0.01) \mathrm{mg} / \mu \mathrm{g}$ equivalent of gallic acid with the extract dose from 15 to $25 \mathrm{mg} / \mathrm{ml}$. Beside that Total Flavonoid Content was obtained as $119.72(\mathrm{p}<0.00001)$ to 273.5 $(\mathrm{p}<0.00001) \mathrm{mg} / \mu \mathrm{g}$ equivalent of quercetin with the extract dose from 10 to 25 $\mathrm{mg} / \mathrm{ml}$.Ferric reducing activity varied from $0.36(\mathrm{p}<0.001)$ to $1.27(\mathrm{p}<0.0001)$ $\mathrm{mg} / \mathrm{\mu g}$ equivalent of $\mathrm{FeSO} 4$ with the extract dose from 10 to $25 \mathrm{mg} / \mathrm{ml}$. When anti-denaturation activity of Asparagus horridus extract was checked, is was found that the extract exhibited the highest inhibitory activity at $25 \mathrm{mg} / \mathrm{ml}$ as \% $29.41 \pm 0.34$

CONCLUSIONS:Consequently, these results showed that the methanol extract of Asparagus horridus plant grows North Cyprus has important antioxidant and anti-denaturation potential.

Keywords: Asparagus horridus, North Cyprus, antioxidant, anti denaturation

\section{O-010}

CA125 test request ratio in male patients

\section{Muammer Yücel, Huriye Erbak Yilmaz}

Department of Clinical Biochemistry, İzmir Atatürk Education and Research Hospital, İzmir, Turkey

OBJECTIVES:Cancer Antigen 125 (CA125) test is one of the most commonly studied tumor markers in clinical biochemistry laboratories. Low sensitivity and specificity of CA125 restrict the clinical use of it. In addition to ovarian cancer, it may increase in tumor-related diseases of serous membranes and a number of benign conditions.CA125 test can be ordered by all clinicians in our hospital. We examined CA125 test orders in detail in the 6-month period and detected unnecessary test orders. MATERIALS and METHODS:CA125 test was performed by chemiluminescence method on Advia Centaur XPT(Siemens) analyzer in our laboratory. CA125 which were analyzed between January - July 2019 period were examined from laboratory information system (ALIS, Ventura)(Reference Range: 0 - $35 \mathrm{U} / \mathrm{mL}$ ). RESULTS:In the 6-month period, 5,635 CA125 tests were performed which 1,356 of them belong to male patients(24\%). In those patients, $144(10 \%)$ results of the ordered CA125 were found above the reference range(min:36, Max:4,463, median:87.5). Oncology(35\%) and Internal Medicine(30\%) clinics were having most common orders of CA125. CONCLUSIONS:Unnecessary tests increase the laboratory workload and high costs. The use of tumor markers for screening in patients having no symptoms is one of the most common reason of the unnecessary test ordering. The main usage of CA125 test is non-mucinous ovarian carcinoma. Therefore, except in exceptional cases, it should not recommended for male patients.Departmentbased and/or gender-based test ordering restrictions through the hospital information system may prevent unnecessary test ordering, such as the order of CA125 for male patients. In addition a pop-up message can be created during the clinician orderings.

Keywords: CA125, unnecessary test request, tumor markers

O-011

Evaluation of tumor marker tests in a hospital setting

\section{Muzaffer Katar}

Department of Cliniical Biochemistry, School of Medicine, Tokat Gaziosmanpasa University, Tokat, Turkey

OBJECTIVES:Early diagnosis and treatment of oncological diseases is extremely important. In this study, we aimed to evaluate tumor marker requests of our hospital and investigate the presence of improper use.

MATERIALS and METHODS:Evaluation of the tumor markers (CEA, CA 15-3, CA 19-9 and CA 125 ) performed by the biochemistry laboratory of Tokat Gaziosmanpasa Research and Application Hospital between 01.01.2018 and 31.12.2018 was accomplished. Our parameters were divided into subgroups according to being within and above the reference ranges. The clinical application of tumor markers can be divided into 4 groups: screening, diagnostic confirmation, prognosis, and monitoring of recurrence. Internal Medicine, Gastroenterology, Endocrine Diseases, Chest Diseases, General Surgery, Gynecology and Obstetrics and Medical Oncology have made requests.

RESULTS:Total requests were 1420 for CEA, 671 for CA15-3, 868 for CA 19-9, and 585 for CA 125. A significant difference between genders for CEA and CA 125 was determined ( $<<0.001$ and $\mathrm{p}$ : 0.033 , respectively). 312 (22\%) of CEA, $202(30.1 \%)$ of CA 15-3, 204 (23.5\%) of CA 19-9, and $113(19,3 \%)$ of CA 125 requests were above the reference ranges. Significant positive correlations were determined between age and tumor markers of CEA, CA 15-3, and CA 19-9 (r: $0,262, p<0,001 ; \mathrm{r}: 0,096, \mathrm{p}: 0,013 ; \mathrm{r}: 0,090, \mathrm{p}: 0,008$; respectively). Preliminary diagnoses were nonspecific pain, acute vaginitis, anemia, anxiety disorder, dyspepsia, neoplasm and thyroid disorders.

CONCLUSIONS:This study shows that many outpatient clinics have made excessive amount of tumor marker requests incompatible with preliminary diagnosis suggesting overutilization. This situation causes cost and workload. Keywords: Inappropriate Test Request, Oncology, Neoplasm Tumor Markers

O-012

Detection of preanalytical errors in blood gas analysis

Serap Çuhadar ${ }^{1}$, Hayat Özkanay Yörük ${ }^{1}$, Mehmet Hicri Köseoğlu ${ }^{1}$, Kaan Katırcioğlu ${ }^{2}$

${ }^{1}$ Department of Biochemistry, Izmir Katip Çelebi University, Atatürk Training and Research Hospital, Izmir, Turkey

${ }^{2}$ Department of Anesthesiology and Reanimation, Izmir Katip Çelebi University, Atatürk Training and Research Hospital, Izmir, Turkey

OBJECTIVES:Blood gas analysis is an urgent test needs to be studied in a short time interval for its preanalytical instability and there is still no consensus about the storage temperature. The aim of this study was to determine the effect of air bubbles in the blood gas injectors and different temperature conditions on the results.

MATERIALS and METHODS:Arterial blood was collected from 20 patients in intensive care unit into lithium heparin syringes from their catheter. The samples were grouped as; room temperature, room temperature plus air bubble, +4 degrees, and +4 degree plus air bubble. Blood gas analyses were performed by a potentiometric method using a blood gas analyzer ABL 800 (Radiometer,Copenhagen,Denmark) within 5 minutes (baseline) and at 30, 60, 90 and 120 minutes. Results were compared with baseline statistically with paired 
samples t-test or Wilcoxon signed rank test with post hoc Bonferroni correction $(\mathrm{p}<0.0125)$, and evaluated clinically according to the desirable bias.

RESULTS:PCO2 results were increased significantly in all study groups. PO2 levels were unaffected at room temperature up to $60^{\circ}$, but found as increased at 30 ' when cooled. $\mathrm{PH}$ levels were all in acceptable limits. $\mathrm{HCO} 3$ was stable up to 90 ', and $\mathrm{SaO} 2$ levels were affected less than $1 \%$ in all groups.

CONCLUSIONS:Cooling the arterial blood sample in plastic syringe is inappropriate for $\mathrm{pO} 2$ analysis, however, it was found as stable up to 60 ' at room temperature. In conclusion, room temperature is better than cooling samples in plastic syringes for arterial blood gas analysis.

Keywords: blood gas, preanalytical errors

\section{O-013}

The Effect of Hemolysis and Storage Conditions on Insulin Stability

Didem Barlak Keti, Sabahattin Muhtaroglu

Department of Medical Biochemistry, Erciyes University, Kayseri, Turkey

OBJECTIVES:Biochemical or spectrophotometric measurements are known to be more affected by hemolysis when compared to immunochemical analysis. This situation can often lead to less consideration on immunochemical assays. Threshold values at which hemolysis affects immunochemical tests are indicated in our kit inserts, but there is no value related to insulin. Therefore, the aim of this study is to determine the hemolysis threshold for insulin and the effect of storage conditions on serum insulin stability.

MATERIALS and METHODS:Serum pools were formed from the samples of the routine laboratory. Serum samples of equal volume were transferred to seven tubes. The tubes were designed as only serum in the first tube, serum + assay diluent in the second tube, and serum + hemolysate in the 3-7 tubes which correspond to $50,100,200,400$ and 800 , respectively hemolysis index. In addition, insulin levels were measured in the patient samples with $<20(n=10)$, 20-50 $(n=10), 50-100(n=10)$ and 100-200 $(n=10)$ hemolysis index immediately and after 8 hours at room temperature.

RESULTS:Negative bias was detected as $10 \%$ in the samples with below 200 $\mathrm{mg} / \mathrm{dL}$ hemolysis index which were analysed immediately after centrifugation. Negative bias was determined as $<10 \%, 27.6 \%$ and $29.5 \%$ in the samples $<20$, 20-50 and 50-100 hemolysis index, respectively which stayed for 8 hours at room temperature.

CONCLUSIONS:Hemolysis index should be considered when reporting insulin levels. Insulin analysis is not suitable for hemolysed serum samples that have waited 8 hours at room temperature.

Keywords: Hemolysis, insulin, stability

\section{O-014}

The effect of hemolysis and storage conditions on insulin stability

\section{Merve Sibel Güngören, Yahya Rauf Laleli}

Duzen Laboratories Group, Ankara, Turkey

OBJECTIVES:Interferences are the most serious limitations of immunoassays which are one of the most common measurement methods in clinical chemistry. According to the measurement principle of immunoassay, sources of interference may vary from antibodies, to vitamins, drugs and endogenous molecules. Unconjugated estriol (uE3) assay is an important component of second trimester screening and negative interference of $\mathrm{uE} 3$ assay may lead to false Down Syndrome and even Smith Lemli Opitz Syndrome risk. The aim of this case report is to present a case series of 70 patients with falsely low uE3 results from Beckman Coulter DxI 800 instrument.

MATERIALS and METHODS:Increase in number of low uE3 results $(<0.3$ $\mathrm{ng} / \mathrm{mL}$ ) led us to confirm the results. We started to dilute samples $1 / 2$ and $1 / 5$ any sample with the result below $0.3 \mathrm{ng} / \mathrm{mL}$. Recovery results above $150 \%$ were considered as interfered samples. 1500 patient samples were screened to detect negative interference in uE3 assay within two years period. Samples with suspicion of interference were furtherly investigated in Beckman Coulter Laboratories (Marseille, France).

RESULTS:70 samples were found to be confirmed to be affected by interference which was cleared by scavenger ALP. Interfered results were below $0.56 \mathrm{ng} / \mathrm{mL}$. Recovery results varied from 150 to $600 \%$.
CONCLUSIONS:This is the first case series of negative $\mathrm{uE} 3$ interference. It was speculated that scavenger ALP molecules bind to endogenous ALP antibodies. ALP is the conjugate of manufacturer's $\mathrm{uE} 3$ kit and any molecule interfering with ALP affects uE3 kit.

Keywords: Interference, immunoassay, uE3

\section{O-015}

\section{What if all is well except insulin: A macroinsulin case report}

Cevdet Züngün, Merve Sibel Güngören, Yahya Rauf Laleli Duzen Laboratories Group, Ankara, Turkey

OBJECTIVES:Macroinsulin is a larger molecule of insulin comprised of insulin and insulin antibody (IA). This phenomenon is rare and generally due to exogenous insulin therapy. The aim of this case report is to present a patient with macroinsulin who has never had exogenous insulin.

MATERIALS and METHODS:A 75-year old male was admitted to our laboratory for routine check-up. All test results including fasting and postprandial glucose levels and HbAlc were within age-specific reference intervals, except for fasting and postprandial insulin levels $(110.80 \mu \mathrm{IU} / \mathrm{mL}$ and $163.80 \mu \mathrm{IU} / \mathrm{mL}$, respectively). He had no history of insulin resistance or diabetes mellitus. His fasting and postprandial C-peptide, islet antibody, glutamic acid decarboxylase antibody levels were normal However, insulin antibody level was found to be eight fold higher than the upper limit. To prove the reason for elevated insulin, polyethylen glycol (PEG) solution is used to precipitate the insulin-IA complexes and serum insulin was re-analysed from the supernatant. Two different patients' sera with high insulin levels were also treated with PEG as control study.

RESULTS:Result of insulin in PEG-treated patient sample has been found to be decreased from 110.80 to $19.20 \mu \mathrm{IU} / \mathrm{mL}(\sim 80 \%)$ of the first insulin measurement. Insulin results of the PEG-treated control sera were found to be similar with native sera.

CONCLUSIONS:Discrepantly high results of insulin with normal C-peptide has to be furtherly investigated with IA measurement and re-analysis of from PEGtreated serum. Insulin-IA complexes thought to be responsible for the elongated half-life of insulin in the circulation.

Keywords: Insulin, macroinsulin, insulin antibody, antigen-antibody complex, interference

\section{O-016}

Comparison of biochemical analytes in different blood collection tubes and evaluation of stability

Inanc Karakoyun ${ }^{1}$, Fatma Demet Arslan ${ }^{1}$, Selin Onur ${ }^{1}$, Yasemin Kilic Ozturk ${ }^{2}$, Hulya Parildar ${ }^{2}$, Banu Isbilen Basok ${ }^{1}$

${ }^{1}$ University of Health Sciences, Tepecik Training and Research Hospital, Department of Medical Biochemistry, Izmir, Turkey

${ }^{2}$ University of Health Sciences, Tepecik Training and Research Hospital, Department of Family Medicine, Izmir, Turkey

OBJECTIVES:In this study, we compared 3 different clot-activator gel tubes to a glass reference tube and evaluated the effect of storage time on 31 different biochemical analytes.

MATERIALS and METHODS:Blood samples were collected in 4 types of tubes: an additive- and gel-free glass tube and three different clot-activator tubes containing gel (Samplix, Vacuette, and Vacutainer). In addition to comparison with the glass tube, stability analyses were performed in Samplix, Vacuette, and Vacutainer tubes after storage for 48 hours at $+4^{\circ} \mathrm{C}$. Clinically important differences were evaluated using the Ricos desirable specifications for bias based on biological variation. RESULTS:Clinically important differences were found for albumin(bias $\%$, -2.39), sodium(-0.29), potassium(2.35) and magnesium(2.78) in Samplix; for sodium(-0.27), potassium (2.82), lactate dehydrogenase (4.47) and magnesium(2.46) in Vacuette; and for calcium(-1.56), chloride(0.66), potassium (3.54), lactate dehydrogenase $(9.11)$ and sodium $(0.38)$ in Vacutainer. At the end of the 48 hours, analytes that demonstrated instability were albumin(-3.13), chloride(1.01), potassium(2.69), sodium(0.54), and total protein (1.95) in Samplix; albumin(-6.45), $\mathrm{Cl}(1.11)$, potassium(2.06), and sodium(0.84) in Vacuette; and albumin(-4.57), calcium(1.28), chloride(0.64), free T3(-8.87), glucose(2.76), potassium(2.19), sodium(0.65), and total protein(2.15) in Vacutainer. 
CONCLUSIONS:Various blood collection tubes (BCTs) in different contents may cause clinically important differences in the test results. Therefore, each laboratory should verify the reference range transfer or create its own reference range before using a new BCT. It should be also considered that in cases where analysis cannot be completed immediately after blood sampling, not all clinical chemistry or immunological test analytes can maintain their stability in BCTs up to 48 hours

Keywords: Blood collection tube, Serum, Stability

\section{O-017}

Elevated high sensitivity troponin in the absence of coronary artery disease: A case report

\section{Feyza Yağmur Tekeli ${ }^{1}$, Seçkin Özgür Tekeli ${ }^{1}$, Ahmet Genç $^{2}$}

${ }^{1}$ Department of Biochemistry,Antalya Education and Research Hospital, Antalya ${ }^{2}$ Department of Cardiology,Antalya Education and Research Hospital, Antalya

OBJECTIVES:The Joint European Society of Cardiology/American College of Cardiology committee for the redefinition of myocardial infarction (MI) states that troponins are the preferred cardiac marker for detecting myocardial injury. There are a few important non-ACS causes of cardiac troponin elevation that require immediate attention and treatment.

MATERIALS and METHODS:This is a case of a 56-year-old female came to the emergency department complaining of shortness of breath and lightheadedness. She denied any chest pain, nausea or vomiting. ECG showed sinus rhythm with minimal ST-T deviation, and chest X-ray showed no acute process. Routine biochemistry tests were within normal limits, except for hsTnT of $22.05 \mathrm{ng} / \mathrm{L}$ ( $(0-14 \mathrm{ng} / \mathrm{L})$ and $\mathrm{Hgb}$ of $7,1 \mathrm{~g} / \mathrm{dL}(12,5-16 \mathrm{~g} / \mathrm{dL})$. Repeat test of hsTnT ( after 1 $\mathrm{hr}$, and $6 \mathrm{hr}$ ) respectively; $30 \mathrm{ng} / \mathrm{L}$ and $25 \mathrm{ng} / \mathrm{L}$

RESULTS:She was diagnosed to have anemia and was given one unit of blood. Anemia was considered to be the cause of elevated troponin levels. Severe aortic stenosis was detected in the echo performed 6 months later, ECG showed sinus rhythm with ventricular HPT findings. NT Pro-BNP was found to be $1952 \mathrm{pg} / \mathrm{mL}$ (significant $>900 \mathrm{pg} / \mathrm{mL}$ )

CONCLUSIONS:Coexistence of aortic stenosis and anemia explains increased troponin values. This case report confirms that BNP test can be used as an early marker in cardiac function monitoring. It also shows that moderate elevations in troponin levels can be an indicator of non cardiac MI The importance of cardiac function tests should be discussed for more frequent follow-up of rheumatic valve patients.

Keywords: troponin, anemia, aort stenosis, proBNP

\section{O-018}

Serum separation problem on gel tubes: Is it a problem or a clue of some clinical conditions?

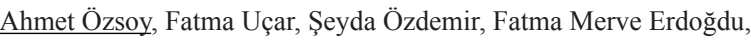
Ali Yalçındağ

Department of Biochemistry,University of Health Sciences,Dışkapı Yıldırım Beyazit Training and Research Hospital,Ankara, Turkey

OBJECTIVES:Serum separator tubes which contain separator gels are widely used by many laboratories. The gel forms a physical barrier between the cellular elements of the blood and serum. We report a case with serum separation problem in a patient hospitalized for recurrent epistaxis and regulation of hypertension. MATERIALS and METHODS:The patient was 85 years old female followed in internal medicine department. The blood sample was collected into a BD Vacutainer SST II Advance (Becton Dickinson, NJ, USA) containing serum separator gel tube. After centrifugation, pipetting error alerts were triggered and we observed that the gel did not constitute a separating barrier and the serum did not occur. We evaluated that the underlying causes of this condition could be multiple myeloma, any radio-contrast dye usage or dialysis catheters. A second sample was collected into BD Vacutainer CAT (Clot Activator Tube) and the serum did not occur, also. A subsequent blood sample was collected into BD Vacutainer Barricor LH Plasma tube which has a mechanical separator and after centrifugation biochemical analyses were performed with plasma.

RESULTS:The analysis resulted a highly increased IgG $(111 \mathrm{~g} / \mathrm{L}$ (reference interval 7.51-15.6 g/L)) and total protein (120.32 g/L (reference interval 66$83 \mathrm{~g} / \mathrm{L})$ ) concentration. With these results a bone marrow examination was performed and the patient was diagnosed with Multiple Myeloma.

CONCLUSIONS:This case report confirms that laboratories and tube manufacturers should be aware of the limitation of the separator gel tubes in patients with high plasma density and its effects on test results.

Keywords: separator gel, blood collection tube, hyperproteinemia

\section{O-019}

Evaluation of inflammatory status with procalcitonin and neopterin in healthy overweight and obese adults based on waist-hip ratio

Murat Cağlayan ${ }^{1}$, Cigdem Sonmez $^{2}$, Nurcihan Dolu ${ }^{3}$, Fatih Özcan ${ }^{3}$, Erdinç Serin ${ }^{3}$, Güler Buğdayc1 ${ }^{4}$

${ }^{1}$ Yıldırım Beyazıt University Yenimahalle Training and Research Hospital, Medical Biochemistry Ministry of Health General Directorate of Emergency Health Services Ankara, Turkey

${ }^{2}$ University of Health Sciences, Dr Abdurrahman Yurtarslan Oncology Training and Research Hospital, Department of Clinical Chemistry, Ankara Turkey ${ }^{3}$ Şişli Etfal Hamidiye Etfal Training and Research Hospital, Medical Biochemistry Laboratory, İstanbul, Turkey ${ }^{4}$ University of İzzet Baysal Department of Biochemistry, Bolu Turkey

OBJECTIVES:In this study we aimed to evaluate the role of hs-CRP, Procalcitonin (PCT) and neopterin as inflammatory markers in the diagnosis of chronic low-grade inflammation associated with obesity.

MATERIALS and METHODS:67 obese, overweight and healthy adults with mean age of $41.1 \pm 10$ years were included in the study. All participants were divided into two groups according to waist hip ratio ( $<0.9$ Group-A, $\geq 0.9$ Group-B) and three groups according to body mass index (BMI) ( $<25$ Group- 1, 25-29 Group$2, \geq 30$ Group-3). Hs-CRP, PCT, neopterin levels of the groups were compared between the groups. Lipid profile and blood glucose levels also evaluated.

RESULTS:There was no significant difference in CRP, NP, PCT between the groups formed according to waist hip ratio ( $\mathrm{p}>0.05)$. In BMI groups, CRP levels were found to be elevated with obesity in BMI groups. There was a difference between Group-1 and Group-2 and Group-2 and Group-3, but they were not significant. The difference between Group 1 and Group 3 was significan $t(p$ $<0.05)$. There was no difference in NP levels between the groups $(\mathrm{p}>0.05)$. In the PCT levels, there were statistically significant results between Group-1 and Group-2 and between Group-1 and Group-3, but no difference was found between Group 2 and Group 3( $\mathrm{p}>0.05)$.

CONCLUSIONS:It was shown that the increase in total fat mass in the body may lead to an increase in inflammation markers. However, it was concluded that this difference may be more closely related to the degree of obesity rather than fat distribution

Keywords: Obesity, Inflammation, Hs-CRP, Procalcitonin, Neopterin

\section{O-021}

Simultaneous determination, quantitation and validation of the most used benzodiazepines in urine

Cigdem Karakukcu, Derya Koçer, Mine Yüce Faydalı Department of Biochemistry, Kayseri City Hospital,Kayseri, Turkey

OBJECTIVES:A single method for confirmation and quantitation of a panel of commonly prescribed benzodiazepines and metabolites, flunitrozepam, clonazepam, diazepam, lorazepam, oxazepam, bromozepam, clobazam, flurazepam, midazolam, and triazolam was developed for urine samples.

MATERIALS and METHODS:Quantitation was by liquid chromatography tandem-mass spectrometry (LC-MS-MS) using a AB-Sciex 4500 Q-TRAP system. The instrument was operated in multiple reaction monitoring mode with an electrospray ionization source in positive ionization mode. Deuterated analogues were included as internal standards for all 10 analytes. The method was evaluated for recovery, bias, imprecision, linearity, analytical range, carryover, and matrix effect.

RESULTS:The measurement of calibration dependence allowed to determine the extent of linearity in the concentration range from 12.5 to $500 \mathrm{ng} / \mathrm{ml}$ for all benzodiazepines except midazolam flunitrozepam, clonazepam, oxazepam, clobazam, which were linear through 25 to $500 \mathrm{ng} / \mathrm{ml}$ with acceptable coefficients of determination (r2>0.99). For all analytes at concentration levels 12.5, 300 and 
$500 \mathrm{ng} / \mathrm{ml}$ ( $\mathrm{n}=5,3$ days) BIAS and precision (within-run and between-run) were established in the range 2.7 to 4.10 and 0.83 to 9.90 , respectively. Detection limits (LOD) ranged at $0.95-7.27 \mathrm{ng} / \mathrm{ml}$ and quantification limits (LOQ) at 2.89$22.04 \mathrm{ng} / \mathrm{ml}$ for all analytes, which were both highest for Clobazam. No carryover was observed after the injection of $500 \mathrm{ppb}$ certificated reference material. For matrix effect evaluation, relative recovery was established between $91 \%$ to $97 \%$, except midazolam (absolute recovery $88 \%$, relative recovery $76 \%$ ).

CONCLUSIONS:The applicability of a simple LC-MS/MS method was proven by for analyzing authentic urine samples and third party external quality samples in urine matrix.

Keywords: Benzodiazepines, Validation, LC-MS/MS method

\section{O-022}

Association of NUCB2/Nesfatin-1 gene polymorphism with obstructive sleep apnea severity

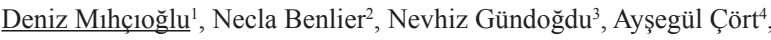
Erman Kandilli

${ }^{1}$ Department of Nutrition and Dietetics, Faculty of Health Science, SANKO

University, Gaziantep, Turkey

${ }^{2}$ Department of Medical Pharmacology, Faculty of Medicine, SANKO

University, Gaziantep, Turkey

${ }^{3}$ Department of Pulmonary Diseases, Faculty of Medicine, SANKO University,

Gaziantep, Turkey

${ }^{4}$ Department of Biochemistry, Faculty of Medicine, Pamukkale University,

Denizli, Turkey

${ }^{5}$ Department of Medical Pharmacology, Faculty of Medicine, Gaziantep

University, Gaziantep, Turkey

OBJECTIVES:Obstructive sleep apnea syndrome (OSAS) is characterized by repetitive obstruction of upper respiratory tract and associated with decrease in blood oxygen saturation. Inflammation is involved in the mechanism of obstructive sleep apnea syndrome. The aim of the study was to determine distribution of NUCB2 genotypes of nesfatin-1 in OSAS.

MATERIALS and METHODS:38 OSAS and 12 healthy subjects were enrolled in this study. Individuals were seperated into four groups (mild, moderate, severe and normal) by standard polysomnography, according to the apnea-hypopnea index. Chronic inflammatory diseases, psychiatric diseases, malignant diseases and neurogenic diseases, were excluded from the study. After sampling, DNA isolation and Real-Time PCR were performed.

RESULTS:The mean age of paticipants were $52.02 \pm 10.40$ years. $\% 18$ of volunteers were male and $\% 82$ of volunteers were female. Control group consist of $12(\% 24)$ individuals had an $\mathrm{AHI}<5$. NUCB2 gene polymorphisms were compared with sleep scores (severe, moderate, mild and control). There was a significant relationship between rs2634462 polymorphism and sleep scores $(\mathrm{p}=0.002)$. There was no relationship between $\mathrm{rs} 1330 \mathrm{~A} / \mathrm{G}, \mathrm{rs} 214101 \mathrm{C} / \mathrm{T}$, rs757081 C/G NUCB2 gene polymorphisms and sleep scores.

CONCLUSIONS:It was found that individuals with homozygous $\mathrm{C} / \mathrm{C}$ genotype of the rs 2634462 polymorphism had significantly more severe OSAS compared to individuals with other genotypes. rs2634462 polymorphism of nesfatin-1 may be a new biomarker in predicting the presence of OSAS.

Keywords: NUCB2 gene, Obstructive Sleep Apnea Syndrome, Polymorphism

\section{O-023}

The effect of lycopene on autophagy in fluoride toxicity in kidney cells

Ayșe Usta $^{1}$, Veysel Yüksek ${ }^{2}$, Sedat Çetin ${ }^{3}$, Semiha Dede ${ }^{3}$

${ }^{1}$ Van Yuzuncu Yil University, Department of Chemistry, Faculty of Science,

Van, Turkey

${ }^{2}$ Van Yuzuncu Yil University, Ozalp Vocational High School, Van, Turkey

${ }^{3}$ Van Yuzuncu Yil University, Department of Biochemistry, Faculty of Veterinary Medicine, Van, Turkey

OBJECTIVES:The aim of this study was to investigate the effect of lycopene on autophagic pathway against toxicity induced by sodium fluoride in renal cell line (NRK-52E).

MATERIALS and METHODS:Cells were grown in vitro by regular passages. The IC50 value of $\mathrm{NaF}$ and the proliferative concentration of lycopene were determined by MTT. In the study, 4 groups were formed as control (K), fluorine (F), lycopene (L) and fluorine lycopene (FL). 24 hours after the application of fluorine and lycopene to the cells at the specified concentrations, RNA isolation and cDNA synthesis were performed and expression of the autophagic genes was determined by RT-PCR.

RESULTS:The proliferation enhancing concentration of lycopene $(1 \mu \mathrm{M})$ and the IC 50 concentration of $\mathrm{NaF}(3200 \mu \mathrm{M})$ at $24 \mathrm{~h}$ were found. In the FL and F groups, Sqstm 1 expression increased 19 and 9 times, Atg5, 9 and 5.5 times, Map11c3a 6 and 5 times, respectively. There was no significant change in other genes.

CONCLUSIONS:As a result, it was determined that the fluoride given at IC50 concentration affects the autophagic genes studied and the highest increase occurred in Sqstm 1. It can be concluded that lycopene given alone does not alter the genes much, and in the FL group, fluorine may increase autophagy by inhibiting the proliferative effect of lycopene.

Keywords: fluorine, cell culture, lycopene, autophagy

\section{O-025}

The relationship between WNT signaling activity and organ attitudes in scleroderma disease sub-groups

Ayse Kocak $^{1}$, Duygu Harmanc1 ${ }^{1}$, Gul Guner Akdogan ${ }^{3}$, Merih Birlik ${ }^{2}$

${ }^{1}$ Dokuz Eylul University, Molecular Medicine Faculty, Izmir

${ }^{2}$ Dokuz Eylul University, Medicine Faculty, Rheumatology \& Immunology

Department, Izmir

${ }^{3}$ Izmir University of Economics, Medicine Faculty, Medical Biochemistry, Izmir

OBJECTIVES:Scleroderma is a chronic inflammatory autoimmune disease characterized by fibrosis in the skin and internal organs. The relationship between SCC type,stage,pathogenesis, organ involvement and WNT gene family has not been identified yet.We aimed to show the relationship of WNT gene family and antagonists in development of SSC subtypes of disease and different organ involvement.

MATERIALS and METHODS:The study included 85 patients with SSC and 77 controls. The gene expressions \& protein levels of the WNT family and antagonists were analyzed from blood samples. The qPCR method was used for WNT gene expression levels. WNT antagonists protein levels were determined by ELISA method. The relationship between these parameters and disease stage, type and organ involvement was evaluated.

RESULTS:There was a significant increase in WNT-1, WNT-10b, WNT-2, and WNT-6 genes in the SCC group.Axin-2 is decreased. DKK-1 and Kremen protein expressions are decreased in scleroderma. There was a significant difference between WNT-3a and WNT-10a gene expression among patients with generalized scleroderma and limited SCC.WNT-3a and WNT-10a gene expression increased in generalized scleroderma. WNT-1, WNT-2 and AXIN2 gene expression increased significantly in PAH positive SCC patients. There was a positive correlation between the modified Rodnan skin score (MRS) and WNT-2 in patients with SCC.There was a significant positive correlation between total GIS involvement score and WNT-1, WNT-2, WNT-4, WNT-8a, WNT-9b in scleroderma patients. WNT-1, WNT-2, WNT-4, WNT-8a, WNT-9b gene expression expressions increased as the disease severity scale increased. CONCLUSIONS:WNT-1 and WNT-2 were found to be high in the skin and organ involvement of scleroderma. It was found to play a role in the pathogenesis of the disease.Therefore, we identified new therapeutic targets in SCC. Keywords: WNT signaling pathway, WNT antagonists, scleroderma, organ involvement 
O-027

Towards the clinical implementation of pharmacogenetics in cardiology: Serbian experience

Sanja Stankovic ${ }^{1}$, Milika Asanin ${ }^{2}$, Goran Stankovic ${ }^{2}$

${ }^{1}$ Center for Medical Biochemistry, Clinical Center of Serbia, Belgrade, Serbia; Faculty of Pharmacy, Business Academy University Novi Sad, Serbia

${ }^{2}$ Department of Cardiology, Clinical Center of Serbia, Belgrade, Serbia; Faculty of Medicine, University of Belgrade, Belgrade, Serbia

OBJECTIVES:The use of pharmacogenetic testing in cardiology is rapidly expanding and constantly refining. The objective of this study was to evaluate the effect of the CYP2C19, ABCB1, PON1 and P2RY12 variants on clopidogrel pharmacodynamics and clinical outcomes in Serbian ST-elevation acute myocardial infarction (STEMI) patients undergoing primary PCI (pPCI).

MATERIALS and METHODS:One hundred and forty consecutive patients referred to pPCI for STEMI in a high-volume cath lab were enrolled in the study. Clopidogrel response was assessed with multiple electrode platelet aggregometry. The clopidogrel-metabolizing pathway SNPs used were: CYP2C19*2 (rs4244285), CYP2C19*3 (rs4986893), CYP2C19*17 (rs12248560), ABCB1 (rs1045642), PON1 (rs854560, rs662), and P2RY12 (rs2046934). The primary clinical endpoint was major adverse coronary and cardiovascular event (MACCE) defined as death, nonfatal myocardial infarction, ischemia-driven revascularization and stroke. The secondary clinical endpoint was bleeding occurrence. Bleeding was defined according to the Bleeding Academic Research Consortium definition. The follow-up period was one year.

RESULTS:One-year MACCE was $12.9 \%$. All alleles and genotype proportions were found to be in Hardy-Weinberg equilibrium ( $p>0.05$ ). Among the SNPs tested, only CYP2C19*17 was significantly associated with MACCE, but not with clopidogrel response. Our results did not find CYP2C19*2 or CYP2C19*3 to be significantly associated with MACCE. Bleeding was not significantly different across the CYP2C19, ABCB1, P2RY12 and PON1 genotype groups. CONCLUSIONS:In clopidogrel-treated patients with STEMI undergoing pPCI, CYP2C19*17 was independently associated with an increased risk of MACCE independent of clopidogrel responsiveness. The bleeding risk does not appear to be explained by CYP2C19 genotype.

Keywords: clopidogrel, pharmacogenetics, STEMI

\section{O-030}

Inhibitory effect of glyphosate on butyrylcholinesterase and acetylcholinesterase activity

Ayșe Ulusoy ${ }^{1}$, Kezban Kartlaşmış ${ }^{1}$, Safiye Taga ${ }^{2}$, Nurten Dikmen ${ }^{1}$

${ }^{1}$ Cukurova University Faculty of Medicine, Department of Medical

Biochemistry, Adana

${ }^{2}$ Mersin University Faculty of Medicine, Department of Gynecology and

Obstetric-Center of Assisted Reproduction, Mersin

OBJECTIVES:Herbicide glyphosate (N-phosphonomethyl glycine) began to be used in 1974 for weed control in agricultural production areas. In 2015, the World Health Organization(WHO) classified glyphosate as a possible carcinogen for humans. Increased glyphosate use was correlated with various types of cancer, Alzheimer, autism and Parkinson's diseases. Cholinesterase enzymes are found in large amounts in the brain and also inhibit organophosphate poisoning. Therefore, we aimed to make a preliminary study on the effect of glyphosate on cholinesterase enzyme types.

MATERIALS and METHODS:Inhibition effects of glyphosate at various concentrations $(282 \mathrm{mg} / \mathrm{L}, 28.2 \mathrm{mg} / \mathrm{L}, 2.8 \mathrm{mg} / \mathrm{L}, 0.7 \mathrm{mg} / \mathrm{L})$ of acetylcholinesterase in human erythrocytes and butyrylcholinesterase in human plasma were examined for $10 \mathrm{~min}, 30 \mathrm{~min}$ and 1 hour preincubation periods. Colorimetric kinetic measurements of cholinesterases were performed using the Ellman's method.

RESULTS:The decrease in measured butyrylcholinesterase enzyme activity was measured in the different glyphosate concentrations depend on preincubation periods. The most important decrease was observed at butyrylcholinesterase with $31 \%$ loss of activity at a concentration of $282 \mathrm{mg} / \mathrm{L}$ glyphosate in 1 hour preincubation. Acetylcholinesterase enzyme activity decreased $11 \%$ activation at a high concentration of glyphosate $282 \mathrm{mg} / \mathrm{L}$, while a decrease in timedependent on preincubations was not measured. There was no decrease in acetylcholinesterase activity at other glyphosate concentrations.
CONCLUSIONS:Some articles have controversial statements about the inhibition of glyphosate on cholinesterases. Because of the effect of glyphosate in neurological diseases, we investigated the interaction of both enzymes with glyphosate in vitro conditions. It was concluded that prolonged exposure to glyphosate may cause pathological findings.

Keywords: Glyphosate, Acetylcholinesterase, Butyrylcholinesterase, Ellman's Method

\section{O-031 \\ Evaluation of Roche Accu-Chek Inform II Glucose test strip system in the hospital setting}

\section{Settar Kosova}

Çaycuma/Zonguldak State Hospital, Zonguldak, Turkey

OBJECTIVES:Glucometers are widely used in hospital wards as a practical tool to get immediate results concerning the patient's glucose status. Accurate bedside glucose measurements are of paramount importance in the evaluation and treatment of diabetic patients.

MATERIALS and METHODS:According to our institutional quality assurance policy, weekly venous blood samples are analyzed on the sampling by Glucometer (Roche Accu-Chek Inform II, performed by nurses) and the Laboratory (Roche Cobas c6000, c501). 246 patients' venous blood glucose results (Glucometer and Laboratory) were analyzed. Wilcoxon test was performed on paired venous blood sample results. Performance according to ISO 151972013 standard including consensus error grid analysis was investigated.

RESULTS:Glucometer and Laboratory median glucose $(\mathrm{mg} / \mathrm{dl})$ levels were 136,5 (range: $75-461)$ and 134,5 (range: $71-441)$ respectively $(\mathrm{p}=0,0061$, Wilcoxon). Coefficient of variation of paired results was 7,7 \% (95 \% CI: 5,9 $9,2) .91,1 \%$ of glucometer glucose results $(224 / 246)$ were within allowed limits according to ISO 15197 2013. This performance did not meet the ISO 15197 2013 standard which requires that at least $95 \%$ of results should be within limits. Nevertheless, all 22 patients glucose data beyond the standard's limits were in the zones of a ( $86 \%)$ and $b(14 \%)$ of consensus error grid area. Thus fulfilling standard's 2 nd necessity at a rate of $100 \%$ (at least $99 \%$ required)

CONCLUSIONS:According to our routine quality control performance data, the Roche Accu-Chek Inform II glucometer system is clinically useful for professional use in health care institutions.

Keywords: venous glucose, Accu-chek inform II, Cobas 6000, ISO 15197 2013, consensus error grid

\section{O-032}

Evaluation of urine drug screening test results between 2016-2018 years in Kanuni Education and Research Hospital Laboratory

Nazime Cebi, Hüseyin Yılmaz, Nizar Türker, Neslihan Kayaoğlu, Birsel Yayla University of Health Sciences, Kanuni Education and Research Hospital Laboratory, Trabzon, Turkey

OBJECTIVES:Drug abuse one of the most important health problems in the world and unfortunately it is rapidly increasing in Turkey as well. In order to establish valid policies on this issue, the initial step is to define the extent of this problem by determining the prevalence of use. Further information on the frequency of substance use is essential for preventive studies. We planned this study to determine which drugs are analyzed more widely in Trabzon and to evaluate their distribution according to age and sex, and therefore to collect and present data to take measures.

MATERIALS and METHODS:Urine drug screening tests (Amphetamine, Benzodiazepine, MDMA-Ectacy, Barbiturate, THC-Cannabis, Cocaine, BonzaiSpice1 / Spice2, Opiate, Buprenorphine) were evaluated with retrospective LIS data and the results were determined as positive by age and sex.

RESULTS:Cannabis (THC) was commonly used banned substance in $16 \%$ of patients admitted between 2016-2018, followed Benzodiazepine (9.97\%) and Buprenofrin $(8.93 \%)$. Bonzai spice1 and spice2, which are thought to be widely used, are $0.045 \%$ and $0.3 \%$ respectively. The important reason for the low usage of this substance, which has increased in recent years, is the existence of product variety which limits its detection by the current method. Percentages of other substances were cocaine $0.3 \%$, MDMA $1.76 \%$, Opiate $1.37 \%$, Amphetamine 
$2.25 \%$, Barbiturate $0.058 \%$, respectively. $95.61 \%$ of the user is male, $4.51 \%$ is female and the average age is around 31 years and the lowest-highest age is 14-

CONCLUSIONS:The increase in the frequency of substance use among women and decreasing user age raises concern. However, the research is regional and countrywide studies are needed.

Keywords: drug abuse

\section{O-033}

\section{Pregabalin substance abuse}

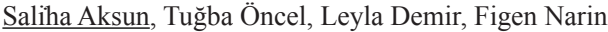

Department of Medical Biochemistry, Izmir Katip Celebi University Faculty of Medicine, Izmir,Turkey

OBJECTIVES:Pregabalin is an antiepileptic drug that reduces the release of excitatory neurotransmitters such as glutamate, substance P. It is approved to be used in adult patients who have peripheral neuropathic pain, fibromyalgia, epilepsy. Drug bind to the $\alpha 2-\delta$ subunit of voltage-dependent calcium channels in central nervous system. It is well known that pregabalin has some abuses potentially. Several databases have warned for overdose fatalities. Overdoses of gabapentinoids can become lethal in mixture with other psychoactive drugs, especially opioids.In this study, we aimed to reveal the abuse of pregabalin.

MATERIALS and METHODS:In our laboratory, drug analysis is carried out by mass spectrometry method with QTrap analyzer. These analysis were performed on urine samples. All results which analysed between 01.02.2019-10.07.2019 were examined retrospectively. Pregabalin positive samples were evaluated among the results. Amphetamine and its derivatives, opioids, codeine, morphine, heroin, cannabis, cocaine, benzodiazephines, synthetic cannabinoids, pregabalin, gabapentin analyzed in all samples.

RESULTS:1522 patients results evaluated. In 22.8\% (347/1552) samples, pregabalin concentration was higher then $50 \mathrm{ng} / \mathrm{ml}$. It has been found that 140 positive pregabalin results have a level of over $1000 \mathrm{ng} / \mathrm{ml}$. Moreover, cannabis has been found on 68 samples in addition to pregabalin. In some samples it has also been found amphetamine or cocaine and multiple substance were positive with pregabalin

CONCLUSIONS:According to our data pregabalin abuse is common. Since pregabalin can cause undesirable consequences like other addictive substances, health professionals and prescribers must be aware of this misuse potential. Laboratory professionals should be able to measure pregabalin in drug laboratories.

Keywords: Pregabalin, Lyrica, substance abuse

\section{O-034}

\section{The protein supplements and inhibition of liver enzymes at athletes}

\section{Nafija Serdarevic}

Institute for Clinical Biochemistry and Immunology University of Sarajevo Clinics Center, Faculty of Health Sciences, University of Sarajevo, Bosnia and Herzegovina

OBJECTIVES:The aim of study was to investigate influence of protein supplements changes on liver enzymes (ALT, AST, y -GT and LD) in athletes with high and low intensity training.

MATERIALS and METHODS:The 180 male athletes were divided in three groups of subjects, athletes with high intensity training, athletes with low intensity training and the control group. We analyzed the activity of enzymes ALT, AST, $\mathrm{y}$-GT and LD, proteins in the urine, as well as urea and creatinine. The enzyme activity was determinated with a BS-200 Mindray machine.

RESULTS:The results of enzymes activity in vitro show that using protein supplements increase activity of these enzymes: ALT $56.68 \%$, AST $48.78 \%$, $\mathrm{X}$-GT 14.17 and LD $9.71 \%$ in the serum of athletes during training comparing the athletes who do not use supplements. The mean differences between the parameters ALT, AST, y -GT and LD between the groups was a statistically significant $(p<0.05)$ between subjects who use supplements and those who do not use supplements. The Man Whitney $U$ test showed that between subjects (high intensity and low intensity training) there is a statistically significant difference between the all examined parameters, while the LD did not show a statistically significance difference $(\mathrm{p}>0.05)$

CONCLUSIONS:The protein supplements (Whey protein, Gainer, Isoactive, BCAA) increased activity of the enzyme ALT, AST, y -GT, LD in athletes. The activity of the enzyme decreases in the serum after a seven-day break of using the protein supplements

Keywords: liver enzymes, Whey protein, Gainer, Isoactive, BCAA

\section{O-035}

Effect of bariatric surgery on ghrelin-hepatosteatosis interaction:

The Selcuk University Faculty of Medicine example

Meryem Ayranci ${ }^{1}$, Hakan Vatansev ${ }^{2}$, Husamettin Vatansev ${ }^{3}$, Huseyin Yilmaz ${ }^{1}$ Department of Nutrition and Dietetics, Faculty of Health Sciences, Necmettin Erbakan University, Konya, Turkey

${ }^{2}$ Department of Food Processing, Meram Vocational High School, Necmettin Erbakan University, Konya, Turkey

${ }^{3}$ Department of Clinical Biochemistry, Faculty of Medicine, Selcuk University, Konya, Turkey

${ }^{4}$ Department of General Surgery, Faculty of Medicine, Selcuk University, Konya, Turkey

OBJECTIVES:During the last years, bariatric surgery has become an established procedure for effective and sustainable weight loss. In the majority of patients, bariatric surgery improves liver steatosis, inflammation, and fibrosis in nonalcoholic fatty liver disease patients with obesity. The aim of our study was to investigate the effect of bariatric surgery on ghrelin-hepatosteatosis interaction in morbid obese patients.

MATERIALS and METHODS:23 patients who underwent bariatric surgery $(\mathrm{BMI}=49.27 \pm 7.46 \mathrm{~kg} / \mathrm{m} 2)$ in Selcuk University Faculty of Medicine Clinic of General Surgery were included in the study. Sixteen of these patients were operated with laparoscopic sleeve gastrectomy and seven were operated laparoscopic Roux-en-Y gastric bypass method. Blood samples were collected from the patients before of the operation and at 1st, 3rd, 6th months after the operation. Hepatosteatosis were performed by radiologists with ultrasonography. Ghrelin levels were studied by elisa method.

RESULTS:There was not found significant difference in ghrelin levels between preoperative and postoperative periods $(p=0.384)$. The hepatosteatosis was significantly decreased at postop 1st, 3rd, 6th months compared to preop period $(\mathrm{p}<0.05)$. There was a weak, significant and negative correlations between ghrelin levels and hepatosteatosis at postoperative 1st and 3rd months.

CONCLUSIONS:As a result, bariatric surgery has improved some endocrine abnormalities, but did not show any significant difference in ghrelin levels. Significant reductions in hepatosteatosis were observed after surgery. Further studies involving ghrelin and hepatosteatosis should be perform.

Keywords: Bariatric surgery, ghrelin, hepatosteatosis

\section{O-036}

The results in two different provinces in Black Sea Region where thalassemia screening was implemented:a rare hemoglobin variant

\section{Durmus Ayan}

Department of Medical Biochemistry, Amasya Public Health Laboratory and Department of Medical Biochemistry, Amasya University Sabuncuoglu Serefeddin Research and Training Hospital, Amasya, Turkey

OBJECTIVES:We aimed for assessing the results of a thalassemia test implemented for the purpose of screening in the provinces of Amasya and Tokat and for revealing the clinical features of a rare variant type of hemoglobin in our study.

MATERIALS and METHODS:The results of $n=2258$ samples ( $55.8 \%$ males and $44.2 \%$ ), sent from the provinces of Amasya and Tokat for the purpose of thalassemia(hemoglobin variant) screening between 15.10.2018-31.05.2019 were retrospectively examined in Public Health Laboratory in Central Amasya. Hemoglobin variant analysis was carried out through the method of HPLC (High Performance Liquid Chromatography) on Primus Ultra2 device. The sample was also examined on a different system for the rare hemoglobin variant (Hb Pusan) and DNA strand analysis was implemented for substantiation for $\mathrm{Hb}$ Pusan variant. 
RESULTS:In accordance with the results regarding patients retrospectively screened, while the results of $\mathrm{n}=2170$ ( $56.3 \%$ males and $43.7 \%$ females), patients were found to be normal; 37 patients (40.6\% male and $\% 59.4$ female) with suspected of alpha thalassemia was detected), 50 patients (44\% males, $56 \%$ females) with suspected of beta thalassemia was detected, hemoglobin 1 female patient with suspected of hemoglobin E variant was detected and hemoglobin Pusan variant was detected in $n=1$ male patient.

CONCLUSIONS:In accordance with our findings, it was discovered that frequency of beta thalassemia is higher than other types of variant in both provinces.

Keywords: thalassemia, hemoglobin variant analysis, HPLC, hemoglobin Pusan

\section{O-037}

First observation of Hemoglobin Hamilton [ß11(A8)Val $\rightarrow$ Ile] in Turkey

$\underline{\text { Irem Yildiz }}{ }^{1}$, Diclehan Oral ${ }^{2}$, Selahattin $\mathrm{Keleş}^{2}$, Mehmet Akif Çürük

${ }^{1}$ Department of Biochemistry, Institute of Health Science, Cukurova University, Adana, Turkey

${ }^{2}$ Department of Genetic, Medical Faculty, Dicle University, Diyarbakır, Turkey

${ }^{3}$ Department of Biochemistry, Medical Faculty, Cukurova University, Adana, Turkey

OBJECTIVES:Until today, approximately 60 hemoglobin variants have been identified in Turkey. One of them, Hb Hamilton, $\beta 11(\mathrm{~A} 8) \mathrm{Val} \rightarrow \mathrm{Ile}$, which is a silent mutation, is the substitution of isoleucine for valine in the 11th position of the beta chain. This mutation does not change the function of the hemoglobin molecule. TheUntil today, approximately 60 hemoglobin variants have been identified in Turkey. One of them, Hb Hamilton, $\alpha 2 \beta 211(\mathrm{~A} 8) \mathrm{Val} \rightarrow \mathrm{Ile}$, which is a silent mutation, is the substitution of isoleucine for valine in the 11th position of the beta chain. This mutation does not change the function of the hemoglobin molecule. The variant can not be determined by cellulose acetate, starch or agar gel electrophoresis. It was discovered in an Austrian family living in Canada by Triton X-100 acid-urea polyacrylamid gel electrophoresis in 1984. Then, cord blood samples of 4581 babies born between 1985 and 1986 in Sardinia hospitals screened for $\mathrm{Hb}$ Hamilton using the same method. In this case study we aimed to report the first observation of $\mathrm{Hb}$ Hamilton in Turkey. variant cannot be determined by cellulose acetate, starch or agar gel electrophoresis. It was discovered in an Austrian family living in Canada by Triton X-100 acidurea polyacrylamid gel electrophoresis in 1984. Then, cord blood samples of 4581 babies born between 1985 and 1986 in Sardinia hospitals screened for $\mathrm{Hb}$ Hamilton using the same method.

MATERIALS and METHODS:Five milliliters of blood samples from patients were collected in ethylenediaminetetraacetic acid (EDTA) vacutainers for estimation of blood count. Hemoglobin variants were characterized by high performance liquid chromatography (HPLC). Micro column method was used for the isolation of DNA samples. Screening of beta globin gene was performed by DNA sequence analysis.

RESULTS:During genetic screening of hemoglobinopathies in Diyarbakır, hemoglobin Hamilton was detected by DNA sequence analysis. Fourteen different $\beta$-thalassemia mutations and 3 abnormal hemoglobins (HbS, HbDPunjab, $\mathrm{Hb}$ Hamilton) were detected in 53 adults. Hb Hamilton was seen in combination with beta-thalassemia mutation (IVS1-110 G>A).

CONCLUSIONS:The presence of Hemoglobin Hamilton was reported for the first time in Turkey. This variant could not be detected when the sample was screened by HPLC.

This project was supported by Dicle University Research Project Unit

(TIP.18.001\& TIP.19.008).

Keywords: Hb Hamilton, HbS, HbD-Punjab, $\beta$-thalassemia

\section{O-038}

Glanzmann thrombasthenia: A case report

Aylin Hakligor ${ }^{1}$, Cigdem Sönmez $^{2}$, Fatma Taneli ${ }^{3}$

${ }^{1}$ Adana City, Training and Research Hospital Central Laboratory, Adana, Turkey ${ }^{2}$ University of Health Sciences, Dr Abdurrahman Yurtarslan Oncology Training and Research Hospital, Department of Clinical Chemistry Ankara, Turkeey ${ }^{3}$ University of Celal Bayar,Manisa, Department of Biochemistry

OBJECTIVES:Glanzmann's Thrombasthenia (GT) is a rare autosomal recessive disorder that affects the platelet glycoprotein IIb/IIIa (GPIIb/IIIa) complex and characterized by prolonged bleeding time. The medical history of the patient and the family history of consanguinity crucial while evaluating the patient. In most cases, bleeding symptoms apparent rapidly early in life, but diagnose of GT needs highly specialized centers. In bleeding disorders, routine coagulation tests may be normal so as differential diagnosis specific test such as platelet function and flow cytometry could be used.

MATERIALS and METHODS:A 40-year-old man, presented to the hematology department with spontaneous ecchymosis, was referred to our laboratory for investigating the bleeding disorder. In addition to routine hematological tests, platelet function tests were performed on an Aggram(Helena) agrometer and Innovance-PFA200(Siemens). A Flowcytometeric analysisis (Navios-Ex, Beckman Coulter) was used to confirm the results.

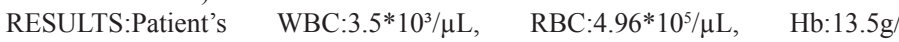
dL, Hematocrit:40.1\%, Platelet: $160 * 10^{3} / \mu \mathrm{L}, \quad \mathrm{PT}: 12.5 \mathrm{sec}, \quad$ aPTT:23sec, Fibrinogen:235mg/dL. Peripheral blood smear was normal. Closure times in PFA analysis; Collagen-epinephrine $>300 \mathrm{sec}$ and Collagen-ADP: $>273 \mathrm{sec}$. In aggregometry, collagen, ADP, epinephrine results were normal, while abnormal aggregation curve with ristocetin was observed. CD42a expression was normal and CD41\&CD61 were reduced in flow cytometry. The patient was diagnosed with GT.

CONCLUSIONS:GT is a rare bleeding disorder. Clinical findings may vary from petechiae, gingival bleeding to severe life-threatening bleeding. Typical characteristics are long bleeding time, normal platelet counts and no peripheral blood smears. When evaluating a patient with bleeding disorder, GT should be kept in mind and further investigations should be performed.

Keywords: Glanzmann Thrombasthenia, Platelet glycoprotein IIb/IIIa, Inherited platelet disorder, Platelet function test

\section{O-040}

Determination of electrochemical behaviour of glucose-6-phosphate dehydrogenase by biosensor

Bașak Günașt1 $^{1}$, Umut Kökbaş ${ }^{1}$, Mustafa Muhlis Alparslan ${ }^{1}$, Kezban Kartlaşmış ${ }^{1}$, Ümmühan Fulden Bozkaya ${ }^{1}$, Güray Kılınççeker ${ }^{2}$, Abdullah Tuli ${ }^{1}$

${ }^{1}$ Department of Medical Biochemistry, Çukurova University, Adana, Turkey ${ }^{2}$ Department of Physical Chemistry, Çukurova University, Adana, Turkey

OBJECTIVES:Glucose-6-phosphate dehydrogenase (G6PD) has a housekeeping role in all cells and is particularly critical to the integrity and functioning of red blood cells. In this study, the activity of G6PD on bioactive surface was investigated. For this purpose, potentiodynamic polarization curves and cyclic voltammetry measurements were used. The surface morphology of the biosensor was investigated by scanning electron microscopy (SEM).

MATERIALS and METHODS:BSA/gelatin and gluteraldehyde was used as a polymer and cross-linking agent, respectively. Cyclic voltammetry measurements were performed using 3-electrode sensing system in $5 \mathrm{mM}$ pH 7.0 phosphate buffer. Gold electrode as working electrode, $\mathrm{Ag} / \mathrm{AgCl}$ as reference electrode, platinum as counter electrode was used. Furthermore, SEM images of enzyme immobilized and enzyme free polymer on electrode surface were compared. RESULTS:Our study showed that G6PD enzyme carried out the oxidation reaction with $2.5 \mu \mathrm{A}$ lower energy. In addition, current flowing reduced by enzyme showed that it complied with the OHM law. From the results obtained G6PD, electrochemical reaction on bioactive surface was found to be effective by reducing energy requirements. Parallel with this information, SEM images also showed surface differences of the enzyme immobilized and enzyme free polymer.

CONCLUSIONS:These potentiometric measurements showed how much enzyme reduced activation energy. Since the main function of enzymes was to 
lower the activation energy, here we have confirmed that the enzyme worked and therefore the immobilization application was successful. Thus, we have completed the preliminary study of a sensitive method that we planned. In further studies, the responses of G6PD enzyme with natural substrates will be evaluated. Keywords: Biosensor, G6PD Enzyme, Immobilization, Polarization, SEM.

\section{O-041}

Investigation of the effect of glyphosate on G6PD activity in in vitro conditions

Kezban Kartlașmiș $^{1}$, Ayşe Ulusoy ${ }^{1}$, Hülya Leventerler ${ }^{2}$, Nurten Dikmen ${ }^{1}$ ${ }^{1}$ Cukurova University Faculty of Medicine, Department of Medical Biochemistry, Adana, TURKEY

${ }^{2}$ Cukurova University Faculty of Medicine, Department of Gynecology and Obstetric-Center of Assisted Reproduction Adana, TURKEY

OBJECTIVES:Glucose 6-phosphate dehydrogenase (G6PD) is a key and rate limiting enzyme in the pentose phosphate pathway. Many substances, especially herbicides, can inhibit G6PD enzyme activity in vitro and in vivo. Glyphosate is the most widely used herbicide in worldwide. The aim of this study was to investigate the effect of glyphosate on the erythrocyte G6PD enzyme in vitro due to the structural similarity of the substrate to the phosphate group of Glucose- 6 phosphate.

MATERIALS and METHODS:In this study, the effect of different glyphosate concentrations $(282 \mathrm{mg} / \mathrm{L}, 28.2 \mathrm{mg} / \mathrm{L}, 2.8 \mathrm{mg} / \mathrm{L}, 0.7 \mathrm{mg} / \mathrm{L})$ on G6PD enzyme activity was investigated. Hemolysate was prepared from erythrocytes obtained from healthy, adult male individuals as samples. Enzyme activity was measured using the Beutler method.

RESULTS:Inhibition percentages for glyphosate administration at different concentrations with $0 / 10 / 30$. minute incubation; $0.1 \mathrm{M}$ glyphosate $21 \%, 24.8 \%$ and $26 \%$ respectively, $0.01 \mathrm{M}$ glyphosate $20.8 \%, 22 \%$ and $11.8 \%, 0.001 \mathrm{M}$ glyphosate $1.5 \%, 9.78 \%$ and $10 \%, 0.0005 \mathrm{M}$ glyphosate $1.3 \%, 6.25 \%$ and $8 \%$, 0.00025 respectively $\mathrm{M}$ glyphosate was observed as $0.07 \%, 2.8 \%$ and $2.14 \%$. The increase in activity observed in the 60 minute incubation suggests that the enzyme is a semi-competitive inhibitor.

CONCLUSIONS:According to the results of our studies, it is seen that inhibition increases due to the increase in the glyphosate concentration and incubation time. Due to the high number of individuals with G6PD enzyme deficiency and increased exposure to glyphosate in the Çukurova region, our study is very important in terms of preventing health problems that may occur.

Keywords: Glyphosate, G6PD, enzyme inhibition

\section{O-042}

The evaluation of microtubes' compatibility to automated process for complete blood count

Fatma Demet Arslan ${ }^{1}, \underline{\text { Ahmet Erkin Bozdemir }}^{1}$, Banu Isbilen Basok ${ }^{1}$, Sukran Copur ${ }^{2}$, Nisel Ozkalay Yilmaz ${ }^{2}$, Harun Akar $^{3}$

${ }^{1}$ Department of Medical Biochemistry, Health Sciences University Tepecik Training and Research Hospital, Izmir, Turkey

${ }^{2}$ Department of Medical Microbiology, Health Sciences University Tepecik Training and Research Hospital, Izmir, Turkey

${ }^{3}$ Clinic of Internal Medicine, Health Sciences University Tepecik Training and Research Hospital, Izmir, Turkey

OBJECTIVES:Newborns and occasionally patients with malignancy develop anemia due to iatrogenic blood loss based on phlebotomy for blood tests, including complete blood count (CBC). Reducing the need for blood transfusion and thus avoiding the associated risks due to frequent phlebotomy involves limiting blood sampling and the use of micro tubes as primary tubes in automated analyzers. It is aimed to compare microtubes with the vacuum tubes of the same brand in terms of accuracy and ease of use.

MATERIALS and METHODS:Venous blood samples were taken from 40 inpatients and collected in three different brand microtubes/evacuated tubes pairs (Microtainer MAP-0.5 mL/Vacutainer-2.0 mL;Becton, Dickinson and Company,USA)(Microvette-0.5 mL/S-Monovette-2.6 mL;Sarstedt Ag \& Co. KG,Germany)(MiniCollect Complete- $0.5 \mathrm{~mL} /$ Vacuette- $2.0 \mathrm{~mL}$;Greiner Bio-One $\mathrm{GmbH}$,Austria). All tubes contained K2EDTA except Microvette. White blood cell(WBC), red blood cell(RBC), hemoglobin, platelet(PLT) were analyzed using a CBC analyzer (DxH 800, Beckman Coulter Inc., USA).

RESULTS:The bias (\%) of WBC, RBC, hemoglobin, and PLT parameters between a microtube and a standard tube for each brand was calculated and presented as follows: Microtainer MAP vs. Vacutainer $-0.41,0.52,0.46$, and -1.34; Microvette vs. S-Monovette -1.09, 0.61, 0.51, and -1.49; MiniCollect Complete vs. and Vacuette, $-0.24,0.34,0.05$, and -0.89 . All bias calculations were within the desirable limits based on the Ricos' biological variation data. CONCLUSIONS:According to the results, laboratories using these CBC tubes can limit blood sampling in their efforts to reduce iatrogenic blood loss, by providing and using micro-volume pairs of the same brand without extra effort, especially in patients requiring frequent phlebotomy.

Keywords: blood cell count, blood specimen collection, laboratory automation, blood volume

O-043

Design of a new biosensor for the determination of ferric iron in blood

Ahmet Ilhan $^{1}$, Umut Kokbas ${ }^{1}$, Abdullah Tul1 ${ }^{1}$, Levent Kayrın

${ }^{1}$ Medical Biochemistry Department, University of Cukurova, Adana, Turkey

${ }^{2}$ Medical Biochemistry Department, University of Kyrenia, Kyrenia, Cyprus

OBJECTIVES:Iron is an element that is necessary for life but can damage the organism if it is present in excess. Iron performs many important functions in the body. Iron deficiency is the most common nutritional deficiency and the leading cause of anemia in the world. In this study, we aimed to design a biosensor for the quantitative determination of $\mathrm{Fe}^{3}+$ in a short time and at an affordable cost. MATERIALS and METHODS:The bioactive layer was prepared by immobilizing hydrogen peroxidase enzymes on the gold electrode with bovin serum albümin (BSA), gelatin, glutaraldehyde with the help of UV light. To ensure separation of ferric iron $\left(\mathrm{Fe}^{3}+\right)$ in the serum from the transferrin, acetate buffer having a $\mathrm{pH}$ of 5.0 was preferred. Hydroxylamine hydrochloride was then used to reduce the ferric iron $\left(\mathrm{Fe}^{3}+\right)$ to ferrous iron $\left(\mathrm{Fe}^{2}+\right)$. Ferrous iron $\left(\mathrm{Fe}^{2}+\right)$ which is produced as a result of the reduction was measured by the reaction of hydrogen peroxidase enzyme.

RESULTS:The response current in the range of 0.2 and $1.4 \mathrm{~V}$ was performed on a cyclic voltammogram at a scanning rate of $0.06 \mathrm{~V} / \mathrm{s}$. Enzymatic reaction rate decreased as substrate concentration increased in an environment where all parameters were constant.

CONCLUSIONS:In determining optimum operating conditions, acetate buffer was determined at $\mathrm{pH} 5.0$ and $200 \mathrm{mM}$ concentration, scanning speed was 0.06 $\mathrm{V} / \mathrm{s}$ and temperature was determined as $40^{\circ} \mathrm{C}$. In this study, the best measurement was obtained with gold electrode immobilized with an enzyme concentration of $0.5 \mathrm{mg} / \mathrm{ml}$ was used.

Keywords: biosensor, hydrogen peroxidase, ferric iron

\section{O-044}

Correlation between LUC \% and thyroid function tests

Arzu Kösem

Ankara City Hospital, Ankara, Turkey

OBJECTIVES:In this study, we aimed to determine the correlation between thirteen hemogram parameters and thyroid stimulating hormone (TSH), thyroxine (T4), and triiodothyronine (T3).

MATERIALS and METHODS:A retrospective study was performed on 18013 patients' data who all presented with thyroid pathologies. Laboratory results of TSH, T4 and T3 blood levels, white blood cells (WBCs), large unprotected cells (LUC), LUC\%, neutrophil count, neutrophil percentage, lymphocyte count, lymphocyte percentage, monocyte count, monocyte percentage, eosinophil count, eosinophil percentage, basophil count, basophil percentage were analyzed. Blood samples were collected by venepuncture and anticoagulated with dipotassium ethylenediamine tetra-acetic acid (EDTA) and hematology parameters were measured within $1 \mathrm{~h}$ of collection on a Siemens ADVIA 2120i hematology analyzer (Siemens Healthcare Diagnostics, Germany). Thyroid function tests were measured by using the ADVIA Centaur XP analyzer (Siemens Healthcare Diagnostics, Germany). Normality tests were performed using single-sample Kolmogorov-Smirnov test using SPSS 18.0 for all data. Correlation analysis was performed using the Pearson method. 
RESULTS:The relationship between TSH, free T3, free T4 and LUC \% were $\mathrm{r}=-0.102, \mathrm{p}=0.001 ; \mathrm{r}=0.210, \mathrm{p}<0.001 ; \mathrm{r}=0.127, \mathrm{p}<0.001$; respectively. CONCLUSIONS:It can be proposed that LUC\% count is weakly but significantly associated with thyroid hormon levels observed.

Keywords: inflammatuary marker; LUC\%; thyroid function tests

\section{O-045}

The effect of Rhamnetine against to ischemia-reperfusion injury in the kidney

\section{Mustafa Nisari}

Department of Nutrition and Dietetics, Faculty of Health Sciences, University of Nuh Naci Yazgan, Kayseri, Türkiye

OBJECTIVES:The purpose of this study was to investigate the possible protective effect of Rhamnetin, as a potent antioxidant on I/R-induced renal injury in rats.

MATERIALS and METHODS:We used 28 male wistar albino rat weight 200-250 $\mathrm{g}$ in this research. The animals were randomly divided into 4 groups. Each experimental group was consisted of seven animals. Rats were subjected to $45 \mathrm{~min}$ of renal pedicle occlusion followed by reperfusion. Control Group (C): Ischemia/reperfusion was not performed to animals. Rhamnetin Group (R): $100 \mathrm{mg} / \mathrm{kg}$ Rhamnetin was administered i.p $30 \mathrm{~min}$ prior to ischemia and immediately before the reperfusion period. Ischaemia/Reperfusion Group (I/R): Rats were subjected to $45 \mathrm{~min}$ of renal pedicle occlusion followed by 24 hours reperfusion. Rhamnetin+Ischemia/Reperfusion Group (R+I/R): Rhamnetin (100 $\mathrm{mg} / \mathrm{kg}$ i.p) was administered $30 \mathrm{~min}$ prior to ischemia and immediately before the reperfusion period. Rats were subjected to $45 \mathrm{~min}$ of renal pedicle occlusion followed by 24 hours reperfusion.

RESULTS:MDA levels were found to be significantly increased whereas SOD and GST enzyme activities were found to be significantly decreased in $\mathrm{I} / \mathrm{R}$ $(\mathrm{p}<0.05)$. However, there were no significantly differences in CAT activities and between the $\mathrm{C}$ and I/R groups. While GST activities were significantly elevated in $\mathrm{R}+\mathrm{I} / \mathrm{R}$ group compared to control group, MDA levels were significantly decreased.

CONCLUSIONS:These results show that treatment with Rhamnetin may prevent the kidney damages due to ischaemia result in increasing oxidant stres peroxidation damages further. This study suggests that Rhamnetin may be a effective antioxidant agent.

Keywords: Rat, Rhamnetin, ischemia, reperfusion

\section{0-046}

The protective effect of resveratrol against cyclosporine A-induced oxidative stress and hepatotoxicity

\section{$\underline{\text { Ilknur Bingul }}^{1}$, Vakur Olgac}

${ }^{1}$ Department of Medical Biochemistry, Istanbul Faculty of Medicine, Istanbul University, Istanbul, Turkey

${ }^{2}$ Department of Pathology, Institute of Oncology, Istanbul University, Istanbul, Turkey

OBJECTIVES:The immunosuppressive agent cyclosporine A (CsA) has hepatotoxic potential. Increased reactive oxygen species (ROS) formation is among the causes leading to hepatotoxicity. In this study, we aimed to investigate the effect of resveratrol (RES) treatment on CsA-induced oxidative stress and hepatotoxicity in rats.

MATERIALS and METHODS:Rats were treated with RES (10 mg/kg/ day; i.p.) for 14 days. CsA (25 mg/kg/day; s.c.) was given during the last 7 days together with RES. Determinations of serum alanine aminotransferase (ALT) and aspartate aminotransferase (AST) activities together with hepatic histopathological examinations were performed. ROS, thiobarbituric acid reactive substances (TBARS), advanced oxidation protein products (AOPP), ferric reducing antioxidant power (FRAP), and glutathione (GSH) levels as well as superoxide dismutase (SOD), and glutathione peroxidase (GSH-Px) activities were measured in the liver tissue.

RESULTS:CsA treatment increased hepatic ROS, TBARS, and AOPP levels significantly as compared to the control group. Although hepatic GSH levels and SOD activity did not alter, FRAP level decreased and GSH-Px activity increased significantly in CsA treated rats. CsA also caused degeneration in hepatocytes and sinusoidal spaces. RES treatment ameliorated histopathological changes and decreased hepatic ROS, TBARS and AOPP levels significantly. However, it did not change serum ALT and AST activities as well as hepatic antioxidant parameters in CsA-treated rats.

CONCLUSIONS:RES does seem to have a protective effect on CsA-induced hepatotoxicity by reducing oxidative stress. Considering the immunosuppressive and hepatoprotective efficiency of RES, the combined use of CsA and RES may be useful in hepatic transplantation therapy by reducing hepatotoxicity and increasing the immunosuppressive effect of CsA

Keywords: Cyclosporine, resveratrol, hepatotoxicity, oxidative stress

\section{O-047 \\ Thiol/Disulphide balance and Ischemia-modified albumin levels in female with iron deficiency anemia}

Emre Avci ${ }^{1}$, Alpaslan Karabulut ${ }^{2}$, Gulcin Alp Avci ${ }^{1}$, Cumhur Bilgi ${ }^{1}$ Hitit University, Faculty of Arts and Sciences, Department of Molecular Biology and Genetics, Corum,Turkey

${ }^{2}$ Hitit University, Faculty of Medicine, Department of Internal Medicine, Corum,Turkey

${ }^{3}$ Yuksek İhtisas University, Faculty of Medicine, Department of Medical Biochemistry,Ankara,Turkey

OBJECTIVES:Iron deficiency and harmful effects of iron deficiency anemia; it develops due to deficiencies in oxygen transport to tissues and deficiencies in iron-containing compounds, in particular enzymes. The oxidative free radicals formed during ischemic events increase the level of ischemia-modified albumin (IMA) by making chemical changes in the albumin molecule. The state of thiol/ disulfide plays a vital role in antioxidant process. We aimed to determine the relationship between native thiol, total thiol, disulfide and IMA in female patients with Iron Deficiency Anemia (IDA).

MATERIALS and METHODS:32 female patients diagnosed with IDA and 24 healthy women were joined in our study. Blood samples were taken for complete blood count (CBC), serum iron,total iron binding capacity (TIBC),ferritin and thiol / disulfide homeostasis tests after fasting for at least 8 hours. IMA Abs. levels were determined by a colorimetric method.Total and native thiols and disulfide were analyzed with a novel spectrophotometric method.

RESULTS:We found lower native thiol (-SH)(378.0 $\pm 135.6 \mu \mathrm{mol} / \mathrm{L})$, disulfide $(113.5 \pm 7.6 \mu \mathrm{mol} / \mathrm{L})$, and total thiols $(-\mathrm{SH}+-\mathrm{S}-\mathrm{S}-)(613.0 \pm 125.8 \mu \mathrm{mol} / \mathrm{L})$ in IDA patients compared to healthy controls (respectively $399.5 \pm 158.8,136.7 \pm 63.3$, and $707.5 \pm 119.23 \mu \mathrm{mol} / \mathrm{L})$. IMA Abs. levels $(0.68 \pm 0.11 \mathrm{AU})$ were higher in IDA patients compared to controls $(0.59 \pm 0.15 \mathrm{AU})$. Total thiols levels were positive correlated with both native thiol $(\mathrm{r}=0.326 ; \mathrm{p}=0.033)$ and disulfide $(\mathrm{r}=0.511$; $\mathrm{p}=0.001)$

CONCLUSIONS:Thiols, disulfide and IMA levels increase with the progression of iron deficiency. IDA decreases the antioxidant capacity of erythrocytes and triggers oxidative stress. Therefore, the hypoxic state and oxidant balance resulting from anemia are important in terms of prognosis of the disease Keywords: Thiol/disulfide homeostasis, Oxidative stress, Ischemia-modified albumin, Iron Deficiency Anemia

\section{O-048}

Effect of hibernation on oxidative equilibrium in ground squirrels

Emre Avci, Tulay Pekmez, Safak Bulut, Secil Eren Hitit University, Faculty of Arts and Sciences, Department of Molecular Biology and Genetics, Corum,Turkey

OBJECTIVES:Animals enter the hibernation by providing the necessary conditions to protect themselves from physiologically adverse seasonal conditions. After hibernation, hibernating animals raise their body temperature to $37{ }^{\circ} \mathrm{C}$ within a few minutes and rapidly increase oxygen consumption by 10-20 times. Oxygen scarcity increases the risk of oxidative stress in sensitive tissues in mammalian torpor. The rate of formation of free radicals and their rate of removal are in equilibrium in the organism. The serious imbalance between antioxidant defense mechanism and free radical formation refers to oxidative stress. In our study aimed to determine the level of oxidative stress in 
hibernation different time (before hibernation, during and after) in liver, lung, heart and kidney of Spermophilus xanthomorphius, Spermophilus citellus and Spermophilus taurensis living in different ecological zones in Turkey.

MATERIALS and METHODS:Eighteen animals (S. citellus (6), S. taurensis (6) and S. xanthomorphius (6)) were included in our study. Three different condition (at hibernation, aroused and non-hibernation stage) data were compared. They were sacrificed under anesthesia. Glutathione (GSH), reactive nitrogen oxide species (NOx) and malondialdehyde (MDA) levels were measured spectrophotometrically.

RESULTS:MDA levels during the hybridization were significantly higher in all tissues of the three species, while GSH levels were found to be low. After the hybridization, MDA and GSH levels were increased before and during the hibernation.

CONCLUSIONS:Our data show that an impaired balance exists between oxidative stress and antioxidant systems in most organs and tissues during hibernation

Keywords: Hibernation, Oxidative stress, Ground Squirrel

\section{O-049}

Cellular protection by Phlomis species in $\mathrm{H}_{2} \mathrm{O}_{2}$-induced oxidative stress

Derviș Birim ${ }^{1}$, Pelin Taştan² ${ }^{2}$ Tuğçe Fafal ${ }^{2}$, Bijen Kıvçak ${ }^{2}$, Taner Dağci ${ }^{3}$, Güliz Armagan

${ }^{1}$ Department of Biochemistry, Ege University, Izmir, Turkey

${ }^{2}$ Department of Pharmacognosy, Ege University, Izmir; Turkey

${ }^{3}$ Department of Physiology, Ege University, Izmir, Turkey

OBJECTIVES:Neuroglia-derived chronic inflammation and oxidative stress play central roles in the pathogenesis of neurodegenerative diseases. Thus, increasing evidence indicates that anti-inflammatory activity of plant species and their chemical constituents may protect neurons against various brain disorders. The genus Phlomis is composed of perennial plants in Lamiaceae family which is represented by 46 species from which 30 are endemic in Turkey. The main constituents of Phlomis species are reported to exert pharmacological activities. In this study, we aimed to evaluate the effects of methanol extracts of Phlomis species in $\mathrm{H} 2 \mathrm{O} 2$-induced oxidative stress in a cellular model.

MATERIALS and METHODS:At first, antioxidant activities of methanol extract were evaluated by DPPH and ABTS assays. The effects of the extracts on cell viability were determined by using WST-1 assay. Cells were pre-treated with various concentrations $(1,10$ and $100 \mu \mathrm{g} / \mathrm{ml})$ of extracts for $2 \mathrm{~h}$ and exposed to $\mathrm{H} 2 \mathrm{O} 2$ for $1 \mathrm{~h}$.

RESULTS:Similar to the results obtained by antioxidant assays, methanol extract at $10 \mu \mathrm{g} / \mathrm{ml}$ concentration provided $36.40 \%$ neuroprotection against $\mathrm{H} 2 \mathrm{O} 2-$ induced toxicity.

CONCLUSIONS:Our preliminary results indicate that more research is needed to establish the role of endemic plants as a potential source of neuroprotective agents for further therapeutic approaches.

Keywords: oxidative stress, Phlomis species, neuroprotection

\section{O-050 \\ Neuroprotection by optimized system extracts of Morus nigra L. Fruits in L-DOPA-induced toxicity}

Gizem Kaftan $^{1}$, Halil Koyu², Serdar Demir ${ }^{3}$, Ozlem Yesil Celiktas ${ }^{4}$, Taner Dagci ${ }^{5}$, Mehmet Zeki Haznedaroglu², Guliz Armagan ${ }^{1}$

${ }^{1}$ Department of Biochemistry, Faculty of Pharmacy, Ege University, Izmir, Turkey

${ }^{2}$ Department of Pharmaceutical Botany, Faculty of Pharmacy, Izmir Katip

Celebi University, Izmir, Turkey

${ }^{3}$ Department of Pharmaceutical Botany, Faculty of Pharmacy, Ege University, Izmir, Turkey

${ }^{4}$ Department of Bioengineering, Faculty of Engineering, Ege University, Izmir, Turkey

${ }^{5}$ Department of Physiology, Faculty of Medicine, Ege University, Izmir, Turkey

OBJECTIVES:Morus nigra L. fruits are rich in polyphenols, flavonoids, and anthocyanins responsible for their antioxidant and anti-inflammatory activities. In this study, we aimed to investigate the potential neuroprotection by optimized system extracts of Morus nigra L. fruits in terms of apoptosis related protein expressions.

MATERIALS and METHODS:Extraction of Morus nigra L. fruits was performed with supercritical carbon dioxide, subcritical water and microwave assisted extraction systems as advanced extraction technologies; following conventional methods as orbital shaker and sonification. Obtained extracts in two different concentrations (10 and $100 \mu \mathrm{g} / \mathrm{ml})$ were evaluated for neuroprotection against L-DOPA-induced cytotoxicity in human neuroblastoma cell lines (SH-SY5Y) by using WST-1 assay. The changes in apoptosis-related proteins (Bax, Bcl-2) were investigated by using Western Blotting technique.

RESULTS:Most of the extracts at indicated concentrations were found to protect cells and regulate Bax and Bcl-2 expression levels in L-DOPA-induced toxicity. The maximum protection was observed following subcritical water extraction system at $100 \mu \mathrm{g} / \mathrm{ml}$ which significantly increased cell survival from $51.68 \pm$ $14.19 \%$ (L-DOPA-treated cells) to $161.88 \pm 20.12 \%$ ( $<<0.05)$.

CONCLUSIONS:Chosen extracts/fractions were found to be significantly antiapoptotic. High yields of polyphenols and other antioxidant compounds by advanced extraction techniques may have roles in protecting neuronal cells via regulating Bax and Bcl-2 proteins. Selection of extraction method is a crucial point for plants to achieve therapeutic potential.

Acknowledgements: This study was supported by the TUBITAK (216S839), IKCU BAP and OYP fund supplied by YOK. Novel Fluidic Technologies Laboratory and FABAL (Ege University) are highly appreciated.

Keywords: Neuroprotection, L-DOPA, apoptosis, Morus nigra L. fruits

\section{O-051}

Dynamic thiol-disulphide balance and thioredoxin reductase enyzme levels in patients with chronic kidney disease

Huseyin Erdal ${ }^{1}$, Oguzhan Ozcan ${ }^{2}$, Faruk Turgut ${ }^{3}$, Salim Neselioglu ${ }^{4}$, Ozcan Erel ${ }^{4}$ ${ }^{1}$ Department of Molecular Biochemistry and Genetics, Hatay Mustafa Kemal University, Hatay, Turkey

${ }^{2}$ Department of Medical Biochemistry, Hatay Mustafa Kemal University, Hatay, Turkey

${ }^{3}$ Department of Nephrology, Hatay Mustafa Kemal University, Hatay, Turkey ${ }^{4}$ Department of Medical Biochemistry, Ankara Yildirim Beyazit University, Ankara,Turkey

OBJECTIVES:We aimed to measure the dynamic thiol-disulfide balance and thioredoxin reductase(TrxR) enzyme levels in patients with chronic kidney disease (CKD) and to investigate their roles in disease pathogenesis by comparing them with systemic oxidative stress and inflammation parameters.

MATERIALS and METHODS:Thirty hemodialyses(HD),30 CKD patients(stage3-5) and 30 controls were included in the study. Fasting blood samples were collected. After centrifugation at $1500 \mathrm{~g}$ for $10 \mathrm{~min}$, serum and plasma samples were portioned and stored at $-80^{\circ} \mathrm{C}$.IMA levels were determined by albumin cobalt binding test(ACB).Dynamic thiol-disulfide balance was determined by the colorimetric method developed by Erel et al. Tumor necrosis factor(TNF- $\alpha$ ) and TrxR levels were determined by ELISA

RESULTS:We found that native and total thiol levels of CKD and HD patients were significantly lower than that of the $\operatorname{control} \operatorname{group}(\mathrm{p}=0.001$ for both). However, disulfide levels were significantly higher in the HD group $(p=0.001)$, but there was no significant difference between control and CKD groups $(\mathrm{p}=0.547)$. A notable negative correlation was found between the native and total thiol levels and IMA(r=-0.628;-0.631),BUN ( $\mathrm{r}=-0.747 ;-0.747)$, and creatinine $(\mathrm{r}=-0.732 ;-0.721)$. There was a significant positive correlation between glomerular filtration rate(GFR) and the thiol levels $(\mathrm{r}=0.835 ; 0.824)$. TrxR levels were significantly higher in the patient groups compared to the controls $(\mathrm{p}=0.001)$. TNF- $\alpha$ and CRP levels of the patient groups were significantly higher compared to the controls $(\mathrm{p}=0.001)$.

CONCLUSIONS:Colorimetric measurement of dynamic thiol levels can be used in disease monitoring as a marker because it is easily applicable in routine clinical biochemistry laboratories. The dynamic thiol balance may be involved in the pathogenesis of CKD and is associated with disease severity. This study was supported by Hatay Mustafa Kemal University, Coordinatorship of Scientific Research Projects.

Keywords: Dynamic thiol-disulfide balance, Thioredoxin reductase, Chronic kidney disease, Hemodialysis 
XXVII. Balkan Clinical Laboratory Federation Meeting BCLF 2019

XXX. National Congress of the Turkish Biochemical Society TBS 2019

O-052

Protective role of lycopene in experimental heart ischemia reperfusion model

Özlem Bozkuşs ${ }^{1}$ Büșra Citill ${ }^{2}$, Sevgi Bakarıș ${ }^{3}$, Ergül Belge Kurutaş ${ }^{4}$

${ }^{1}$ Özlem Bozkuş, Department of Medical Biochemistry, Sutcu Imam University, Kahramanmaras, Turkey

${ }^{2}$ Büşra Çitil, Department of Medical Biochemistry, Sutcu Imam University,

Kahramanmaras, Turkey

${ }^{3}$ Sevgi Bakarış, Department of Pathology, Sutcu Imam University,

Kahramanmaras, Turkey

${ }^{4}$ Ergül Belge Kurutaş, Department of Medical Biochemistry Sutcu Imam

University, Kahramanmaras, Turkey

OBJECTIVES:Ischemia refers to the reduction or cessation of blood flow which results in tissue damage and causes insufficient oxygen and nutrition to the tissues. Oxidative stress due to reperfusion after ischemia causes severe functional and structural damage. Free oxygen radicals are responsible for this damage. Lycopene is a pigment of the carotene family, wich is naturally found in vegatables and fruits. To the best of our knowledge, this is the first study, we aimed to investigate the protective role of lycopene in experimental heart ischemia reperfusion (I/R) model.

MATERIALS and METHODS:Male Wistar rats were randomly allocated into three groups $(\mathrm{n}=8$, each) as control (I/R group), Sham and Lycopene (therapy group) groups. One group received lycopene $(50 \mathrm{mg} / \mathrm{kg} /$ day as intraperitoneally) for both single dose before surgery (I/R+lycopene group), while the other was treated intraperitoneally with $0.09 \%$ saline as group $(0.3 \mathrm{~mL} /$ day $)$ (sham group). However, nothing was given to the I/R group. Then; after the venture and surgical procedure applied to the all rats groups, 10 minutes ischemia and 10 minutes reperfusion of the heart was created. At the end of this experimental, activities of catalase (CAT), superoxide dismutase (SOD) and the levels of malondialdehyde (MDA) as oxidative stress biomarkers were measured as spectrophotometric and, also the levels of nitrotyrosine (3-NTx) and nitric oxide (NO) as nitrosative stress biomarkers were measured by ELISA in heart tissues homogenates.

RESULTS:Oxidative/nitrosative stress was confirmed by the significant elevation in MDA, NO, and 3-NTx levels concentrations in I/R group $(\mathrm{p}<0.05)$. Also, CAT and SOD activities in I/R group were significantly lower than lycopene and sham groups $(\mathrm{p}<0.05)$. However, increased CAT and SOD activities and decreased the levels of MDA, NO and 3-NTx were found in lycopene group compared to I/R and sham groups $(\mathrm{p}<0.05)$.

CONCLUSIONS:We thought that lycopene may play the protective the role against heart I/R damage due to its high antioxidant activity.

Keywords: Lycopene, oxidative/nitrosative stress, heart ischemia-reperfusion

\section{O-055 \\ Oxidative status in degenerated painful intervertebral disc samples: variability with respect to duration of symptoms and type of disease}

$\underline{\text { Hatice Kopar }}^{1}$, Kutsal Devrim Seçinti ${ }^{2}$, Süheyla Özyurt ${ }^{1}$, Ergül Belge Kurutas ${ }^{1}$ ${ }^{1}$ Department of Medical Biochemistry, Faculty of Medicine, Sutcu Imam University, Kahramanmaras/Turkey

${ }^{2}$ Deparment of Brain and Nerve Surgery, Faculty of Medicine, Sutcu Imam University, Kahramanmaras/Turkey

OBJECTIVES:Degenerated discs and endplate abnormalities is postulated as a possible source of low back pain. Oxidative stress plays an important the role in various human diseases. This is the first study, we aimed to investigate the levels of oxidative stress biomarkers in disc samples of patients with Modic Changes. MATERIALS and METHODS:Patients (n:15) were separeted as MCI, II, and III types. Of these cases, 3 had complaints for less than 6 months, whereas 3 patients had been suffering from low back pain and leg pain for more than 6 months. Six patients have been diagnosed with subligamentous type and 3 patients had free fragment type of disc degeneration. The activities of catalase (CAT) and superoxide dismutase (SOD), and the levels of malondialdehyde (MDA) in disc samples were determined on spectrophotometer

RESULTS:Oxidative stress was confirmed by the significant elevation MDA levels and decreased of CAT and SOD activities in MCI compared with other MCs $(\mathrm{p}<0.05)$. The highest CAT and SOD activities were found in patients with MCII compared with the other MCs. However, the levels of MDA showed moderate increase in this group $(\mathrm{p}<0.05)$. In addition, the levels of oxidative stress biomarkers in patients with MCIII were slightly higher than the other MCs $(\mathrm{p}<0.05)$.

CONCLUSIONS:Our findings indicated that oxidative stress in patients with MCI may be aggravated as a result of oxidant/antioxidant imbalance and it may cause formation of the lesion in these patients.

Keywords: : Modic Changes, Disc Samples, Oxidative Stress

\section{O-056 \\ The effect of turmeric on GPER1 and oxidative/nitrosative stress biomarkers in cardiac ischemia reperfusion}

Seda Ikikardeș, Sevgi Bakariş, Ergül Belge Kurutaş

Kahramanmaraş Sütçü İmam University, Faculty of Medicine, Kahramanmaraş, Turkey

OBJECTIVES:Extreme oxidative stress induced by reperfusion after ischemia causes functional and structural damages. Free oxygen radicals are mainly considered as responsible for the damage. It has been known that there is a connection between cardiovascular diseases and estrogen. Estrogen is effective on estrogen receptors alpha and beta, and also recently a new estrogen receptor depending on $\mathrm{G}$ protein has been determined (GPER1). It has been revealed in various researches that turmeric has hypoglycemic, anti-inflammatory, antioxidant and lipid reducing effects. In this first study, it was aimed to investigate the effects of turmeric on GPER1 and oxidative/nitrosative stress parameters in heart ischemia reperfusion injury in rats.

MATERIALS and METHODS:The study was carried out with three groups (treatment, sham and control) of eight rats each. Heart ischemia reperfusion injury was formed experimentally in all rats.Turmeric $(50 \mathrm{mg} / \mathrm{kg})$ single dose was given intraperitoneally in the treatment group. Physiological saline $(0,09 \%$ $\mathrm{NaCl}, 0,3 \mathrm{~mL}$ ) single dose was given intraperitoneally in the sham group. No drugs were given in the control group.

RESULTS:Compared to control and treatment groups, antioxidant enzymes (SOD, CAT) decreased and MDA levels increased in the ischemia reperfusion group $(\mathrm{p}<0,05)$. On the other hand, the levels of antioxidant enzymes in the treatment group approached the control group and MDA levels decreased $(\mathrm{p}<0,05)$.

CONCLUSIONS:As a result of this study, it was determined that turmeric has a protective role against heart ischemia reperfusion injury.

Keywords: Turmeric, cardiac ischemia reperfusion injury

O-057

The impact of acupuncture treatment on dynamic thiol-disulphide

homeostasis and ischemia-modified albumin levels to assess

Yasemin Gündüztepe ${ }^{1}$, Setenay Mit ${ }^{3}$, Ersel Geçioğlu³ , Neslihan Gürbüz ${ }^{1}$, Salim Neşelioğlu², Özcan Erel ${ }^{2}$, Cemal Çevik ${ }^{1}$

${ }^{1}$ Dept. of Clinical Biochemistry, Gazi University Faculty of Medicine, Ankara, Turkey

${ }^{2}$ Dept. of Clinical Biochemistry, Yıldırım Beyazıt University, Faculty of Medicine, Ankara,Turkey

${ }^{3}$ Acupuncture Division, Dept. of Biochemistry, Gazi University Faculty of Medicine, Ankara,Turkey

OBJECTIVES:The aim of this study was to investigate the effect of acupuncture on dynamic thiol-disulphide homeostasis and ischemia-modified albumin (IMA) levels as a novel oxidative stress parameter in migraine patients.

MATERIALS and METHODS:The acupuncture treatment consists of 5 sessions with 2 sessions per week. Blood samples have been collected before performing acupuncture, after the 1st and 5th session of the acupuncture. And for the control group blood samples were collected only once. In this study, the dynamic thiol-disulphide homeostasis and IMA levels in the serum samples of migraine patients and healthy individuals was determined using an automated method newly developed by Erel et al.

RESULTS:There were statistically significant differencies \%SS (Disulphide) / total thiol levels patient with pre and post acupuncture groups compared with control group $(\mathrm{P}<0.05)$. However there was no relationship \%SS (Disulphide) / total thiol levels patient with post acupuncture groups compared with pre acupuncture groups $(\mathrm{P}>0.05)$. The average \%SS (Disulphide) / total thiol levels 
was found to be $9,00 \pm 3,27 \mathrm{mmol} / \mathrm{lt}$ in the patient group and $6,98 \pm 2,62 \mathrm{mmol} /$ lt in the control group. The total \%SS (Disulphide) / total thiol levels of patient group were found to be higher than the control group but not statistically. Thiol disulfide balance and IMA levels, which are oxidative stress markers, were increased in migraine patients compared to the control group and this was statistically significant $(\mathrm{p}<0.05)$. We found that acupuncture treatment caused some decrease in thiol disulfide balance but these results were not statistically significant. Ischemia-modified albumin (IMA) were not correlated with attack frequency, pain intensity, or migraine type. Only 5 sessions could be given to these patients. It is possible that if the number of sessions is increased, a meaningful result can be achieved.

CONCLUSIONS:This study evaluated dynamic thiol-disulphide homeostasis and IMA levels in the serum of patients diagnosed with migraine using a novel automated colorimetric method. Because oxidative stress plays an important role in the pathogenesis of many diseases, thiol chemistry has been recognized as increasingly important. We think the effect of acupuncture on dynamic thioldisulphide homeostasis and IMA in migraine patients has revealed that further animal and human studies are necessary.

Keywords: Migraine; Acupuncture; Complementary Therapies, Oxidative stress;

\section{O-058}

\section{Effect of $\mathrm{N}$-acetylcysteine on cisplatin induced apoptosis in rat kidney}

Inayet Gunturk ${ }^{1}$, Seyda Seydel $^{2}$, Fatma Dagli ${ }^{3}$, Arzu Yay $^{4}$

${ }^{1}$ Department of Midwifery, Nigde Omer Halisdemir University, Nigde, Turkey ${ }^{2}$ Department of Healthcare Services, Nigde Omer University, Nigde, Turkey

${ }^{3}$ Department of Chemistry, Cetin Sen Science and Art Center, Kayseri, Turkey

${ }^{4}$ Department of Histology and Embriology, Erciyes University, Kayseri, Turkey

OBJECTIVES:Cisplatin is one of the most potent and widely used chemotherapeutic agents for treatment of a wide variety of solid tumors in clinic. However, due to various side effects such as nephrotoxicity, its efficiency and therapeutic application are limited. Regarding to reduce its side effects, combination therapies of cisplatin with other drugs have been highly considered to reduce toxicity. $\mathrm{N}$-acetylcysteine (NAC), the $\mathrm{N}$-acetyl derivative of the natural amino acid L-cysteine, is a well known antioxidant and anti-inflammatory agent. In the current study it was aimed to investigate the effects of NAC on cisplatin induced apoptosis in rat kidney.

MATERIALS and METHODS:Twenty four male Wistar rats were separated into 4 equal groups: Control, NAC-250, CP (cisplatin), CP+NAC. Rats in the experimental groups were treated with a single dose of cisplatin intraperitoneally (ip) $(10 \mathrm{mg} / \mathrm{kg}$ ) and NAC (ip, $250 \mathrm{mg} / \mathrm{kg}$ ) for 3 consecutive days. At the end of the experiment, nephrotoxicity was confirmed by blood urea nitrogen and creatinine levels and the apoptotic changes were demonstrated by TdT-mediated deoxyuridine triphosphate nick-end labeling (TUNEL) and caspase-3 levels in rat kidneys.

RESULTS:The number of TUNEL-positive cells and caspase-3 levels were significantly increased by cisplatin at day 3 after its injection. Treating the rats with NAC significantly decreased TUNEL-positive cells and caspase-3 levels. CONCLUSIONS:These data suggest that apoptotic cell death are involved, at least in part, in the pathogenesis of cisplatin induced nephrotoxicity, and inhibition of apoptosis appears to play a central role in the beneficial effects of NAC.

Keywords: Cisplatin; Apoptosis; Caspase-3; N-acetylcysteine; Rat
O-060

Thiol-disulfide homeostasis in diabetic microvascular complications

$\underline{\text { Cuma Mertoglu }}^{1}$, Gulsah Siranli ${ }^{1}$, Taha Abdulkadir Coban ${ }^{1}$, Yücel Karakurt ${ }^{2}$, Alevtina Ersoy ${ }^{3}$, Adalet $\mathrm{Ozcicek}^{4}$, Yusuf Arslan ${ }^{5}$, Gamze Gok $^{6}$, Ozcan Erel ${ }^{6}$ ${ }^{1}$ ClinicalBiochemistry, Erzincan University Faculty of Medicine, Erzincan, Turkey

${ }^{2}$ Ophthalmology, Erzincan University Faculty of Medicine, Erzincan, Turkey ${ }^{3}$ Neurology,Erzincan University Faculty of Medicine, Erzincan, Turkey ${ }^{4}$ Internal Medicine,Erzincan UniversityFaculty of Medicine, Erzincan, Turkey ${ }^{5}$ Biostatistics,Erzincan University Faculty of Medicine, Erzincan, Turkey ${ }^{6}$ Clinical Biochemistry, Yıldırım Beyazıt UniversityFaculty of Medicine, Ankara, Turkey

OBJECTIVES:Retinopathy, neuropathy and nephropathy are microvascular complications of diabetes mellitus. In this study, the role of thiol / disulfide was investigated in the development of diabetic microvascular complications.

MATERIALS and METHODS:Individuals $(n=266)$ were divided into five groups; Group 1; who have diabetes without any complications for at least 10 years, group 2; diabetic nephropathy, Group 3; diabetic neuropathy, 4; diabetic retinopathy. The 5 th group consisted of 50 healthy individuals as the control group. Thiol, disulfide, ferroxidase and ischemia modified albümin (IMA) levels were measured.in the serum.

RESULTS:Nativ thiol, total thiol and native thiol / total thiol were found lower in the retinopathy group than the group with at least 10 years diabetes without any complication, the neuropathy group and the control group $(\mathrm{p}<0.001)$. Disulfide / native thiol and disulfide / total thiol levels were found to be higher in the retinopathy group than all the other groups, also the level of disulfide was higher than the control group and neuropathy group $(\mathrm{p}<0.001)$. Ischemia-modified albumin level was found to be higher in the neuropathy and retinopathy groups than all the other groups $(p<0.001)$. Ferroxidase level was found to be lower in the neuropathy and retinopathy groups than the nephropathy group.

CONCLUSIONS:The disruption of thiol disulphide homeostasis favor of disulfide may play a role in the formation of diabetic retinopathy. Also, increased IMA and decreased feroxidase levels may play a role in the development of diabetic retinopathy and neuropathy.

Keywords: Diabetes mellitus, microvascular complications, thiol- disulfide, ischemia modified albumin, ferroxidase 


\section{O-061}

Biological variation in clinical practice: Bridge between laboratorians and clinicians

Humeyra Ozturk Emre ${ }^{1}$, Fatma Hande Karpuzoglu ${ }^{2}$, Cihan Coskun ${ }^{3}$, Ebru Sezer ${ }^{4}$, Ozlem Goruroglu Ozturk ${ }^{5}$, Fatma Ucar ${ }^{6}$, Hikmetcan Cubukcu ${ }^{7}$, Fatma Demet Arslan ${ }^{8}$, Levent Deniz ${ }^{9}$, Mehmet Şenes ${ }^{10}$, Mustafa Serteser ${ }^{11}$, Cevat Yazıc1 ${ }^{12}$, Doğan Yücel ${ }^{10}$, Abdurrahman Coskun ${ }^{11}$

${ }^{1}$ Department of Medical Biochemistry, Necip Fazil City Hospital,

Kahramanmaraş, Turkey.

${ }^{2}$ Department of Medical Biochemistry, Acibadem Labmed, Istanbul, Turkey.

${ }^{3}$ Department of Medical Biochemistry, University of Health Sciences,

Haydarpasa Training and Research Hospital, Istanbul, Turkey.

${ }^{4}$ Department of Medical Biochemistry and Metabolism Laboratory, Faculty of

Medicine, Ege University, Izmir, Turkey.

${ }^{5}$ Department of Biochemistry, Faculty of Medicine, Çukurova University,

Adana, Turkey.

${ }^{6}$ Department of Clinical Biochemistry, Diskapi Yildirim Beyazit Training and

Research Hospital, Ankara, Turkey.

${ }^{7}$ Department of Biochemistry, Maresal Cakmak State Hospital, Erzurum,

Turkey.

${ }^{8}$ Department of Medical Biochemistry, University of Health Sciences, Tepecik Training and Research Hospital, Izmir, Turkey.

${ }^{9}$ Department of Medical Biochemistry, University of Health Sciences, Istanbul Training and Research Hospital, Istanbul, Turkey.

${ }^{10}$ Department of Medical Biochemistry, University of Health Sciences, Ankara Training and Research Hospital, Ankara, Turkey.

${ }^{11}$ Department of Medical Biochemistry, School of Medicine, Acıbadem Mehmet Ali Aydınlar University, Istanbul, Turkey.

${ }^{12}$ Department of Medical Biochemistry, Faculty of Medicine, Erciyes University, Kayseri, Turkey.

OBJECTIVES:Laboratory-related errors are important part of the medical errors. Clinicians usually consider laboratory test results as absolute values. However, laboratory tests have pre-analytical, analytical and biological variations (BV). The aim of this study was to search how the knowledge and developments produced in the laboratory medicine were used by clinicians for the benefit of the patients. For this purpose, we selected BV as a model and investigated how clinicians use BV data to interpret test results.

MATERIALS and METHODS:A survey comprising 399 clinicians was conducted to evaluate knowledge regarding the BV in Turkey. We prepared a questionnaire consisting 9 questions. Five questions were open ended and 2 of the open ended questions were case based. A scoring system A to D (A indicates correct interpretation and $\mathrm{D}$ indicates clinician has no knowledge on variations) were used to evaluate open ended questions.

RESULTS:Clinicians (46\%) used combination of the reference interval, clinical evaluation and literature data to interpret test results. In open-ended questions $83 \%$ of clinicians scored C or D. None of the clinicians were using the reference change value in monitoring test results. Clinicians did not read an article about BV $(88.3 \%)$ and they were not trained about BV $(82 \%)$.

CONCLUSIONS:Clinicians are not adequately familiar with the new developments in laboratory medicine and do not use BV data to interpret tests results. Effective communication/collaboration between laboratorians and clinicians will enable clinicians to interpret laboratory tests accurately and use BV data more efficiently.

Keywords: Biological Variation, Interpretation of test results, Laboratory-Clinic Interaction
0-062

Using the model of quality indicators: A pilot study

Oğuzhan Zengi ${ }^{1}$, Derya Sönmez ${ }^{2}$, Bağnu Orhan ${ }^{2}$, Cihan Coşkun ${ }^{3}$, Hümeyra Emre ${ }^{4}$, Doğan Yücel ${ }^{5}$

${ }^{1}$ Medical Biochemistry Laboratory, Bağcılar Research and Training Hospital, Istanbul, Turkey

${ }^{2}$ Medical Biochemistry Laboratory, Istanbul Research and Training Hospital, Istanbul, Turkey

${ }^{3}$ Medical Biochemistry Laboratory, Haydarpaşa Research and Training Hospital, Istanbul, Turkey

${ }^{4}$ Medical Biochemistry Laboratory, Necip Fazıl City Hospital, Kahramanmaraş, Turkey

${ }^{5}$ Medical Biochemistry Laboratory, Ankara Research and Training Hospital, Ankara, Turkey

OBJECTIVES:Continuous monitoring of laboratory performances is a key activity in identifying errors and promoting improvement in Laboratory Medicine. Since 2008, The Working Group "Laboratory errors and Patient safety (WG-LEPS) of the International Federation of Clinical Chemistry and Laboratory Medicine (IFCC) has designed a Model of Quality Indicators (MQI) and implemented an informative platform to collect QI from laboratories worldwide. Data collected are processed, and a report describing the laboratory results compared with those of other participating laboratories is periodically issued. A working group is established in Turkish Biochemical Society to use of these quality indicators and to contribute harmonisation studies. The aim of the study was to determine usage of MQI on practical area.

MATERIALS and METHODS:Bağcılar Training and Research Hospital Laboratory has been chosen to enter their own data for MQI project. After registered to the project web site, data entering has been started since may 2017. There were nine selected QIs, four QI were about pre-analytical phase and five QIs post-analytical phase.

RESULTS:WG-LEPS publishes performance reports for each indicator as annualy. According to these published reports our laboratory data which are entered to the system meet high and medium performance criteria.

CONCLUSIONS:There are lot of QIs that are offered. One of the biggest challenges is the difficulty in understanding some indicators. Quality indicators should be translated to all languages and there should be more detailed explanation and calculation methods. Due to difficulties in obtaining data from laboratory information system, a common midware is needed.

Keywords: quality indicators, wg-leps, laboratory errors, patient safety

\section{O-063}

National guidelines for the preparation, distribution and testing of purified water for clinical laboratories

Oytun Portakal, Suat Kucuk, Oguzhan Zengi, Enver Sarıgul, Mehmet Gonen,

Canan Topçuoğlu, Arzu Kosem, Sabri Evren, Dilek Guven, Settar Kosova,

Dogan Yücel

Hacettepe University Medical School, Department of Biochemistry, Turkey

OBJECTIVES:The aim was to prepare a national guide for the preparation, distribution, and testing of purified laboratory water in clinical laboratories in Turkey.

MATERIALS and METHODS:Laboratory Water Guide Working Committee was established by Turkish Biochemical Society in 2017. It contained seven clinical biochemists. After appointing director, the treasurer, technical experts, method scientists and editors were defined. The needs for the country were determined. The strategic plan was made. The partners were identified. After that, work management was established; literature and resources were reviewed. The study was started. Total twelve meetings were performed in different cities. The study completed in one and a half year. After evaluation and approval, it was reported and the guide book was published in June 2019.

RESULTS:This national guideline defines the Laboratory Water Purification System (LWPS). It covers the subjects of laboratory water types and quality characteristics, water pollutants, laboratory water purification methods, storage and distribution, validation and monitoring of the water system and sampling and testing of laboratory water. Laboratory water types and quality characteristics were described based on CLSI. The guide defines minimum laboratory water quality standards to be considered and the processes to improve water quality in clinical laboratories. It also includes the problems that may be encountered 
during design, and the solutions. LWPS should be operated under control and maintained. Thus, it ensures operational stability and meets water quality control standards.

CONCLUSIONS:This guide is national, and provides to clinical biochemists the information about operation, storage, monitoring and use of laboratory water system.

Keywords: Laboratory Water Purification System, Clinical Laboratories, Turkish Biochemical Society, Working Committee

\section{O-064 \\ Evaluation of CKD-EPI Pakistan equation for estimated glomerular filtration rate (EGFR) in Pakistan}

Sibtain Ahmed, Lena Jafri, Aysha Habib Khan

Section of Chemical Pathology, Department of Pathology and Laboratory

Medicine, The Aga Khan University, Karachi Pakistan

OBJECTIVES:To evaluate the results of 24-hour urinary creatinine clearance $(\mathrm{CrCl})$ with estimated glomerular filtration rate (eGFR) using the Chronic Kidney Disease Epidemiology Collaboration (CKD-EPI), CKD-EPI Pakistan (CKD-EPI Pak), Cockcroft Gault (CG) and 4-variable Modification of Diet in Renal Disease (MDRD) equations.

MATERIALS and METHODS:A descriptive, cross-sectional study was conducted at the section of Clinical Chemistry, Department of Pathology and Laboratory Medicine, The Aga Khan University, Karachi. Laboratory data of subjects' $\geq 18$ - $<70$ years ordering 24 -hour urinary $\mathrm{CrCl}$ was retrieved. Statistical comparison of eGFR using CKD-EPI, CKD-EPI Pak, CG and MDRD with the timed urine collection $\mathrm{CrCl}$ was done using regression analysis.

RESULTS:The mean age of the group $(n=670)$ was $51.3 \pm 15.4$ years with a median of 53 (IQR:22.3) years, $55.7 \%$ being males. Median BMI of males and females was $26.98 \mathrm{~kg} / \mathrm{m} 2$ (IQR: 7.09 ) and $26.16 \mathrm{~kg} / \mathrm{m} 2$ (IQR: 6.97), respectively. Mean GFR using 24-hour creatinine clearance was $57.1 \pm 35.9 \mathrm{ml} / \mathrm{min} / 1.73 \mathrm{~m} 2$ with a median of $51 \mathrm{ml} / \mathrm{min} / 1.73 \mathrm{~m} 2$. Urinary creatinine clearance showed strong correlation with CG, MDRD, CKD-EPI and CKD-EPI Pak, showing $\mathrm{r}=0.78$, $r=0.79, r=0.82$, and $r=0.82$, respectively. Sensitivity was highest for the CKDEPI Pakistan (84.7\%). Similarly, CKD-EPI Pakistan equation showed the highest agreement $(88.7 \%)$ with $\mathrm{CrCl}$ compared to the other formulae.

CONCLUSIONS:The CKD-EPI Pak equation is more accurate and precise than the CG, CKD-EPI and MDRD in estimating GFR in Pakistani population. The results of this study support automated reporting of eGFR using CKD-EPI Pak equation in laboratories across Pakistan.

Keywords: estimated glomerular filtration rate, equations, Pakistan, adults

\section{O-065 \\ The local technical validation of Barricor ${ }^{\mathrm{TM}}$ tube that uses a mechanical separator}

Kamil Taha Uçar ${ }^{1}$, Neval Aksoy ${ }^{1}$, Belgin Erhan $^{2}$, Berrin Berçik Inal ${ }^{1}$

${ }^{1}$ Department of Medical Biochemistry, Istanbul Gaziosmanpasa Taksim Training and Research Hospital, Istanbul, Turkey

${ }^{2}$ Department of Physical Medicine and Rehabilitation, Istanbul Gaziosmanpasa Taksim Training and Research Hospital, Istanbul, Turkey

OBJECTIVES:Unsuitable samples are common problems for laboratories. The blood collection tubes need to be validated and verified prior to routine laboratory administration in order to reduce this problem. In this study, we aimed at comparing the technical qualifications of routinely used BD Serum Separator II/SST IITM tubes with BD Barricor ${ }^{\mathrm{TM}} \mathrm{LH}$ tubes for local technical validation. MATERIALS and METHODS:150 volunteers were enrolled in the study. Samples were collected in two tubes by a single phlebotomist. 12 quality indicators were evaluated. The difference $(\%)$ was calculated with the formula proposed by EFLM. In case of any difference of less than $1 \%$ for indicators, the evaluated tube was considered adequate.

RESULTS:Indicators, such as tubes with physical defects of manufacturing, with no vacuum or that fail to create vacuum, not properly fitting into the blood collection device, under filling $(10 \%)$, cracked tubes or tubes with leaking from the cap before/after centrifugation, blood contamination of collection device, haemolysed specimens, incorrect positioning of separator after centrifugation, tubes including fibrin strand/mass in sample after centrifugation, red blood cell adhesion to interior tube walls after centrifugation were found adequate in Barricor ${ }^{\mathrm{TM}}$ tubes. White particulate matter (WPM) was observed in $24.6 \%$ of Barricor ${ }^{\mathrm{TM}}$. Therefore, the last indicator, tubes including gel/foreign material/ WPM in sample after centrifugation, was found inadequate in Barricor ${ }^{\mathrm{TM}}$.

CONCLUSIONS:It was thought that WPM with $24.6 \%$ presence would not cause any interference in a properly filled tube. Thus, Barricor ${ }^{\mathrm{TM}}$ was found to be technically adequate. Technical validation studies should be encouraged in terms of total quality management.

Keywords: Phlebotomy; blood collection devices; preanalytical errors; technical validation; quality management

\section{O-066}

The utility of preanalytical quality indicators: A Turkish survey study

Bağnu Orhan ${ }^{1}$, Derya Sönmez ${ }^{1}$, Hikmet Can Cubukçu ${ }^{2}$, Oğuzhan Zengi ${ }^{3}$, Hümeyra Emre ${ }^{4}$, Ipek Çınaroğlu ${ }^{5}$, Murat $\mathrm{Keleş}^{6}$, Alper Gümüş ${ }^{7}$, Cihan Coşkun ${ }^{8}$ ${ }^{1}$ İstanbul Training and Research Hospital

${ }^{2}$ Erzurum Mareşal Çakmak Devlet Hastanesi

${ }^{3}$ İstanbul Bağcılar Training and Research Hospital

${ }^{4}$ Kahramanmaraş Necip Fazıl City Hospital

${ }^{5}$ Becton Dickinson Life Sciences-Preanalytical Systems

${ }^{6}$ Bursa Public Health Laboratory

${ }^{7}$ İstanbul Başakşehir State Hospital

${ }^{8}$ İstanbul Haydarpaşa Numune Training and Research Hospital

OBJECTIVES:The utility of quality indicators(QIs) to monitor the total testing process of laboratories is able to improve the quality of services and enhance patient safety. The present study set out to investigate the harmony between the preanalytical QIs within the context of Model of Quality Indicators (MQI) determined by IFCC Working Group on Laboratory Errors and Patient Safety (WG-LEPS) and the QIs used by Turkish medical biochemistry laboratories. The other purpose of this investigation is to assess the usability of IFCC preanalytical QIs considering the conditions of Turkey.

MATERIALS and METHODS:A survey consisting of 9 questions prepared by Turkish Biochemical Society Working Group of Laboratory Errors and Patient Safety was applied to 81 laboratories via Survey Monkey.

RESULTS:According to survey results, 91 percent of participant laboratories used QIs proposed by Ministry of Health Quality Standards in Health. While some QIs within the context of MQI were utilized by over 80 percent of laboratories, some of other QIs were used by under 10 percent of laboratories. CONCLUSIONS:The majority of the laboratories utilized QIs determined by Ministry of Health Quality Standards in Health and Ministry of Health On-site Assessment Guide. These standards were found to be partially compatible with IFCC WG-LEPS QIs. The inability of the health information system (HIS) limits the usage of QIs proposed by WG-LEPS. Education of medicial biochemistry specialists and other healthcare personnel and improvement of HIS are crucial for the QIs usage. Definitons of QIs should be more plain and understandable.

Keywords: laboratory errors, preanalytic phase, patient safety, quality indicators

\section{O-067}

A web-based application for management of quality control data

Deniz Ilhan Topcu $^{1}$, Merve Sibel Güngören ${ }^{2}$

${ }^{1}$ Department of Biochemistry, Faculty of Medicine, Başkent University, Ankara, Turkey

${ }^{2}$ Düzen Laboratories Group, Ankara, Turkey

OBJECTIVES:In medical laboratories, internal quality control (IQC) is the main quality control measure to assess the analytical phase. The main role of IQC is to detect errors in the analytical mom to assure patent safety. Regulations also obligate laboratories to conduct an IQC scheme. The main gap in IQC is the evaluation of results and existing laboratory information systems (LISs) mainly lack state of the art evaluation methods for QC data. Although there are some advanced software solutions for management of QC data, license costs can be a burden for laboratories. The aim of this study is to develop an open source web application for evaluation of quality control results.

MATERIALS and METHODS:In this study, a web-based QC data management 
software was developed by using R programming language. Shiny tools were used for user-friendly interface. For sigma metrics calculation, different recommendations for quality requirements were utilized. For this software, QC data can be extracted from either LIS or middleware.

RESULTS:General features of this software are evaluation with given target values, revision of target values from accumulated data, evaluation with multi rules, calculation of bias from EQA results, calculation of total error and sigma metrics. This software can be applicable for daily IQC monitoring, periodic reporting and analytics.

CONCLUSIONS:This web-based software provides a more accessible way to correct QC practices for clinical laboratories.

Keywords: Quality control, internal quality control, data management software, open source

\section{O-068 \\ Quality control application for CBC parameters by 'Average of Normals' method}

Ilknur Alkan Kuşabbi, Neslihan Cihan, Emel Çolak Samsum, Mehmet Şeneş, Vildan Fidanc1, Doğan Yücel

Department of Medical Biochemistry, Ankara Health Training and Research Center, Health Sciences University, Ankara

OBJECTIVES:Application of internal quality control (IQC) with 'average of normals $(\mathrm{AON})$ method for complete blood count $(\mathrm{CBC})$ parameters and to validate $\mathrm{AON}$ method with biased data.

MATERIALS and METHODS:For this study, CBC results in a Sysmex XNseries instrument in our hospital's hematology laboratory in July 2019 were evaluated. AON method was applied using Sysmex XbarM program. XbarM is based on calculation of weighted moving averages derived from Bull's algorithm. Using XbarM 'target/limit' function, laboratory-specific target values were calculated automatically. When target and \% values specified for control limits were entered into XbarM, weighted averages were calculated for each batch of results. A simulation study was performed with the biases of $-80 \%,-50 \%,-20 \%$,$10 \%,-5 \%,-2 \%, 2 \%, 5 \%, 10 \%, 20 \%, 50 \%, 100 \%$ on the existing patient data. Using Excel, based on formula used by XbarM, new moving averages were calculated and evaluated for each bias point. Averages were taken every 50 patients. Target values for our laboratory were calculated automatically by monitoring patient results, control limits were determined according to \% values recommended by Sysmex.

RESULTS:During follow-up, instrument had 2-8 AON points daily. There was no value exceeding control limits and this was consistent with conventional IQC. When biases were added, AON method could not detect $2 \%$ bias in general, but $5 \%$ and above could be detected in early period.

CONCLUSIONS:AON method is cost-effective and complementary to conventional IQC programs especially to detect systematic errors.

Keywords: Average of Normals, Moving Averages, Quality Control, CBC

\section{O-069 \\ Evaluation of the most common rejection reasons in the preanalytical process at our laboratory using six sigma analysis}

Mehmet Akif Bozdayı, Seren Orhan, Mustafa Örkmez, Mehmet Tarakçıŏlu Deparment of Biochemistry,Gaziantep University, Turkey

OBJECTIVES:Based on statistical calculations, "Six Sigma Level" provides information about process performance In our study, we aimed to determine the most common reason for rejection of the samples rejected in the preanalytical period and to evaluate the effect of corrective and preventive actions to reduce this rate by using six sigma level analysis. MATERIALS and METHODS:The quality studies which were conducted in 2019 at our laboratory showed that the most common reason for rejection in preanalytical period was inadequate sample collection in sedimentation test. Educational programme was organized for the related units to correct this ratio. After the educational training, sedimentation test rejection rates were reexamined and evaluated.

RESULTS:When the distribution of rejection reasons was examined, it was found that the highest rate was inadequate sample collection the rate was $28.92 \%$.the most frequently rejected test was ESR test with a rate of $55 \%$. Educational program was provided to related units. One month sigma values were calculated before and after educational training. in June 2019(pre-education) sedimentation test total number was: 7455, rejected sample number: 418, sigma level was 3.1. in July 2019 (after the educational program has been applied ) total number of samples was 9534 , rejected sample number: 335 sigma level was determined as 3.4 .

CONCLUSIONS:The analysis of the main cause of preanalytical problems in the laboratory and the initiation of corrective and preventive actions increase the quality of the laboratory. We believe that sigma values will improve significantly by increasing the frequency of the educational training programs.

Keywords: Preanalytic phase, rejection rates

\section{O-071 \\ Analytical performance of Cobas 6500 for predicting urinary tract infection}

Esra Firat Oğuz ${ }^{1}$, Arzu Sakall11 ${ }^{1}$, Ipek Mumcuoğlu², Ortaç Ateș ${ }^{1}$

${ }^{1}$ Clinical Biochemistry Laboratory, Ankara City Hospital, Ankara, Turkey

${ }^{2}$ Clinical Microbiology Laboratory, Ankara City Hospital, Ankara, Turkey

OBJECTIVES:Urinary tract infection (UTI) is the most common disease in the community. Urinalysis is the most requested screening test in patients with symptoms possible UTI. We aimed to compare the dipstick and sediment analysis results of fully automated Cobas 6500 urine analyser with gold standard urine culture results.

MATERIALS and METHODS:Data of 571 patients with order for urine dipstick test, urine sediment analysis and urine culture between March and November 2018 were evaluated retrospectively. Sensitivity, specificity, positive and negative predictive values and ROC curve analysis was performed for leukocyte esterase (LE) and white blood cells (WBC).

RESULTS:349 of 571 patients had positive urine culture results. The sensitivity of dipstick leukocyte esterase was found to be $73.35 \%$, whereas the specificity was $61.71 \%$. Positive and negative predictive values were $75.07 \%$ and 59.56 , respectively. WBCs showed $70.77 \%$ sensitivity with $65.31 \%$ specificity with positive and negative predictive values of $76.23 \%$ and 58.7 , respectively. The area under the curve (AUC) for LE and WBC were 0.707(0.668-0.744) and 0.753(0.716-0.788).

CONCLUSIONS:Leukocyte esterase in urine dipstick test and microscopic WBC tests had comparable results in predicting UTI. Clinical decisions based on dipstick urine and sediment analysis could be both time and cost effective and may reduce the need for the conventional urine culture.

Keywords: Cobas 6500, leukocyte esterase, urine culture, WBC

\section{O-072}

A comparison of Sysmex UF-5000 flow cytometer and Fuchs-Rosenthal Chamber in urine sediment analysis

Ozlem Unay Demirel

Bahcesehir University, Faculty of Medicine, Medical Biochemistry, Turkey

OBJECTIVES:Urine analysis is a basic test in the clinical laboratory. Urine sediment analysis is a part of urine analysis that gives laboratory professionals valuable information. Since manual examination is the gold standard for analysis it is time consuming and work-intensive procedure. In this study we aimed to compare the performance of Sysmex UF-5000 flow cytometer with the manual Fuchs-Rosenthal chamber in terms of urine sediment analysis.

MATERIALS and METHODS:A total of 127 fresh urine samples from outpatient clinics are analyzed. We used Sysmex UF-5000 flourescence flow cytometer for urine analysis and Fuchs-Rosenthal chamber for urine sediment analysis.We compared two methods by using Passing-Bablok regression analysis, Pearson correlation coefficient ( $\mathrm{r}$ ) and Bland-Altman bias plot.Statistical analysis was performed using Analyse-it software version 3.80 (Analyse-it Software,Ltd., Leeds, UK), Microsoft Excel 2010, and CLSI Statis-Pro software version 3.0. RESULTS:A good correlation was observed between manual and automated white blood cell (WBC) counts in all urine samples. $(\mathrm{r}=0.988 ; \mathrm{y}=1,162 \mathrm{x}+$ $0,489 ; \mathrm{n}=127)$. UF-5000 demonstrated a significant proportional overestimation with Passing-Bablok regression (95\% CI slope: 1,110 to 1,226). For red blood 
cell (RBC) counts, correlation between UF-5000 and the counting chamber was observed in all samples $(\mathrm{r}=0,966 ; \mathrm{y}=1,1 \mathrm{x}+0,75)$.

CONCLUSIONS:This study showed us that urine analysis with flow cytometers is a very promising area and with automation getting more commonly used in clinical laboratories in the world,it is likely to replace the manual microscopy and thus reduce the workload and also time and energy needed in laboratories. Keywords: Urine analysis, flow cytometer, Fuchs-Rosenthal chamber

\section{O-073}

Determination of serum carbamazepine by tandem mass spectrometry

Duygu Eryavuz Onmaz ${ }^{1}$, Sedat Abusoglu ${ }^{1}$, Ali Unlu ${ }^{1}$, Abdullah Sivrikaya $^{1}$, Gülsüm Abusoglu²

${ }^{1}$ Selcuk University Faculty of Medicine, Department of Biochemistry, Konya, Turkey

${ }^{2}$ Department of Medical Laboratory Techniques, Selcuk University Vocational School of Health, Konya, Turkey

OBJECTIVES:Carbamazepine is a first-line drug for the treatment of different forms of epilepsy. Therapeutic range of carbamazepine in plasma is 5 to $10 \mu \mathrm{g} / \mathrm{ml}$; more specifically, $7.4 \mu \mathrm{g} / \mathrm{ml}$ for adults and $8.2 \mu \mathrm{g} / \mathrm{ml}$ for children. Carbamazepine plasma level is directly correlated with dose, therapeutic effect and side effects. Carbamazepine plasma level is affected by several factors. It is altered by age and pregnancy status including several other factors. Individualization of drug dose with the help of plasma level detection is a must in case of carbamazepine therapy. In this study, our aim was to develop a LC-MS/ MS method for the measurement of carbamazepine.

MATERIALS and METHODS:100 $\mu \mathrm{L}$ of the internal standard (gliclazide solution) on a standard solution or sample of $250 \mu \mathrm{L}$ was vortexed for 30 s by adding $500 \mu \mathrm{L}$ of acetonitrile included $\% 0.1$ formic acid, followed by centrifugation at $12000 \mathrm{rpm}$ for $10 \mathrm{~min}$. The supernatants were taken into glass tubes and evaporated with nitrogen gas. The residue was dissolved in $200 \mu \mathrm{L}$ of in the mixture of acetonitrile:water $(50: 50 ; \% \mathrm{v}: \mathrm{v})$ then enjected into LC-MS/MS system.

RESULTS:The calibration curve for carbamazepine was established at a range of 0.15 to $80 \mu \mathrm{g} / \mathrm{ml}$. Detection limit and quantitation limit for carbamazepine; 0.15 $\mu \mathrm{g} / \mathrm{ml}$ and $0.3 \mu \mathrm{g} / \mathrm{ml}$, respectively. The retention time was determined 1.62.Total run time was 5 minutes.

CONCLUSIONS:We can conclude that the developed method can be useful for clinical studies and routine therapeutic drug monitoring with the desired precision and accuracy.

Keywords: Tandem mass spectrometry, drug monitoring, carbamazepine

\section{O-074}

\section{D placental barrier models: A novel cryogel based method}

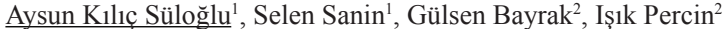

${ }^{1}$ Department of Biology, Section of Zoology, Hacettepe University, Ankara, Turkey

${ }^{2}$ Department of Biology, Section of Molecular Biology, Hacettepe University, Ankara, Turkey

OBJECTIVES:Cryogels are formed below the freezing point of the solvent. Their advantages are their inherent interconnected three dimentional (3D) macroporous structure and utilization as a scaffold in tissue engineering. At the same time, cryogels supply biocompatible property, ECM support and will represent in vivo better. Poly (2-hydroxyethyl methacrylate-glycidyl metacrylate) [p(HEMAGMA)] cryogels are super-macroporous (10-100 $\mu \mathrm{m})$, hydrophilic cryogels. They provide non-specific protein interactions at the minimum level, are mechanically and chemically stable and they are resistant to microbial and enzymatic reactions. In tissue engineering studies, fibronectin potentially increases the cell adherence and folic acid improves the viability of cells.

MATERIALS and METHODS:In this study, galactose containing p(HEMAGMA)/gelatin cryogels were synthesized and then fibronectin or folic acid was attached on the surface. The proliferative and adhesive effects were investigated by seeding BeWo human placental choriocarcinoma cell line on the cryogels. Cryogels was characterized by swelling test and scanning electron microscopy (SEM). The viability of the BeWo cells on different crogels were investigated by
Alamar blue assay $48 \mathrm{~h}$ after incubation.

RESULTS:BeWo cells and cryogels have been observed to interact well and cells were proliferated successfully. Among the cryogel groups, p(HEMA-GMA)/ gelatin-folic acid group had the highest cell viability. Cell viability was lower in galactose-bound cryogel groups than in galactose-free groups.

CONCLUSIONS:This novel placenta model reflects the in vivo more precisely and can be used as a model in transport of xenobiotics and their metabolites such as newly developed and mandatory drugs used in pregnancy and also for cosmetics, cleaning products, food additives, nanoparticules.

Keywords: placenta, cryogel, scaffold, cell viability

\section{O-075 \\ Antioxidant effects of flavonoid neoeriocitrin on streptozotocin-induced INS-1E cell diabetic model}

Elif Karacaoğlu, Eymen Ece Aldemir, Güldeniz Selmanoğlu

Department of Biology, Faculty of Science, Hacettepe University, Ankara, Turkey

OBJECTIVES:Diabetes mellitus is a common metabolic disease, and its prevalance has been increasing globally. Numerous studies have revealed that generation of reactive oxygen species play a crucial role in diabetes. Moreover increasing in DNA damage also is a cause of experimental diabetes. Flavonoids have been promising therapy especially for preventing diabetes mellitus. Neoeriocitrin is a flavonoid which is found in citrus species and it could be a promising agent in preventing $\beta$-cells against diabetes. Our aim in this study is to reveal the antioxidant effects of neoeriocitrin against STZ-induced diabetes model in INS-1E cells.

MATERIALS and METHODS:INS-1E cells (rat insulinoma cell line) were preincubated with neoeriocitrin at various concentrations $(0,0.25,0.5,1 \mu \mathrm{M})$ for 21 hours then $5 \mathrm{mM}$ streptozotocin (STZ) were added into cells and incubated for 3 hours. STZ, which is a DNA alkylating agent, was used for inducing diabetes in INS-1E cells. Antioxidant ezymes such as superoxide dismutase (SOD) and catalase (CAT) were measured in cell lysates by spectrophometrically. Additionally, oxidized guanine species as a marker of DNA/RNA oxidative damage was measured spectrophotometrically.

RESULTS:STZ-induced INS1-E cells showed elevated SOD activity and decreases in CAT activity. Antioxidant status of the cells changed by neoeriocitrin treatment. DNA/RNA oxidative damage increased by STZ treatment, and neoeriocitrin caused changes in oxidized guanine species.

CONCLUSIONS:Biologically flavonoids have potential to reduce free radicals and risk of diabetes. Neoeriocitrin which has an antioxidant activity is a promising agent, however further studies are needed to exert mechanism of action against reactive oxigen species in INS-1E cells. This research was financially supported by Hacettepe University Scientific Projects Coordination Unit (Project No: FBA2018-16746).

Keywords: Neoeriocitrin, flavonoid, diabetes, INS-1E cells, oxidative stress

\section{O-076}

In vitro investigation of Argiope bruennichi derived spider silk materials

$\underline{\text { Seçil Karahisar Turan }}{ }^{1}$, Aysun Kılıç Süloğlu ${ }^{1}$, Tuncay Türkeş², Semra Ide Nurhayat Barlas ${ }^{1}$

${ }^{1}$ Department of Biology, Hacettepe University, Ankara, Turkey

${ }^{2}$ Department of Biology, Niğde Ömer Halisdemir University, Niğde, Turkey

${ }^{3}$ Department of Physics Engineering, Hacettepe University, Ankara, Turkey

OBJECTIVES:Spider silks' exceptional chemical and mechanical properties lead to extensive researches in both industry and medicine. The purpose of this study is to investigate the effects of a novel designed biological material, the dragline silk of Argiope bruennichi, in surgical applications. As a first step towards this purpose, in vitro cytotoxicity assays were performed.

MATERIALS and METHODS:A. bruennichi specimens were collected from the Eastern parts of Black Sea region. Special made dragline silking system was used to collect the spider silk material for filament preparation. Nanoscopic structure analyses of the silk filaments were done by SAXS (Small Angle X-ray Scattering) and WAXS (Wide Angle X-ray Scattering) methods. Abinitio 3D nanoscale morphologies were also obtained by using SAXS data. 
Cytotoxic potentials of spider silk based suture materials and their nanocomposite/biopolymer coated (film/filament) forms were investigated in L929 fibroblast cells by MTT (3-(4,5-Dimethyl-2-thiazolyl)-2,5-diphenyl-2Htetrazolium bromide) and LDH (Lactate dehydrogenase) assays.

RESULTS:SAXS analyses indicate that biopolymer coating causes the most stable 3D nanoglobular aggregations while the nanocomposite coating is convenient to keep the natural nanostructures of the filaments. The results of in vitro studies showed that dragline silk of $A$. bruennichi and nanopowdered levan coated films of $A$. bruennichi silk filaments did not show any cytotoxic effect on L929 cells.

CONCLUSIONS:According to the results of in vitro studies, Argiope bruennichi silk has a potential usage area in surgical applications. Further in vivo studies will be proceeded to investigate the effects of these silk materials on wound healing process in a dorsal skin flap model on rats.

Keywords: spider silk, levan, nanopowder, MTT, LDH

\section{O-077}

\section{MicroRNAs in patients with type 2 diabetic nephropathy}

Kadriye Akpinar ${ }^{1}$, Diler Aslan ${ }^{1}$, Semin Melahat Fenkçi², Vildan Caner $^{3}$ ${ }^{1}$ Department of Medical Biochemistry, Pamukkale University, Denizli, Turkey ${ }^{2}$ Department of Endocrinology and Metabolic Diseases, Pamukkale University, Denizli, Turkey

${ }^{3}$ Department of Medical Genetics, Pamukkale University, Denizli, Turkey

OBJECTIVES:To evaluate the associations of the diabetic nephropathy (DN) with 10 miRNAs that were found to be related to diabetes in human (miR-21a3p, miR-29a-3p, miR-29b-3p, miR-29c-3p, miR-126-3p, miR-192-5p and miR320c) and animal (miR-129-1-3p, miR-137, miR-212-3p) studies.

MATERIALS and METHODS:Plasma miRNAs were analyzed by RT-PCR and correlations with the eGFRs were evaluated in 50 healthy controls (male: $\mathrm{n}=24$; age:55 \pm 11 ; female: $\mathrm{n}=26$; age:54 \pm 13 ) and 100 type 2 diabetics (T2DM) (male: $\mathrm{n}=46$; age: $60 \pm 11$; female: $\mathrm{n}=54$; age:56 \pm 11 ). Diabetics were divided into normoalbuminuric (NAlb, $\mathrm{n}=51$ ), microalbuminuric (MicAlb, $\mathrm{n}=25)$ and macroalbuminuric (MacAlb, $n=24$ ) groups. Forty-nine diabetics were diagnosed as DN.

RESULTS:miR-21 were detected in approximately half of all groups, and found significant $(p<0.05)$ decreases in the T2DM, DN and MacAlb than those in the controls (T2DM: 5-fold, DN: 7, MacAlb: 7). The decrease in miR-192 that were detected in all groups was found significant $(\mathrm{p}<0.05)$ (T2DM: 2-fold, DN:2.4). The eGFR-based on cystatin $\mathrm{C}$ showed positive correlations $(\mathrm{p}<0.05)$ with miR-21, miR-192 and miR-126 (r=0,262, $\mathrm{r}=0,203$, and $\mathrm{r}=0,417$, respectively). miR-21 and miR-29c were correlated $(\mathrm{p}<0.05)$ with MDRD eGFR $(\mathrm{r}=0,243$ and $\mathrm{r}=0,188$, respectively). The correlation of CKD-EPI-creatinine with miRNA-192 was significant $(r=0,185, p=0,023)$. miR-21, miR-192, miR-29c and miR-320 were negatively correlated $(\mathrm{p}<0.05)$ with microalbuminuria $(\mathrm{r}=-0,323, \mathrm{r}=-$ $0,267, \mathrm{r}=-0,173$ and $\mathrm{r}=-0,172$, respectively). miR-21 and miR-192 were found to be significant in distinguishing the $\mathrm{DN}$ from healthy subjects (AUC $=0,726$, $\mathrm{p}=0,0001$ and $\mathrm{AUC}=0,717, \mathrm{p}=0,0001$, respectively).

CONCLUSIONS:miR-21 and miR-192 could be related to DN. More research is needed for the association of miR-29 family, miR-126, miR-212 and miR-320c with DN.

Keywords: Diabetic nephropathy, microRNA, miR-21, miR-192

\section{O-078}

Differentiation of osteopetrotic IPSC to osteoclasts: Comparison of osteopetrotic\& healthy osteoclast

$\underline{\text { Inci Cevher }}^{1}$, Berna Alkan ${ }^{1}$, Duygu Uçkan Çetinkaya ${ }^{2}$, Fatma Visal Okur ${ }^{2}$ ${ }^{1}$ Stem Cell Department, Institute of Health Sciences, Hacettepe University, Ankara, Turkey. Center for Stem Cell Research and Development-PediSTEM, Hacettepe University, Ankara, Turkey.

${ }^{2}$ Stem Cell Department, Institute of Health Sciences, Hacettepe University, Ankara, Turkey. Center for Stem Cell Research and Development-PediSTEM, Hacettepe University, Ankara, Turkey.Faculty of Medicine Pediatric Hematology / Bone Marrow Transplantation Unit, Hacettepe University, Ankara, Turkey.
OBJECTIVES:Malign infantile osteopetrosis is a lethal, rare genetic bonedisease characterized by dysfunctional-osteoclasts. The only available treatment for this disease is hematopoietic stem cell transplantation.

MATERIALS and METHODS:The main-purpose of this study was to evaluate the osteoclast-dysfunction using osteopetrotic-IPSC-derived-osteoclasts. Firstly,iPSC-lines from both TCIRG1-mutation positive osteopetrosis-patients and a healthy-donor were differentiated into hematopoietic stem cells-(iHSCs), which were characterized with colony-forming-capacity and CD34+CD45+surfacemarkers by flow-cytometry. Immunophenotyping was evaluated for myeloidimmnunuphenotyping. Then iHSCs were differentiated into myeloid-progenitors followed by osteoclast differentiation using M-CSF,RANK-L. Immunophenot yping,immunofluorescence(IF)-staining,Scanning-electron-microscope(SEM) analysis, and gene-expression profile related to functionality and maturation of iPSCs-derived-osteoclasts were performed.

RESULTS:All iPSC-lines were differentiated succesfully into iHSCs. PatientiHSCs were showed to have three-times more CFU-M potential comparing to donor, and BFU-E potential was observed only donor-iHSCs. OsteopetroticiPSCs-derived-osteoclasts were stained weakly-positive with Cathepsin-K,and Rhodamine-phalloidin comparing to the control. Osteopetrotic-iPSC-derivedosteoclasts were positive for TRAP, imagined as giant-multinucleated-cells, and over $95 \%$ of the cells were CD14+CD16+, and CD18+CD51/61+..SEMimages showed that there was a difference between the size of podosomes of patient-and donor-osteoclasts. Osteopetrotic-osteoclasts showed a delayed expression of related functional-genes compared to controls. At the end of differentiation osteopetrotic-osteoclasts showed significantly reduced expression of Cathepsin-K, Calcitonin-R, and NFATC1 genes comparing to controls. All results indicate that both donor-and osteopetrotic-IPSCs were differentiated into osteoclasts, but osteopetrotic-osteoclasts showed different gene and protein expression patterns, and size of podosomes indicating a disease-specific functional impairment. Functionality analyses are ongoing.

CONCLUSIONS:The results of our study might help to increase our knowledge about normal-and osteopetrotic-osteoclastogenesis, but needs to be supported with more detailed functionality-analyses.

Keywords: Osteopetrosis, Osteoclasts, TCIRG1, Induced Pluripotent Stem Cells

\section{O-079 \\ Monodisperse-porous metal oxide microspheres with peroxidase/oxidase mimetic activity as a new tool for biomolecule determination}

Burcu Gökçal ${ }^{1}$, Sevim Eda Öğ̈̈t ${ }^{2}$, Rukiye Babacan Tosun ${ }^{3}$, Çiğdem Kip ${ }^{1}$, Özlem Hamaloğlu ${ }^{1}$, Ali Tuncel ${ }^{1}$

${ }^{1}$ Department of Chemical Engineering, Hacettepe University, Ankara, Turkey ${ }^{2}$ Division of Bioengineering, Hacettepe University, Ankara, Turkey

${ }^{3}$ Division of Nanotechnology and Nanomedicine, Hacettepe University, Ankara, Turkey

OBJECTIVES:Peroxidase-oxidase mimetic activity materials are important for developing new commercial assays.

MATERIALS and METHODS:A new staged-shape templated hydrolysis and condensation protocol was developed for the synthesis of monodisperse-porous metal oxide microspheres.The magnetic forms were synthesized with the accessible magnetic hematite $\left(\mathrm{Fe}_{3} \mathrm{O}_{4}\right)$ nanoparticles tightly immobilized within the porous interiors of microspheres.Magnetic $\mathrm{SiO}_{2}$ microspheres and plain $\mathrm{CeO}_{2}$ microspheres exhibited peroxidase mimetic activity while oxidase-mimetic activity was obtained with the plain $\mathrm{MnO}_{2}$ microspheres.

RESULTS:3,3',5,5'-tetramethylbenzidine (TMB) and o-phenylenediamine (OPDA) were used as the synthetic substrates providing products via oxidase/ peroxidase-like activities of corresponding microspheres, whose formation kinetics were followed by UV-Vis spectroscopy or fluorescence spectroscopy. The interaction of plain and ligand-attached forms of porous metal oxide microspheres with the biomolecules resulted in a change in the peroxidaseoxidase mimetic activity which was proportional to the biomolecule concentration in the interaction medium, due to the selective adsorption of biomolecule onto the surface of microspheres.

CONCLUSIONS:The concentration of ascorbic acid was determined via oxidaselike activity of plain, porous $\mathrm{MnO}_{2}$ microspheres, using both colorimetric and fluorometric protocols. The ligand attached forms of plain $\mathrm{SiO}_{2}$ microspheres were evaluated for developing a colorimetric assay for the determination of histidine tagged protein concentration. We believe that the methodology 
exemplified here will be effectively used for developing new commercial assays for the estimation of concentrations of various agents in biological samples. The research was supported by Hacettepe University Scientific Research Projects Coordination Unit contract numbered as FBA-2019-17337, entitled " Development of a peroxidase-microzyme-based biosensor for protein determination". The financial support provided is gratefully acknowledged. Keywords: Peroxidase activity, Kit fabrication, DNA, Protein, Ascorbic acid.

\section{O-080 \\ Evaluation of analytical process performance by Six Sigma methods in laboratories}

Dilek Iren Emekli $^{1}$, Nergiz Zorbozan ${ }^{2}$, Zübeyde Erbayraktar $^{3}$

${ }^{1}$ Erbayraktar Private Medical Laboratories,Izmir, Turkey

${ }^{2}$ Department of Biochemistry, Kemalpaşa State Hospital, İzmir, Türkiye

${ }^{3}$ Department of Biochemistry, Dokuz Eylul University Medical Faculty, Izmir,

Turkey

OBJECTIVES:Clinical laboratories are responsible for producing reliable, reproducible and accurate test results.Six sigma is a quality managemen strategy that enables evaluation of processes, identification and improvement of defects. In this context, the aim of our study is to evaluate the analytical process performance of routine tests in our laboratory with six sigma method.

MATERIALS and METHODS:Internal quality control(IQC) data of tests were obtained retrospectively. Mean, standard deviation and coefficient of variation values of IQC were calculated. Process sigma values were calculated using the formula "\%Allowable Total Error (TEa)- Bias\%/CV\%". TEA values were determined according to CLIA 88 . Sigma $\leq 3$ was considered as low quality, sigma between 3 and 6 was considered as good quality and Sigma $\geq 6$ was considered as world class quality.

RESULTS:In the tests we evaluated, all of the process sigma were $>3$. The sigma levels of IQC1 for albumin, creatinine, LDL, urea, chloride,total cholesterol,HDL and sodium and IQC2 sigma for albumin, urea, UIBC, chloride, creatinine, potassium, sodium, direct bilirubin tests were between 3-6. The sigma of IQC1 for ALP, ALT, AST, CK, CKMB, 1ron, u1bc, phosphorus, GGT, glucose, calcium, $\mathrm{LDH}, \mathrm{Mg}$,potassium, total protein, triglyceride, uric acid, amylase, lipase, direct bilirubin, total bilirubin, CRP, ferritin and sigma of IQC2 for ALP, ALT, AST,CK,CK-MB, rron, phosphorus,GGT, glucose, calcium, total cholesterol, HDL,LDL, LDH, magnesium, total protein, triglyceride, uric acid, amylase, lipase, total bilirubin, CRP, ferritin tests were $\geq 6$.

CONCLUSIONS:Six Sigma Methodology is a very effective method for assessing the laboratory's analytical process performance.In our study, the performance of our laboratory was found to be good or worldclass.

Keywords: six sigma, internal qality control, Allowable Total Erro

\section{O-081}

Evaluation of analytical quality of cardiac biomarkers in the emergency laboratory by sigma metrics

\section{Saadet Kader}

Karapinar State Hospital Biochemistry Laboratory Karapınar, Konya, Turkey

OBJECTIVES:The Six-Sigma Methodology is a quality measurement method in order to evaluate the performance of the laboratory. In the present study, we aimed to evaluate the analytical performance of our emergency laboratory by using the internal quality control data of cardiac biomarkers and by calculating process sigma values

MATERIALS and METHODS:Biological variation database (BVD) are used for Total Allowable Error (TEa). Sigma values were determined from coefficient of variation $(\mathrm{CV})$ and bias resulting from Internal Quality Control (IQC) results for 4 subsequent months. If the sigma values are $\geq 6$, between 3 and 6 , and $<3$, they are classified as »world-class «, »good « or »un -acceptable«, respectively.

RESULTS:Whenthesigmavalueswereanalyzedbycalculatingthemeanof4months, Troponin I (cTnI), CKMB mass, Myoglobin $(\mathrm{Mb})$ were found $<3$.

CONCLUSIONS:The "poor quality" levels of cTnT, CKMB mass, Myoglobin sigma values, decision is taken for the improvement of cardiac markers in our laboratory. It is possible to determine the test with high error probability by evaluating the fine sigma levels and the tests that must be quarded by a stringent quality control regime. In clinical chemistry laboratories, an appropriate quality control scheduling should be done for each test by using Six-Sigma Methodology. Keywords: Six Sigma, total allowable error, bias, Cardiac Biomarkers

\section{O-083}

Automated Vitamin D immunoassay comparison with LC-MS/MS method

Ercan Saruhan $^{1}$, Muhittin Serdar ${ }^{2}$

${ }^{1}$ Department of Medical Biochemistry, Muğla Sıtkı Koçman University, Muğla, Turkey

${ }^{2}$ Department of Medical Biochemistry, Acıbadem University, Istanbul, Turkey

OBJECTIVES:Serum 25-hydroxy (25-OH) vitamin D is the major form of vitamin $\mathrm{D}$ and the best indicator of vitamin $\mathrm{D}$ status in human beings. In this study, we compared analytical performance of automated immunoassay method, Roche Elecsys Vitamin D total assay, with the liquid chromatography tandem mass spectrometry (LC-MS/MS).

MATERIALS and METHODS:A total of 80 samples were used to assess vitamin D analytical perfomance. Vitamin D levels were determined in Roche Cobas E602. Results were classified into three groups; vitamin D concentration of less than $20 \mathrm{ng} / \mathrm{mL}$ (LOW, $\mathrm{n}=20$ ), 20-50 ng/mL (NORMAL, $\mathrm{n}=41$ ), and >50 ng/ $\mathrm{mL}(\mathrm{HIGH}, \mathrm{n}=19)$. Serums were stored at $-80{ }^{\circ} \mathrm{C}$ for 2 weeks until LC-MS/MS analysis. Regression analysis and Bland-Altman plots were used for comparison between methods.

RESULTS:The correlation for all samples was acceptable $(n=80, r=0,961)$. The $\mathrm{r}$ value was higher in samples with low vitamin $D$ levels $(n=20, r=0.948)$ as compared to those with normal vitamin $\mathrm{D}$ values $(\mathrm{n}=41, \mathrm{r}=0.902)$ and high vitamin $\mathrm{D}$ values $(\mathrm{n}=19, \mathrm{r}=0.715)$. The mean percent difference of Elecsys was $-2.6 \%$ compared to LC-MS/MS. The results were linear with slope of 1.055 , intercept of 0.833 $\mathrm{ng} / \mathrm{mL}$, a correlation coefficient of 0.961 , and a mean bias of $-2.6 \%(\mathrm{P}<0,0001)$ CONCLUSIONS:Our data show that the Roche Elecsys Vitamin D Total Assay has good correlation with LC-MS/MS. Although the LC-MS/MS method is considered reference method, it needs a special instrument and personnel and is thus expensive. Therefore, Roche's automated immunoassays for vitamin D total assay is more suitable for evaluating vitamin D status.

Keywords: 25 -hydroxyvitamin D, correlation, analytical perfomance, electrochemiluminescence, liquid chromatography tandem mass spectrometry

\section{O-084 \\ Calculation of measurement uncertainty of three different biochemistry parameters}

Seren Orhan, Mehmet Akif Bozdayı, Mustafa Örkmez, Mehmet Tarakçığlu Department of Biochemistry,Gaziantep University,Gaziantep,Turkey

OBJECTIVES:Measurement uncertainty is a quality indicator which is used to show the distribution level of the test result. In principle, two approaches can be used to calculate measurement uncertainty: "bottomtop" and "top-down".The "top-down" approach uses laboratory test performance information, such as intra-laboratory and inter-laboratory quality control data to estimate uncertainty associated with the test results. The aim of this study; to calculate the measurement of uncertainty values of three biochemistry parameters separately using internal and external quality control data. then these values will be compared with the CLIA's\% total permissible error $(\%$ TEa) values.

MATERIALS and METHODS:In this study, the uncertainty estimation of serum ALP GGT CK levels based on the "top-down" approach described in the Nordest guideline was used as a practical example. The tests were performed by enzymatic method on Beckman Coulter AU5800 analyzer.

RESULTS:SerumALPGGTandCKanalysis measurementuncertainty was found to be ALP: $18.74 \%$, GGT: $13.3 \%$, CK: $17.7 \%$ in the $95 \%$ confidence interval,and these values did not exceed the CLIA\% TEa (ALP:\% 20,GGT:\%15,CK:\%20) values. The $\% \mathrm{CV}$ values of the tests were ALP control level 1\% CV: 7,07 level $2 \% \mathrm{CV}$ : 5,36, GGT level $1 \% \mathrm{CV}: 2,36$ level $2 \% \mathrm{CV}: 3,45$, CK level $1 \% \mathrm{CV}: 5.58$ level $2 \% \mathrm{CV}: 2.9$ and bias values were determined as bias(ALP): 0.6 , bias(GGT): 2.8 , bias(CK): 4.79 .

CONCLUSIONS:The uncertainty value is a parameter that increases confidence in the accuracy of the measurement results. Therefore, laboratories should 
provide results that do not exceed the target measurement uncertainty values. Keywords: Measurement uncertainty, Accuracy

\section{O-085 \\ Development of a LC/MSMS method for quantification of adrenal-derived 11-oxygenated 19-carbon steroids}

\author{
Ali Yaman \\ Department of Clinical Biochemistry, Marmara University Pendik Education \& \\ Research Hospital, Istanbul, Turkey
}

OBJECTIVES:Recent studies have shown that adrenal-derived 11-oxygenated 19-carbon (110xC19) steroids may be associated with congenital adrenal hyperplasia $(\mathrm{CAH})$ as well as premature adrenarche, polycystic ovary syndrome and castration resistant prostate cancer. In our study, we measured $11 \beta$-hydroxyandrostenedione (11OHA4) and 11 $\beta$-hydroxytestosterone (11OHT) metabolites, which are most likely of adrenal origin, by a LC/MSMS method. In addition, we thoroughly validated our method and evaluated whole steroid profile in patients who have 21-hydroxylase deficiency (21OHD) which accounts for the majority of CAH cases.

MATERIALS and METHODS:11OHA4 and 11OHT standards (Steraloids, USA) and 11OHA4-d7 internal standard (Cambridge Isotopes, USA) were used in the preparation of the calibrators and internal standard working solution, respectively. Plasma samples were prepared by liquid-liquid extraction (LLE). Poroshell 120 EC-C18 column $(50 \times 2.1 \mathrm{~mm}, 2.7 \mu \mathrm{m}$; Agilent Technologies, USA) was used and the analysis time was set as $15 \mathrm{~min}$. Precursor and product ions for 11OHA4 (303.2>121.0, 267.0), 11OHT (305.3>121.0, 269.0) and 11OHA4-d7 $(310.43>128.0,243.0)$ were determined.

RESULTS:The linear measuring range of method was determined as $0.1-20.0$ $\mathrm{ng} / \mathrm{mL}$ for $11 \mathrm{OHA} 4$ and $50-1000 \mathrm{pg} / \mathrm{mL}$ for $11 \mathrm{OHT}$. The $\% \mathrm{CV}$ values of the upper and lower limits of the measuring interval were $<15 \%$. Two 11 oxC19 steroids were significantly higher in $210 \mathrm{HD}$ patients $(n=7)$ compared to controls $(\mathrm{n}=56)(3-5$-fold, $\mathrm{P}<0.0001)$.

CONCLUSIONS:Our findings suggest that 11 oxC19 steroids might serve as an additional biomarker in patients with 21OHD. LC/MSMS methods which have unique advantages like permitting more flexibility in application of new biomarkers are considered as reference methods for measuring steroid hormones. Keywords: 11-Oxygenated 19-Carbon Steroids, congenital adrenal hyperplasia

\section{O-086}

Structural bioinformatics approach in bioactive peptide research: Tomato vicilin case study

Burcu Kaplan Türköz, Bahar Bakar, Canan Kartal, Semih Ötleş Department of Food Engineering, Faculty of Engineering, Ege University, İzmir, Türkiye

OBJECTIVES:Bioactive peptides (BAP) are gaining importance due to their proven health benefits. Food proteins are valuable sources for BAPs with antihypertensive (ACE inhibitory), antidiabetic (DPP-IV inhibitory), antioxidant and antimicrobial activities. There are several bioinformatics tools used in identification of BAPs based on amino acid sequence of the protein and the digestion patterns of different proteases. However, these tools do not take into account the tertiary structure of the protein of interest and therefore can not accurately predict the peptides which will be released under experimental conditions.

MATERIALS and METHODS:Tomato seed proteins were extracted using a modified protocol and were analyzed using electrophoresis. The structure of Solanum lycopersicum (tomato) vicilin was modeled based on the experimental structure of Solanum melongena vicilin using I-TASSER and RaptorX. The models were visualized and analyzed using PyMol Graphics.

RESULTS:Here, we present a structural biology approach to predict BAP release from tomato seed proteins. Among the extracted proteins, vicilin was selected for further analysis. The structure of vicilin was modeled and was subjected to in silico structure based proteolysis. Our approach takes into account the surface accessibility of specific cleavage sites of enzymes; carboxyl terminus of lysine or arginine for trypsin and large hydrophobic or aromatic side chains for chymotrypsin. The resulting peptides are further evaluated using BIOPEP for their ACE and DPP-IV inhibitory activities.
CONCLUSIONS:BAPs are very useful considering their therapeutic potential. Structure based approaches will shed light on time consuming experimental studies in order to produce targeted peptides. Acknowledgements: This work is supported by TÜBİTAK(117O319) Keywords: Bioactive peptides, Protein Structure, Food proteins

\section{O-087 \\ In silico prediction of antidepressant-binding sites on human glutathione reductase}

Kerem Teralı, Özlem Dalmızrak, Hamdi Öğüş, Nazmi Özer Department of Medical Biochemistry, Faculty of Medicine, Near East University, 99138 Nicosia, Cyprus

OBJECTIVES:Antidepressants, which are available worldwide, represent widely used treatments for major depressive disorder. They are grouped into various classes of compounds with slightly different mechanisms of action. Glutathione reductase (GR; EC 1.6.4.2) is a homodimeric enzyme that plays a crucial role in the regeneration of the antioxidant "recharger" glutathione (GSH) from glutathione disulfide (GSSG). While reduced antioxidant capacity is associated with many pathologies, cancer cells are known to use diverse strategies to increase their antioxidant capacity. For this reason, GR inhibition is expected to have divergent consequences in human health and disease.

MATERIALS and METHODS:Here, using protein-ligand docking and interaction profiling as well as ligand (un)binding simulations, we aim at predicting the mode of interaction between human GR and six antidepressants: two selective serotonin reuptake inhibitors (fluoxetine and sertraline); two tricyclic antidepressants (amitriptyline and clomipramine); and two alternative or nontraditional antidepressants (hypericin and pseudohypericin). We evaluate our in silico data according to in vitro results from enzyme kinetic studies previously conducted and reported by our research group.

RESULTS:All the antidepressants in question appear to be accommodated well in an eccentric cavity located in between the two monomers of GR. Hypericin and pseudohypericin, with their large rigid hydrophobic ring systems, bind the enzyme with the highest predicted affinity.

CONCLUSIONS:Overall, these interactions may subject healthy cells and tissues to oxidative stress. On the positive side, however, they may guide medicinal chemists in the search for new anticancer drugs.

Keywords: antidepressants, human glutathione reductase, computational biology, oxidative stress, anticancer drugs

\section{O-088}

\section{Smart approval service for biochemical tests}

Begum Mutlu ${ }^{1}$, Abdullah Elci², Ali Ozen Akyurek ${ }^{3}$, Ersen Uzunal ${ }^{3}$ ${ }^{1}$ Gazi University, Department of Computer Engineering, 06570, Ankara, TURKEY

${ }^{2}$ Istanbul Provincial Health Directorate, Maltepe (Number 3) Public Health Laboratory, Istanbul, TURKEY

${ }^{3}$ Ventura Software Inc., Ankara, TURKEY

OBJECTIVES:Biochemical tests' results have been approved manually by clinical laboratory experts for decades. This process causes a remarkable loss of time for experts; it is highly likely that some of test results are mistakenly approved/unapproved, since massive amounts of test results are produced in every passing moment. This study aimed to automatically evaluate the test results by handling the problem as binary classification in artificial intelligence (AI). MATERIALS and METHODS:Utilized AI models were data-driven machine learning (ML) approaches (Naïve Bayesian (NB), Support Vector Machine (SVM), Multi-layer Perceptron (MLP)) and expert-cooperated Fuzzy Systems (FSs). Dataset containing total 28 type of biochemical tests employed in one month-period was obtained from Istanbul Provincial Health Directorate, Maltepe (Number 3) Public Health Laboratory. There were 379340 manually approved and 3568 unapproved tests. Considered properties of tests were test-result, gender-of-patient, age-of-sample, has-interference, distance-to reference-range, delta-check, average-of-median, test-repeated, has-previous-test, and previoustest-result.

RESULTS:5-fold cross-validation was applied for each test type and ML 
method. Performance evaluation was based on area under the Receiver Operating Characteristic curve (ROC-AUC). Among the ML approaches, NB dramatically outperforms SVM and MLP. Although FSs have lower data-dependency, FS's classification capability was on par with NB (ROC-AUC: 0.9997-0.9996). CONCLUSIONS:Approval of test results by an automatic decision-making mechanism is essential need to make rapid, efficient and standardized evaluation. In this study, the feasibility of automatic approval process was empirically investigated. The best classification capability was obtained by NB and FSs. This research was supported by TUBITAK regarding project "3180740-PROBO Smart Approval System".

Keywords: Biochemical tests, laboratory approval system, artificial intelligence

\section{O-089}

\section{Evaluation of saliva Kallikrein-8 levels related with stress}

Rabia Semsi ${ }^{1}$, Umut Kökbaș ${ }^{2}$, Aylin Sepici Dinçel ${ }^{1}$, Erdal Ergünol ${ }^{3}$,

Levent Kayrın ${ }^{2}$

${ }^{1}$ Department of Biochemistry, Faculty of Medicine, Gazi University, Ankara,

Turkey

${ }^{2}$ Department of Biochemistry, Faculty of Medicine, Kyrenia University,

Kyrenia, TRNC

${ }^{3}$ Faculty of Dentistry, Cyprus Health and Social Sciences University, Morphou, TRNC

OBJECTIVES:Kallikreins are a group of serine proteases that are enzymes capable of cleaving peptide bonds in proteins. It is a proteolytic enzyme that mediates the conversion of kininogen ( $\alpha 2$-globulin) to brandykinin. Kallikrein is found in blood, lymph, saliva and various external secretions. The aim of this study was to determine whether KLK8 changes in saliva due to stress.

MATERIALS and METHODS:The study included 22 students (15 female / 7 male) from Faculty of Dentstry of Cyprus University of Health and Social Sciences, Term-I. General and dental health of the students were evaluated in the appropriate anamnesis format. Saliva samples were collected between 08.00-09.00 am in the morning, before the exam (12.00) and after the exam (14.00-15.00). It was collected by SARSTEDT brand saliva collection tubes as recommended. Kallikrein levels were measured by KLK 8 Human ELISA kit (pg/mL).

RESULTS:Body mass index and mean age (years) of the students were 20.4 \pm 0.93 and $21.6 \pm 3.46$, respectively. In the present study, salivary kallikrein- 8 levels were determined morning, before and after the exam. There were a statistically significant differences between salivary samples of kallikrein- 8 values in the morning and before/after exam ( $p<0.001, \mathrm{p}<0.001$, respectively). However, there was no statistically significant difference between salivary kallikrein- 8 before and after the exam $(\mathrm{p}=0.985)$. Electrobiochemical studies are ongoing to confirm the results

CONCLUSIONS:These results show that KLK8 changes in saliva due to stress. Keywords: Kallikrein-8, Saliva, Stress

\section{O-090 \\ The evaluation of ADAMTS-1 and ADAMTS-13 levels at coronary collateral circulation}

Abdülhakim Hasan Gül ${ }^{1}$, Dilek Ülker Çakır ${ }^{1}$, Sema Uysal $^{1}$,

Müşerref Hilal Şehitoğlu' ${ }^{1}$, Bahadır Kırılmaz², Emine Gazi², Mehmet Arslan²

${ }^{1}$ Department of Medical Biochemistry, Çanakkale Onsekiz Mart University,

Çanakkale

${ }^{2}$ Department of Cardiology, Çanakkale Onsekiz Mart University, Çanakkale

OBJECTIVES:Coronary collateral circulation (CCC) plays important roles at coronary artery disease. It was revealed that ADAMTS-1, ADAMTS-13 and VEGF are included at angiogenesis and arteriogenesis. We aimed to show the relationship between ADAMTS-1, ADAMTS-13, VEGF levels and grade of CCC.

MATERIALS and METHODS:The patients who were applied to underwent coronary angiography according to European Society of Cardiology guide, were included in study. Collateral degree was graded according to Rentrope Cohen classification. Patients who had grade 0-1 collateral vessels were classified poorly-developed collateral group; grade 2-3 collateral vessels were classified well-developed collateral group. VEGF, ADAMTS-1 and ADAMTS-13 levels were measured by ELISA.

RESULTS:From patients who had $>\% 90$ obstruction at Coronary Angiographic View,36 patients were at well-developed collateral group and 33 patients were at poorly-developed collateral group. There has been no stastically significant difference between groups ADAMTS-1, ADAMTS-13 and VEGF levels $(p=0,428, p=0,577, p=0,450)$. On the other hand, ADAMTS- 1 levels were lower in well-developed collateral group $(6.6 \pm 6.4)$ than poorly-developed collateral group (9.6 \pm 11.9$)$.

CONCLUSIONS:According to our results, VEGF levels of patients with Coronary Artery Disease (CAD) were higher than the normal population. In addition, plasma VEGF level seems not to be associated with development of CCC. The alteration of ADAMTS-1 might have role the formation of CCC, but ADAMTS-13 level may not be associated with development of CCC. Our data highlighted that ADAMTS-1 and ADAMTS-13 molecules cannot be used as predictor marker for CCC. Further studies with more participants will elucidate the role of ADAMTS-1 and ADAMTS-13 on the development of CCC.

Keywords: ADAMTS-1. ADAMTS-13. Coronary Collateral Circulation

\section{O-091}

Apelin and other adipokines as potential biomarkers in myocardial ischemia

Mehmet Ali Gul ${ }^{1}$, Ebubekir Bakan ${ }^{1}$, Zafer Bayraktutan $^{1}$,

Nurcan Kilic Baygutalp ${ }^{2}$, Muhammet Celik ${ }^{1}$, Murat Ozmen³,

Neslihan Yuce ${ }^{1}$, Nuri Bakan ${ }^{1}$

${ }^{1}$ Faculity of Medicine, Department of Medical Biochemistry, Ataturk University, Erzurum, Turkey

${ }^{2}$ Faculity of Pharmacy, Department of Biochemistry, Ataturk University,

Erzurum, Turkey

${ }^{3}$ Faculity of Medicine, Department of Cardiology, Ataturk University, Erzurum, Turkey

OBJECTIVES:Apelin is an endogenous peptide and ligand of the G proteincoupled receptor (also known as the APJ receptor). Apelin plays role in cardiovascular systems and participates in pathological processes for heart failure, obesity, diabetes. Apelin is called new adipokine and can be secreted by fat cells. Adipose tissue express and secrete numerous adipokines. CTRP1 and CTRP9 novel members of the adipokine family, have intersecting functions in the regulation of lipid metabolism and contribute to cardiovascular protection. In this study, we investigated the association of serum levels of apelin, CTRP1 and CTRP9 with patients with and without myocardial ischemia after myocardial perfusion scintigraphy.

MATERIALS and METHODS:This study was carried out in 44 patients with myocardial ischemia and 44 patients without myocardial ischemia after myocardial perfusion scintigraphy. Serum apelin, CTRP1 and CTRP9 levels measured with ELISA method, whole blood HbA1c measured with HPLC method.

RESULTS:According to t test results for groups; there was a statistically significant difference between the groups in serum apelin, CTRP1, CTRP 9 levels and whole blood HbA1c levels. Serum apelin, CTRP1, CTRP9 levels and whole blood $\mathrm{HbA1}$ c levels were found statistically significant between groups ( $\mathrm{p}$ $=0.050, \mathrm{p}=0.045, \mathrm{p}=0.043$ and $\mathrm{p}=0.001$, respectively).

CONCLUSIONS:Our data showed serum apelin, CTRP1 and CTRP9 could be used as potential biomarkers or supportive parameters for myocardial ischemia, and $\mathrm{HbAlc}$, consequently diabetes, may be predisposing factor for myocardial ischemia.

Keywords: apelin, ctrp1, ctrp9, hba1c 
XXVII. Balkan Clinical Laboratory Federation Meeting BCLF 2019

XXX. National Congress of the Turkish Biochemical Society TBS 2019

\section{O-092}

Relationship between platelet activating factor acetylhydrolase and cardiac valvular calcification in dialysis patients

Serkan Bolat ${ }^{1}$, Vildan Fidanci ${ }^{1}$, Deniz Elcik ${ }^{2}$, Ozdem Kavraz Tomar ${ }^{3}$,

Sani Namik Murat ${ }^{2}$, Murat Duranay ${ }^{3}$, Dogan Yucel ${ }^{1}$

${ }^{1}$ Department of Medical Biochemistry, University of Health Sciences, Ankara

Training and Research Hospital, Ankara, Turkey

${ }^{2}$ Department of Cardiology, University of Health Sciences, Ankara Training and Research Hospital, Ankara, Turkey

${ }^{3}$ Department of Nephrology, University of Health Sciences, Ankara Training and Research Hospital, Ankara, Turkey

OBJECTIVES:The cardiovascular mortality risk has increased seriously in Chronic Kidney Disease (CKD), especially dialysis patients. PAFacetylhydrolase is an enzyme that hydrolyzes platelet activating factor (PAF). Atherosclerosis was associated with valvular calcifications and PAF-AH. So we investigated the association of PAF-AH activities with valvular calcification in dialysis patients.

MATERIALS and METHODS:92 patients treated with hemodialysis (HD) and peritoneal dialysis (PD) and $86 \mathrm{CKD}$ patients which were divided into five groups according to GFR [grade 1-2(>60), grade $3 \mathrm{a}(45-59)$, grade $3 \mathrm{~b}(30-44)$, grade $4(15-29)$ and grade $5(<15)]$ were included in the study. Echocardiography was performed to assess valvular calcification. We analysed urea, creatinine, uric acid, protein, albumin, ALT, ALP, calcium, phosphate, magnesium, cholesterol, triglyceride, HDL, 25-OH D vitamine, iPTH, CRP, NT-proBNP in serum and protein, albumin, creatinine in urine. PAF-AH activity was determined by color change based on the reaction of DTNB with free thiols which formed due to of 2-thioPAF by PAF-AH and measured at $412 \mathrm{~nm}$ by a rate method.

RESULTS:There was no significant difference between the PAF-AH activities of dialysis and control groups $(p>0.05)$. Higher PAF-AH activities in HD patients were associated with both valvular calcification and aortic valvular calcification $(p<0.05)$. There was no association between PAF-AH and calcification in PD and control groups.

CONCLUSIONS:In addition to anti-inflammatory and antioxidative properties of PAF-AH, proatherogenic and proinflammatory products formed by enzyme complicates the interpretation of activity changes. Findings in our study suggest that the elevation of PAF-AH activities in HD patients are associated with valvular calcification particularly in aortic valve involvement.

Keywords: Hemodialysis, peritoneal dialysis, PAF-AH, calcification

\section{O-093}

Determination of ADMA and ghrelin levels as a marker of endothelial dysfunction in asthma patients

Cumhur Bilgi ${ }^{1}$, Emre Avci² ${ }^{2}$ Burcu Baba ${ }^{1}$, Gülçin Alp Avci²

${ }^{1}$ Department of Medical Biochemistry, Faculty of Medicine, Yüksek İhtisas University, Ankara, Turkey

${ }^{2}$ Department of Molecular Biology and Genetics, Faculty of Arts and Sciences, Hitit University, Çorum, Turkey

OBJECTIVES:Asthma is a chronic inflammatory disorder defined as obstruction and hyperresponsiveness of airways. Appetite modulating hormone ghrelin plays a role in various diseases associated to inflammation. Ghrelin regulates secretion of proinflammatory cytokines and induces anti-inflammatory profile, although the underlying mechanisms remain elusive. Nitric oxide (NO) plays important roles such as airway smooth muscle relaxation, airway mucus secretion, and host defense in the lung. Asymmetric dimethylarginine (ADMA) that is defined as a marker of endothelial dysfunction is a competitive inhibitor of NO synthesis. To date, the role of ADMA in the pathogenesis of asthma has not been elucidated completely. The aim of the study was to evaluate ADMA and ghrelin levels in asthmatic patients compared to healthy subjects.

MATERIALS and METHODS:Thirty-eight asthma patients and twenty five healthy controls were included in the study. The patient group was constituted according to the Global Initiative for asthma guidelines. Serum ADMA levels were determined by HPLC and ghrelin levels were measured by ELISA.

RESULTS:Serum ADMA levels were significantly higher in the patients compared to controls $(\mathrm{p}=0.014, \mathrm{p}<0.05)$. The median ADMA levels were found about $0.56 \mu \mathrm{mol} / \mathrm{L}$ in patient group. In contrast, ghrelin levels $(116.24 \pm 2.03 \mathrm{pg} /$ $\mathrm{ml})$ were lower in the patients compared to the controls $(154.3 \pm 21.6 \mathrm{pg} / \mathrm{ml}$, $\mathrm{p}<0.05)$.

CONCLUSIONS:Our findings demonstrated that increased ADMA and decreased ghrelin levels may be contributed to asthma pathophysiology. Our data support the idea that asthmatic patients have risk of endothelial dysfunction for cardiovascular diseases. However, more studies are required to elucidate the molecular mechanisms of ADMA as well as ghrelin actions in asthma. Keywords: Asthma, asymmetric dimethylarginine, endothelial dysfunction, ghrelin

\section{0-094}

Antimicrobial and antioxidant activities of Lactarius deliciosus

Emre Avcı, Elif Sevinç, Gulcin Alp Avci

Hitit University, Faculty of Arts and Sciences, Department of Molecular Biology and Genetics, Corum,Turkey

OBJECTIVES:Fungi, which are of particular importance in the ecosystem because of their biodegradable properties, are known to be an important source of biologically active components of both food and medical value. Fungal extracts are used in the treatment and prevention of many diseases. Lactarius deliciosus mushroom is also known as Kanlica mushroom and is an edible mushroom with high nutritional value. The aim of this study was to determine the antioxidant and antimicrobial activities of water, ethanol and cloform extracts of Lactarius deliciosus.

MATERIALS and METHODS:Antioxidant activities of the extracts were determined by DPPH (2,2-diphenyl-1-picrylhydrazase) method. Antimicrobial activities against Escherichia coli, Candida albicans, Staphylococcus aureus, Enterococcus faecalis, Pseudomonas aeruginosa were determined by disc diffusion method.

RESULTS:The water and ethanol extracts of Lactarius deliciosus were found to have antioxidant activity. And also antimicrobial activity was determined according to the solvent used and the type of microorganism. The most susceptible strain was P. aeruginosa and the most resistant strain was E.coli. CONCLUSIONS:Mushrooms are highly important due to their properties such as fat, vitamins, carbohydrates and proteins. It is important to evaluate these fungi in terms of antioxidant and antimicrobial activity.

Keywords: Lactarius deliciosus, Antimicrobial Activity, Antioxidant Activity

\section{O-095}

Telmisartan and irbesartan alleviate methylglyoxal-induced elevation of MG-H1 in VSMCs

\section{Mustafa Kırça}

Department of Medical Biochemistry, Kütahya Health Sciences University, Kütahya, Turkey

OBJECTIVES:Methylglyoxal (MGO) is a glycolysis by-product and was found elevated in diabetics. It was described as the most powerful glycating agent which results in enhanced AGEs formation. MGO-derived the most important physiological AGE is hydroimidazolone 1 (MG-H1). Telmisartan and irbesartan were suggested to be protective against MGO. The aim of this study was to evaluate the effects of telmisartan and irbesartan on MGO-induced MGH1 levels under low and high glucose media in vascular smooth muscle cells (VSMCs), those play a prominent role in vascular diseases.

MATERIALS and METHODS:Primary cultured VSMCs were isolated from rat aorta. MGO-treated cells $(200 \mu \mathrm{M})$ were incubated in low and high glucose media for 48 hours with or without telmisartan or irbesartan (both $10 \mu \mathrm{M}$ ). MGH1 was measured by ELISA technique as triplicates.

RESULTS:MGO raised MG-H1 concentration in low and high glucose media. High glucose alone elevated MG-H1 levels similar to MGO treatment in low glucose media. While telmisartan and irbesartan did not mitigate MGO-induced MG-H1 increase in low glucose media, they both displayed a significant reduction in MG-H1 concentration in high glucose media. Though telmisartan seemed better to reduce MG-H1 level, there was not any significant difference between telmisartan and irbesartan.

CONCLUSIONS:Our findings showed that telmisartan and irbesartan were effective against MGO-induced MG-H1 concentration increase in high glucose media. The reason could arise from MG-H1 level was highest in MGO-induced 
cells cultured in high glucose. Further studies supporting our data are needed and these may guide clinicians to choose the best antihypertensive medicine in diabetic patients.

Keywords: methylglyoxal, MG-H1, telmisartan, irbesartan, high glucose.

\section{O-096 \\ Reelin enzyme levels in suicide or self harm attempt emergency \\ service patients}

Turgut Dolanbay ${ }^{1}$, Mustafa Y1lmaz ${ }^{2}$, Mehtap Gurger ${ }^{2}$, Metin Atescelik ${ }^{2}$,

Mehmet Cagri Goktekin ${ }^{2}$, Nevin Ilhan ${ }^{3}$, Huseyin Fatih Gul ${ }^{4}$

${ }^{1}$ Department of Emergency Medicine, Kafkas University Health Research and Application Hospital, Kars, Turkey

${ }^{2}$ Department of Emergency Medicine, Firat University School of Medicine,

Elazig, Turkey

${ }^{3}$ Department of Medical Biochemistry, Faculty of Medicine, Firat University,

Elazig, Turkey

${ }^{4}$ Department of Medical Biochemistry, Faculty of Medicine, Kafkas University, Kars, Turkey

OBJECTIVES:Suicide is an important public health problem in our country as in the whole world. Although studies have shown that Reelin enzyme plays a role in the pathophysiology of neuropsychiatric diseases, information on how these enzyme levels change in suicidal behaviour remains unclear. For this reason, we aimed to investigate the enzyme levels of Reelin in self-harm or suicidal patients. MATERIALS and METHODS:This work includes 86 consecutive suicide patients who applied to Frat University, Faculty of Medicine, Emergency Medicine Department and 100 healthy age and sex-matched control. Reelin levels were analysed in serum samples in accordance with the ELISA kit procedure. In addition, the effect of demographic data such as body mass index (BMI) and age on Reelin levels were also investigated.

RESULTS:While in the suicide patient group Reelin enzyme level was 3038.31 $\mathrm{ng} / \mathrm{L}$, it was $2271.20 \mathrm{ng} / \mathrm{L}$ in the healthy control group. When the demographic data were compared with Reelin enzyme levels, it was found that Reelin enzyme levels were negatively correlated with both BMI $(r=-0.298, p<0.001)$ and age $(\mathrm{r}=-0.362, \mathrm{p}<0.001)$.

CONCLUSIONS:It was suggested that increases in serum Reelin enzyme levels may be helpful in the diagnosis of suspicious suicidal cases. Interestingly, people with lower BMI and younger age have higher Reelin levels which calls for further research. When elaborating results on suicidal patients, it should be remembered that too many environmental and/or social factors are effective in suicidal behavior.

Keywords: Suicide, Reelin, Emergency Service, ELISA

\section{O-097}

Relationship between lipoprotein(a) and other lipids in children

$\underline{\text { Fatime Merdan }}^{1}$, Seydi Ali Peker ${ }^{2}$, Serkan Bolat $^{3}$, Dogan Yucel $^{4}$ ${ }^{1}$ Department of Medical Biochemistry, Dr. Sami Ulus Obstetrics, Child Health and Diseases Training and Research Hospital, Ankara, Turkey

${ }^{2}$ Department of Medical Biochemistry, Yüksek Ihtisas Hospital, Kırıkkale,

Turkey

${ }^{3}$ Department of Medical Biochemistry, Dogubayazit Dr. Yashar Eryilmaz State Hospital, Agri, Turkey

${ }^{4}$ Department of Medical Biochemistry, Ankara Training and Research Hospital, Ankara, Turkey

OBJECTIVES:Lipoprotein(a) (Lp(a)) levels are hereditary and essentially depend on the Apo(a) gene located on chromosome 6. Lp(a) may cause inflammation, oxidative stress, fibrinolysis and plaque instability. The aim of this study was to determine the correlation of $\mathrm{Lp}$ (a) with total cholesterol (TC), HDL-cholesterol (HDL-C), LDL-cholesterol (LDL-C) and trIglycerides (TG) in children.

MATERIALS and METHODS:The study included 25 patients with Lp(a) $>100$ $\mathrm{mg} / \mathrm{dL}, 25$ patients with $50-100 \mathrm{mg} / \mathrm{dL}, 25$ patients with $30-50 \mathrm{mg} / \mathrm{dL}$ and 25 healthy children for the control group. Fasting blood $\mathrm{Lp}(\mathrm{a})$ was measured by immunoturbidimetric method and TC, TG, HDL-C was measured by photometric method in Beckman Coulter AU5800. LDL-C was calculated by the Friedewald formula.

RESULTS:In this study, Lp(a), TC, LDL-C, TG were significantly higher in all patient groups compared to the control group $(p<0.001)$. There was no significant difference in HDL-C $(\mathrm{p}>0.05)$. Only Lp(a) showed statistical difference between the groups $(\mathrm{p}<0.001)$. No significant difference was found in lipid profile $(\mathrm{p}>0.05)$. $\mathrm{Lp}(\mathrm{a})$ showed a weak positive correlation with TC, LDL-C and TG $(r=0.340, p<0.001 ; r=0.326, p<0.001 ; r=0.275, p<0.001)$. CONCLUSIONS:Lp(a) is an independent risk factor for premature cardiovascular disease. But also it shows a correlation with other cardiovascular risk factors such as TC, LDL-C and TG.

Keywords: Lipoprotein(a), LDL-cholesterol, triglycerides

\section{O-098}

Relationship between B-HCG and LUC\% levels

Funda Eren

Ankara City Hospital, Ankara, Turkey

OBJECTIVES:In this study, we aimed to investigate the changes in hematological parameters in pregnancy.

MATERIALS and METHODS:Hematology parameters of pregnant (521 patients) $(17180 \pm 3652 \mathrm{mIU} / \mathrm{mL})$ and non-pregnant (498 patients) $(6.23 \pm 0.91$ $\mathrm{mIU} / \mathrm{mL}$ ) women were compared. BHCG levels were determined with Siemens Centaur XP and hematological parameters were analysed with ADVIA 2120i Hematology System.

RESULTS:LUC\%(pregnant:1.57 \pm 0.59 , non-pregnant: $1.77 \pm 0.80 ; \mathrm{p}=0.001$ ), delta neutrophil index (DNI) (pregnant: $2.07 \pm 0.51$, non-pregnant: $2.49 \pm 0.43 ; \mathrm{p}=$ 0.001 ); WBC (pregnant: $8.25 \pm 1.52 \times 103 / \mathrm{uL}$, non-pregnant: $7.31 \pm 1.64 \times 103$ / $\mathrm{uL} ; \mathrm{p}=0.001$ ); neutrophil \% (pregnant: $66.54 \pm 8.67$, non-pregnant: $62.0 \pm 9.31$; $\mathrm{p}=0.001$ ); monocyte $\%$ (pregnant: $4.87 \pm, 1.32$, non-pregnant: $5.27 \pm 1.5 ; \mathrm{p}$ $=0.001$ ); eosinophil $\%$ (pregnant: $1.67 \pm 0.39$, non-pregnant: $2.05 \pm 0.73 ; \mathrm{p}=$ 0.001 ); basophil \% (pregnant: $0.31 \pm 0.18$, non-pregnant: $0.41 \pm 0.04 ; \mathrm{p}=0.001$ ); lymphocyte \% (26.02 \pm 5.66 , non-pregnant: $28.49 \pm 4.68 ; \mathrm{p}=0.001)$ showed significant difference.

CONCLUSIONS:Large unstained cells are reported in routine complete blood cell (CBC) tests. LUC are large peroxidase-negative cells, which do not fit into other subtypes of leukocytes and they usually include virally activated lymphocytes, plasma cells, hairy cells, pediatric lymphocytes and peroxidase-negative lymphoblasts. In our study, LUC and DNI values were lower in pregnant women than non-pregnant women. Immune alterations with pregnancy may impair pathogen clearance, resulting in increased severity of disease for several pathogens. Therefore, we hypothesized that LUC value would be increased in infection and it can be a potential useful marker to differentiate infection. It can be suggested that there is resistance to inflammation during pregnancy.

Keywords: inflammatuary marker; $\mathrm{LUC} \%$; pregnanant

\section{O-099 \\ Biological variation of beta-trace protein, a novel marker for eGFR along with traditional markers}

Anil Baysoy ${ }^{1}$, Inanc Karakoyun ${ }^{1}$, Banu Isbilen Basok ${ }^{1}$, Fatma Demet Arslan¹, Ayfer Colak ${ }^{1}$, Can Duman ${ }^{2}$

${ }^{1}$ Department of Medical Biochemistry, Health Sciences University Tepecik

Training and Research Hospital, Izmir, Turkey

${ }^{2}$ Department of Medical Biochemistry, Izmir Democracy University, Izmir, Turkey

OBJECTIVES:Objective evaluation of a test result by the clinician, the establishment of an analytical performance goal or deciding the appropriateness of population-based reference range require the knowledge on the biological variation of the test. In the study, it is aimed to determine the biological variation of beta-trace protein (BTP), a relatively filtration marker for glomerular filtration rate (GFR) along with the conventional markers such as creatinine and cystatin c. MATERIALS and METHODS:Twenty-two participants aged between 25 and 57 were included in the study. "European Federation of Laboratory Medicine Biological Variation-WG" recommendations were followed. Whereas creatinine levels were analyzed using an autoanalyzer (AU5800,Beckman Coulter Inc.,USA), cystatin C and BTP were measured by nephelometric method 
(Atellica NEPH630 System,Siemens Healthineers,Germany). Intra (CVI) and inter-individual (CVG) biological variations for each parameter were calculated, reference change values (RCV), and individuality indexes were determined from these data.

RESULTS:CVA, CVI, and CVG values were as follows, respectively: 5.56/3.31/14.50 for creatinine; 3.48/3.15/12.24 for cystatin C, 5.37/9.91/14.36 for BTP. RCV values for creatinine, cystatin C, and BTP were calculated as $17.94 / 13.01 / 31.24$, while individuality indexes were found to be $0.23 / 0.26 / 0.69$, respectively.

CONCLUSIONS:To our knowledge, the study is the first study in literature in which the biological variation of BTP is determined. The closeness of CVI and CVG results for BTP might be interpreted as the molecule not having a substantial natural variation or significant individual characteristics. Because of the high individuality of creatinine and cystatin $\mathrm{C}$ tests, using RCV values instead of population-based reference ranges would be more useful in monitoring patients.

Keywords: Biological variation, Beta trace protein, Creatinine, Cystatin C, reference change value

\section{O-100}

Calculation of APTTandPT reference intervals from patient data and evaluation of preoperative test utilisation in surgical patients

Neslihan Cihan, Aysenur Macun Ayan, Ilknur Alkan Kusabbi, Mehmet Senes, Doğan Yucel

Department of Medical Biochemistry, Ankara Health Research and Training Hospital, Ankara, Turkey

OBJECTIVES:The purpose of this study was to verify the reference intervals of our own laboratory by indirect procedure for activated partial thromboplastin time (APTT) and prothrombin time (PT) and investigate whether preoperative coagulation test requests are necessary.

MATERIALS and METHODS:Bhattacharya procedure was used for determination of reference intervals from data of outpatient clinics between January 2017 and June 2019. We eliminated the test requests made by clinics of Child and Adult Emergency Department, Anesthesia and Reanimation, Obstretrics and Gynecology, Nephrology, Infectious Disase, Child and Adult Hemotology outpatients, inpatients and intensive care units. To determine the appropriate test utilisation, preoperative APTTandPT requests were used between July 2018 - June 2019 ( $\mathrm{n}=$ 4751). Cardiology, Cardiovascular surgery and Oncosurgery patients and repeated test requests were excluded. We evaluated preoperative test requests of APTT and PT by using reference intervals that we created from our own hospital data.

RESULTS:Reference intervals of APTT (sec) and PT (sec) for 1-3, 4-6, 7-9, 10$12,13-18$ age groups were $23.98-34.87$; $11.32-14.45,24.10-33.08 ; 11.69-14.37$, $24.45-34.67$; $11.73-14.38,24.87-33.90 ; 11.82-14.45,24.23-35.18 ; 11.87-14.42$, respectively. Adult reference intervals were for $18-39,40-49,50-59,60-69,70-$ 79,80 and above age groups were $24.06-33.63,11.56-14.87 ; 23.28-32.40,11.12-$ $14.07 ; \quad 23.88-11.09-14.38 ; \quad 23.62-32.55,11.17-14.65 ; 23.10-32.43,11.3-14.59$; $22.73-35.29,11.35-14.81$ respectively. In pediatric patients $77.1 \%$ of APTT and $61.4 \%$ PT results; in adult patients $75.8 \%$ of APTT and $60.9 \%$ of PT results were within the reference ranges.

CONCLUSIONS:A large proportion of preoperative coagulation tests are found within reference ranges and these test requests are mostly unnecessary, time consuming and high costly activities.

Keywords: preoperative tests, hemostasis, reference ranges

\section{O-101}

ICD code specific normal ranges are needed, particularly in total bilirubin in this case

Özgür Aydın

Kepez Public Hospital, Clinical Biochemistry Laboratory, Antalya

OBJECTIVES:Two healthy young patients admitted to the hospital to obtain a formal report of health. Their total and direct bilirubin levels were slightly higher than the upper reference level so a call was made to the laboratory to investigate the suspicious results.
MATERIALS and METHODS:Internal quality results are validated daily for each instrument in our laboratory and they are nearly perfect; while external quality results for the previous month were also satisfactory. The total and direct bilirubin analysis were repeated in another auto-analyzer but there was no change in the results..

RESULTS:These two individuals were recorded in the laboratory information system (LIS) with a specific ICD code Z02 namely "Encounter for administrative examination". Prior to medical health check, all nominees had to pass a written exam followed by a physical exam including a long distance running. A pubmed search with key words "sports" and "hemolysis" defined a rise in bilirubin levels of athletes.

CONCLUSIONS:The elevation in bilirubin is thought to be caused by mechanical factors, and named as "marching hemolysis". The literature suggests to use a dedicated reference range for total bilirubin concentration in relation to the group of athletes. This case is an evidence based application to use reference intervals dedicated to individuals defined by the mentioned specific ICD code. Unfortunately, we can only define reference intervals for age and gender with the LIS we currently use just like the ones we used before which is a limitation. Keywords: ICD codes, reference ranges

\section{0-102}

Pending laboratory tests at discharge in emergency department

Murat Alisik

Polatli State Hospital

OBJECTIVES:Emergency department (ED) is a department that requires rapid, accurate and effective intervention to patients. However, it is frequently encountered that patients are discharged before all tests are resulted. Laboratory test results pending at discharge (TPAD) from emergency department is a major patient safety concern and can have major adverse health outcomes. The goal of this study is to evaluate the TPAD ratios and factors that affect this situation in our hospital.

MATERIALS and METHODS:Two groups are established as TPAD and tests reported before discharge(TRBD) from the patients admitting to ED and having laboratory test requisition.

RESULTS:Total number of patients with test requisition were 13347, and total number of tests were 34104 .

TPAD ratio is detected as $16,4 \%$.TPAD is more frequent in biochemical tests $(19,5 \%)$, hormones $(42,4 \%)$, urine tests $(18,7 \%)$, cardiac markers $(21,4 \%)$ and coagulation tests $(27,1 \%)$, while it is less frequently encountered in hematology tests $(10 \%)$, blood gas analysis $(4,6 \%)$, and blood ethanol levels $(10,9 \%)$.

Consideration of delaying tests, regarding the determined test completion time for requestedfromED, showed $5,5 \%$ delayinTPAD whileitwas $1,7 \%$ in TRBD $(p<0,001)$. Regarding the referral status and reporting the tests before discharge of 370 results with elevated Troponin tests, TPAD ratio was $12,1 \%(\mathrm{n}=38)$ in nonreferred patients and 3,5\%(n=2) in referred ones.

CONCLUSIONS:Current study indicates that patient discharge ratios before evaluating the test results is high. This situation endangers the safety of both the physician and the patient. Discharging the patients before the laboratory test are reported, especially the troponin levels in myocardial infarction suspicion, may give rise to irreversible results.

Keywords: Pending tests, laboratory

O-103

\section{The effect of blood lactate levels on mortality in patients with sepsis}

Kamile Yücel ${ }^{1}$, Mehmet Selçuk Uluer ${ }^{2}$, Said Sami Erdem ${ }^{3}$

${ }^{1}$ Medical Biochemistry, KTO Karatay University School of Health Sciences, Konya, Turkey

${ }^{2}$ Department of Anesthesiology and Reanimation, Konya Training and Research Hospital, Konya, Turkey

33Department of Biochemistry, Konya Training and Research Hospital, Konya, Turkey

OBJECTIVES:Severe sepsis and septic shock are one of the major causes of mortality in intensive care units, and elevated blood lactate levels are an important indicator of mortality. In this study, we aimed to investigate the effect 
of blood lactate values on mortality rate of patients in intensive care unit. MATERIALS and METHODS:The files of 74 patients diagnosed with sepsis since 01/01/2018 in the Reanimation Intensive Care Unit of Konya Training and Research Hospital were investigated retrospectively.

RESULTS:The mean age was $67.1 \pm 15.2$ years in the group with mortality within 30 days (n: 45) and 64.9 \pm 20.1 years in the group without mortality (n: 29). There was no difference between the groups in terms of age and gender (respectively, $p=0.76$, $\mathrm{p}=0.88$ ). The mean duration of hospitalization in patients with sepsis was $12.3 \pm 11.1$ days and $60.8 \%$ of them died. In the group with mortality, the input lactate value was $4.2 \pm 3.9 \mathrm{mmol} / \mathrm{L}$ and the output lactate value was $7.3 \pm 5.4 \mathrm{mmol} / \mathrm{L}$. In patients who were discharged, the input lactate value was $2.5 \pm 1.7 \mathrm{mmol} / \mathrm{L}$ and the output lactate value was $2.3 \pm 3 \mathrm{mmol} / \mathrm{L}$. In our study, we found that the last measured lactate levels of the patients in the intensive care unit were higher than the first measured lactate levels in the group with mortality and this difference was statistically significant $(\mathrm{p}=0,000)$.

CONCLUSIONS:Lactate was an independent predictor of sepsis prognosis. Serial lactate monitoring helps to identify patients at high risk of developing mortality and is important for assessing the adequacy of treatment given to these patients. Keywords: Sepsis, mortality, lactate

\section{0-104}

The anti-inflammatory effects of orexin receptor antagonist on endotoxemia induced sepsis model

Meltem Kolgazi ${ }^{1}$, Evren Kılınç ${ }^{2}$, Sümeyye Çilingir

${ }^{1}$ Acibadem Mehmet Ali Aydinlar University, School of Medicine, Department of Physiology, Ataşehir/Istanbul, Turkey

${ }^{2}$ Acibadem Mehmet Ali Aydinlar University, School of Medicine, Department of Biophysics, Ataşehir/Istanbul, Turkey

OBJECTIVES:Inflammatory diseases, including sepsis, are often accompanied by loss of appetite. As an orexigenic peptite, orexin increases appetite; however little is known about its role in sepsis related inflammatory conditions. Thus, the aim of the study is to investigate the role of orexin in Escherichia coli lipopolysaccharide (LPS) induced endotoxemia by using its dual receptor antagonist almorexant

MATERIALS and METHODS:Sprague Dawley rats (male=female; 250-300g) were used: (1) Control and (2) Endotoxemia (E) groups were treated with saline; (3) $\mathrm{E}+$ orexin antagonist group was treated with almorexant $(30 \mathrm{mg} / \mathrm{kg}$ ip) for 3 days. On the 4th day, saline (control group) or LPS (others) was injected. Six hours after LPS injection, rats were sacrificed; their trunk blood, duodenum, stomach, liver, colon and kidney samples were collected. Tissue samples were analyzed for myeloperoxidase (MPO) activity, malondialdehyde (MDA) and glutathione (GSH) levels and microscopic damage was scored. Cortisol, tumor necrosis factor (TNF)- $\alpha$, Interleukin (IL)-1 $\beta$ and IL-6 levels were measured in serum samples.

RESULTS:Endotoxemia increased MPO activity, MDA levels in all tissues and caused GSH depletion. MPO activity and MDA levels in all tissues and, cortisol, TNF- $\alpha$, IL-1 $\beta$ and IL-6 levels in serum were decreased with almorexant injection compared with the endotoxemia group. Microscopic damage scores also reduced. However, almorexant treatment could not prevent GSH depletion induced by endotoxemia.

CONCLUSIONS:The results of our research showed that almorexant has antiinflammatory effects on LPS induced sepsis. Probably, dual orexin receptor antagonist, almorexant showed its anti-inflammatory effects by inhibiting tissue neutrophil infiltration and preventing lipid peroxidation.

Keywords: Orexin, Almorexant, Sepsis, Endotoxemia, inflammation

\section{O-105 \\ Correlation of CRP with blood-based inflammatory markers; large cohort study}

Sibel Soylemez ${ }^{1}$, Durmus Ayan ${ }^{2}$

${ }^{1}$ Department of Medical Biochemistry, Gazi University, Ankara, Turkey ${ }^{2}$ Department of Medical Biochemistry, Amasya Public Health Laboratory and Department of Medical Biochemistry, Amasya University Sabuncuoglu Serefeddin Research and Training Hospital, Amasya, Turkey

OBJECTIVES:The aim of the study is to examine the relationship between CRP and several blood-based markers (neutrophil/lymphocyte, platelet/lymphocyte, lymphocyte/monocyte, monocyte/HDL ratio), which have recently become popular in a large population of patients.

MATERIALS and METHODS:Samples were taken at Amasya Central Public Health Laboratory between 01.01.2018-31.12.2018 and the results of $n=26,691$ (18,243 females, 8,448 males) patients were screened retrospectively. The correlation between CRP and neutrophil/lymphocyte ratio (NLR), platelet/ lymphocyte ratio (PLR), lymphocyte/monocyte ratio (LMR), monocyte/HDL ratio (MHR) were determined using the SPSS 15 for Windows program.

RESULTS:According to the results of the study, there was a positive correlation (r: $0.301, \mathrm{p}<0.01, \mathrm{r}: 0.180, \mathrm{p}<0.01, \mathrm{r}: 0.305, \mathrm{p}<0.01$, respectively) between CRP and NLR, PLR, and MHR, while there was a negative correlation (respectively: $\mathrm{r}:-0.224, \mathrm{p}<0.01, \mathrm{r}:-0.102 \mathrm{p}<0.01)$ between CRP and LMR and HDL.

CONCLUSIONS:Based on the positive correlation (excluding LMR) of the rates of hematologic parameters and CRP, a marker of classical inflammation, we think that these rates can be used as a supportive marker for inflammation in patients under CRP follow-up. However, we believe that these rates should be supported by further studies to be conducted in various patient groups. Keywords: inflammatory markers

\section{O-106}

Serum cytokine and complement levels in amyotrophic lateral sclerosis and their association with LRP antibody positivity

\section{Murat Giriș $^{1}$, Yeșim Parman ${ }^{2}$}

${ }^{1}$ Istanbul University, Aziz Sancar Institue of Experimental Medicine, Turkey ${ }^{2}$ Istanbul University, Istanbul Medical Faculty, Department of Neurology, Turkey

OBJECTIVES:It has been known that neuroinflammation plays an important role in the pathophysiology of amyotrophic lateral sclerosis (ALS), and few antineuronal antibodies have been detected in some studies. The aim of this study was to investigate the role of primary T helper subset Th1 (IFN-gama), Th2 (IL4) and Th17 (IL-17) cytokines and their association with complement factors. MATERIALS and METHODS:25 patients with ALS (mean age 56.5 \pm 6.9 years; 16 males $/ 9$ females) and 25 healthy controls ( $58.7 \pm 8.5$ years; 14 males/11 females) were included in the study. Serum C1q, C3, IL-4, IL-17 and IFNgama levels were measured by ELISA and LRP4-antibody was demonstrated by immunofluorescence method performed with HEK cells transfected with the plasmid encoding LRP4.

RESULTS:Serum C1q and C3 levels were lower and IL-17 levels were higher in ALS patients compared to healthy controls. There was no significant difference between IL-4 and IFN-gama levels. In spinal-onset ALS patients serum C1q and C3 levels were higher than those with bulbar onset. LRP4 antibody was found in 4 cases. All patients with LRP4 antibody positive sera had spinal onset. C1q and C3 levels were significantly higher in the LRP4 antibody positive sera. In primer neuron culture studies, only LRP4 antibody-positive ALS serum IgG molecules binded neurons.

CONCLUSIONS:Our results support that the predominant role of Th17-type immunity in ALS. The increase of complement factors in spinal-onset cases suggests that the complement system is involved in pathogenesis of these patients. Conceivably, LRP4 antibodies might bind LRP4-expressing motor neurons thereby activating the complement system and thus contributing to motor neuron destruction.

Keywords: Amyotrophic Lateral Sclerosis, complement factors, Cytokine, LRP4 antibody 


\section{0-107}

The relationship between standard sedo-analgesia implementation and serum procalcitonin levels in intensive care unit

Yeșim Güvenç Demirağcı ${ }^{1}$, Idil Tekïn², Orhan Kılıç², Demet Aydın²

${ }^{1}$ Department of Medical Biochemistry, Manisa Celal Bayar University Faculty of Medicine, Manisa, Turkey

${ }^{2}$ Department of Anesthesiology and Reanimation, Intensive Care Unit,Manisa Celal Bayar University Faculty of Medicine, Manisa Turkey

OBJECTIVES:Inflammatory pain is a common symptom in intensive care unit (ICU) patients. Procalcitonin levels increase in systemic inflammation. The aim of this study was to determine the relationship between serum procalcitonin levels and standard sedo-analgesia in the control of inflammation-related pain levels in the ICU patients.

MATERIALS and METHODS:The study included 69 patients hospitalized to the Anaesthesiology and Reanimation ICU of Manisa Celal Bayar University. Patients are divided into two groups; Group 1 $(\mathrm{n}=36)$ : within two months before, and Group 2(n = 33): within two months after standard sedo-analgesia protocol was implemented. Before the implementation of the sedo-analgesia protocol, patients were treated based on the subjective evaluation of physicians and nurses at irregular intervals. After the implementation of sedo-analgesia protocol, pain and sedation requirements, were evaluated regularly with reliable scales, and treated with appropriate prescribed drugs and doses. Serum procalcitonin levels were daily measured for five days. Serum procalcitonin levels were daily measured for five days with Cobas e411 autoanalyser.

RESULTS:Procalcitonin difference between day 1 and day 5 was analyzed in both groups and no statistically significant difference was found between Group 1 and Group $2(\mathrm{p}=0.41)$. When the 5 -day procalcitonin values of both groups were compared, no strong correlation was observed $(\mathrm{r}=0.412,-0.150,0.053$, $0.365,0.291$, respectively).

CONCLUSIONS:Procalcitonin did not show a different course in the five-day follow-up with the start of our sedo-analgesia protocol. Thus, we conclude that procalcitonin may not be used as a biomarker to monitor the standart sedoanalgesia protocol.

Keywords: Pain, Procalcitonin, Sedation, Analgesia, Inflammation

\section{O-108 \\ Midkine can not be accepted as a new biomarker for the diagnosis and the treatment of unexplained female infertility}

\section{Mine Erguven ${ }^{1}$, Tulay Irez ${ }^{2}$}

${ }^{1}$ Department of Medical Biochemistry, Faculty of Medicine, İstanbul Aydın University, Istanbul, Turkey.

${ }^{2}$ Department of Histology and Embryology, Faculty of Medicine, Biruni University, Istanbul, Turkey.

OBJECTIVES:This study aimed to investigate whether a growth factor and a cytokine midkine (MK) can be a new biomarker for the diagnosis and the treatment of unexplained female infertility (UFI) cases.

MATERIALS and METHODS:Serum (S), follicle fluid (FF) and cumulus cells (CCs) of the patients aged 20-45 years, diagnosed with male factor (MF), UFI and polycystic ovary syndrome (PCOS) and undergoing Intracytoplasmic Sperm Injection (ICSI) procedure were used. The Anti-Müllerian hormone (AMH) and MK levels with other hormone levels, the oocyte and embryo qualities, the fertilization and pregnancy rates, and cumulus cells (Number, ultrastructure, and apoptosis) were evaluated. Student-T-test was performed and $\mathrm{p}<0.05$ was considered statistically significant.

RESULTS:The lowest and highest numbers of CCs were found at UFI and PCOS, respectively $(\mathrm{p}<0.05)$. The lowest viability rate with the highest apoptosis rate was determined at PCOS $(\mathrm{p}<0.05)$. The lowest apoptosis rate with the highest viability rate was evaluated at MF $(\mathrm{p}<0.05)$. The ultrastructural evaluation revealed that there were widespread autophagic vacuoles at PCOS and lipid droplets at UFI with MF. $\mathrm{CCs}$ with apoptotic appearance was frequently detected at PCOS. Highest AMH and MK levels (S, FF) were found at PCOS; however, the lowest levels of them were detected at UFI $(p<0.05)$. These values found at UFI were similar to MF $(p>0.05)$. CONCLUSIONS:MK can not be accepted as a new biomarker for the diagnosis and treatment of UFI.

Keywords: Unexplained female infertility, midkine, anti-müllerian hormone, polycystic ovary syndrome, cumulus.
0-109

Perspective of C-Peptide from diabetes window

Fikret Akyurek, Saadet Kader

Department of Biochemistry, Faculty of Medicine, Selçuk University, Selçuklu, Konya, Turkey.

OBJECTIVES:C-peptide indicates endogenous insulin production. It is a good marker for $\beta$-cell capacity. In recent years, the use of $\beta$-cell capacity for insulin use in the treatment of Type $2 \mathrm{DM}$ has been recommended. According to the C-peptide level, the use of oral antidiabetics has been prescribed. C-peptide is used for monitoring pancreatic capacity. In this study, we aimed to emphasize the importance of C-peptide in the of DM. MATERIALS and METHODS:The number of C-peptide tests studied by the biochemistry laboratory of our hospital in the last 5 years were classified according to years. Subsequently, the annual changes were grouped as below 1.1, 1.1-4.4 and > 4.4 and $\%$ ratios were found.

RESULTS:6794 C-peptide analyzes have been performed in our laboratory in the last 5 years. Percentage distribution of patients by years was found respectively as $11.2,11.4,18.0,23.2,36.3$. The annual percentage distribution of patients with C-peptide $<1.1$ was respectively $23.2,42.1,19.7,35.0,10.9$. The ratio of patients with C-peptide between 1.1-4.4 were 56.0, 31.4, 62.7, 52.1, 73.6 per years. The ratio of patients with C-peptide> 4.4 to overall patients in the same year was 20.8, 26.5, 17.6, 12.9, 15.4 .

CONCLUSIONS:There was a continuous increase in the number of test requests. The C-peptide is used not only for DM classification but also for the follow-up of DM patients. We think that this will reduce the need for parenteral insulin treatment. We think that the clinical laboratory planning should be made accordingly. Keywords: C-peptide, DM, $\beta$-cell

\section{O-110}

Development and validation of a biosensor for measurement of serum hypoxia-inducible factor-1

$\underline{\text { Zihni Onur Uygun }}^{1}$, Hilmiye Deniz Ertuğrul Uygun², Sinem Nur Şengöz Coşkun ${ }^{3}$, Yasemin Akçay ${ }^{1}$, Şevki Çetinkalp ${ }^{3}$, Ferhan Sağın ${ }^{1}$ ${ }^{1}$ Medical Biochemistry Dept., Faculty of Medicine, Ege University, İzmir, Turkey ${ }^{2}$ Center for Production and Application of Electronic Materials, Dokuz Eylül University, İzmir, Turkey

${ }^{3}$ Endocrinology Dept., Faculty of Medicine, Ege University, İzmir, Turkey

OBJECTIVES:Normal oxygen delivery is essential for survival. Hypoxia, which is a common feature of various pathological conditions, ranging from cancer to inflammatory diseases, occurs when normal oxygen delivery is altered by an imbalance between cellular oxygen demand and tissue oxygen supply. Among the intricate mechanisms organisms have developed to maintain oxygen homeostasis, a family of hypoxia-inducible transcription factors (HIFs), are found to be the main regulator adaptive cellular response to hypoxia. Although ELISA can be used for its measurement, the lability of the protein and length of the analysis ( $>5$ hours) pose limitations. Thus, our aim is to develop an electrochemical impedance spectroscopy (EIS) based biosensor system for quick and reliable measurement of HIF-1 $\alpha$ in tissue.

MATERIALS and METHODS:HIF-1alpha antibodies have been used as a biota receptor. For immobilization, the electrode was first modified with albumin, followed by PAMAM. The new biosensor was compared with the conventional ELISA method.

RESULTS:Based on the chronoimpedance data, total analysis time for EIS was chosen as 15 minutes. Calibration curve was constructed by locating electron transfer resistance on y-axis and HIF1 concentration on x-axis, between 50-1000 $\mathrm{pg} / \mathrm{mL}$. LOD and LOQ of the biosensor were calculated as $14.45 \mathrm{pg} / \mathrm{mL}$ and $43.8 \mathrm{pg} / \mathrm{mL}$, respectively. The new biosensor showed very good correlation when compared with the conventional ELISA method $(\mathrm{R} 2=0.99649)$.

CONCLUSIONS:We developed and analytically validated a biosensor system to measure HIF-1 $\alpha$ in serum. This new biosensor promises more timely and accurate measurements in determining the tissue oxygenation in patients who have hypoxia related conditions such as diabetic foot.

Keywords: hypoxia inducible factor 1 alpha, biosensor, impedance, PAMAM 
O-111

A fast and convenient UPLC - MSMS method for routine analysis of GALT activity from dried blood spot

Muhammet Topbaș ${ }^{1}$, Erhan Canbay ${ }^{1}$, Ebru Demirel Sezer ${ }^{1}$, Sema Kalkan Uçar², Mahmut Çoker $^{2}$, Eser Yıldırım Sözmen ${ }^{1}$

${ }^{1}$ Department of Medical Biochemistry, Faculty of Medicine, Ege University,

Izmir, Turkey

${ }^{2}$ Department of Pediatric Metabolic Diseases, Faculty of Medicine, Ege

University, Izmir, Turkey

OBJECTIVES:Galactosemia is a disorder of carbohydrate metabolism most commonly caused by galactose-1-phosphate uridylyltransferase(GALT) deficiency.Currently,GALT deficiency screening is performed by fluorometric method from dry blood spots(DBS) and comfirmed by LC/ MSMS from whole blood samples. The aim of our study is to develop a fast,low cost,reliable LC/ MSMS based method for detection of GALT activity from DBS which does not need whole blood samples for verification and to compare the method performance with a commercial fluorometric neonatal GALT kit.

MATERIALS and METHODS:In the developed method,ACQUITY UPLC HSS-T3,2.7 $\mu \mathrm{m}, 2,1 \times 50 \mathrm{~mm}$ column was used as the stationary phase and $5 \mathrm{mM}$ ammonium formate in acetonitrile/water $50 / 50 \%$ was used as the mobile phase. Injection volume was $5 \mu \mathrm{L}$ while flow rate was $0.4 \mathrm{~mL} /$ minute.Mass spectrums were determined with Waters Xevo TQD MS/MS system.

RESULTS:The method has been fully validated to ensure good selectivity,a satisfactory detection limit at $6.2 \mathrm{nM}$ for UDP-Galactose, acceptable intra- and inter-day accuracy and high precision. A linear response function was established for the range of concentrations between $0.05-100 \mu \mathrm{M}\left(\mathrm{R}^{2}=0.9992\right)$ for ${ }^{13} \mathrm{C} 6-$ UDPGalactose.Controls' enzyme activity levels were clearly distinguishable from patients' levels $\left(\mathrm{p}<10^{7}\right)$ with a mean value of $42.29 \pm 19.73 \mu \mathrm{mol} / \mathrm{gHb} / \mathrm{h}$ $(\mathrm{n}=50)$ for controls and $0.03 \pm 0.025 \mu \mathrm{mol} / \mathrm{gHb} / \mathrm{h}$ for patients $(\mathrm{n}=7)$. Recovery was found as $88 \%$ for low QC and $89 \%$ for high QC whereas matrix effect was found as $88 \%$ for low QC and $102 \%$ for high QC.

CONCLUSIONS:This fast, accurate, reliable and sensitive method to analyze GALT levels with LC-MS/MS system in DBS could contribute to facilitate a national newborn screening program in Turkey.

Keywords: GALT, DBS, LCMSMS, newborn screening, validation

\section{O-112}

Magnetic bead based electrochemical food and enzyme activity analysis by using SPE dependent immunosensors

Ebru Saatçi, Tuğba Özkaya Ferak, Mahmood Taha Noori Al Sadoon

Department of Biology, Erciyes University, Kayseri, Turkey

OBJECTIVES:Gathering magnetic beads (MBs) and screen printed electrode technology allows a highly sensitive and innovative methodology for the development of amperometric immunosensors. These researchs purpose are the detection of $\beta$-casomorphine-7 peptide in cheese rennet and analyze the substrate effect on HRP-based amperometric immunosensors by using this combined technology.

MATERIALS and METHODS:Both study were based on direct and competitive immunosensor protocols in which antigen-antibody and labeled enzyme immunoassay procedure was followed. HRP substrates ABTS and TMB were used to measure the HRP activity as the last step of the amperometric immunosensor analysis. Signal of the biosensors was in nano amper (nA) range. RESULTS:For BCM-7 detection, competitive type immunosensor sensitivity was found in $\mathrm{ng} / \mathrm{ml}$ range and the detection limits were found as $0.5-200 \mathrm{ng} / \mathrm{ml}$. BCM-7 detection in different commercially available cheese rennets was also done. For HRP-based enzymatic sensor development, it is found that TMB is more sensitive than ABTS as the substrate of the HRP.

CONCLUSIONS:BCM-7 detection in cheese rennet with MB-SPE based immunosensor is the first study in the literature. For the other study, HRP activity detection with immunosensor type biosensor by using different substrates gives us the best substrate for HRP activity determinations in amperometric detection. These studies were supported by Erciyes University Scientific Research Projects Unit under the code of FYL-2018-8413 and Tübitak 1509, 9130058, PrintECELISA project.

Keywords: Amperometric immunosensor, BCM-7, HRP, ABTS, TMB
O-113

Transcriptomic meta-analysis in pancreatic ductal adenocarcinoma reveals therapeutic targets and diagnostic biomarkers

Sevcan Atay

Department of Medical Biochemistry, Ege University Faculty of Medicine, Izmir, Turkey

OBJECTIVES:Pancreatic ductal adenocarcinoma (PDAC) is the most common form of pancreatic cancer, which has the highest mortality rate of all solid tumors. The absence of an effective screening process and distinctive symptoms, causes a delay in diagnosis. Traditional chemotherapy and curative surgery have limited benefits on patient survival. Enzymes are one of the most important groups of drug targets and are preferred markers for the detection of various diseases. This study aims to identify up-regulated genes encoding enzymes in PDAC to suggest novel therapeutic targets for more effective treatments to be developed and diagnostic biomarkers for PDAC

MATERIALS and METHODS:NCBI Gene Expression Omnibus (GEO) was searched for datasets using keywords 'pancreatic ductal adenocarcinoma'. The inclusion criteria were i) Gene expression microarray data, ii) human-derived pancreatic ductal adenocarcinoma tissues and normal pancreatic tissue samples. All data processing and integration procedures were performed using ExAtlas. The false discovery rate is less than 0.05 , and the change of gene expression is $\geq 10$-fold were considered significant. The up-regulated enzyme-coding genes were detected in the differentially expressed gene list.

RESULTS:The random effect integrative meta-analysis of five submissions (GSE46234, GSE19280, GSE43795, GSE41368, and GSE71989) containing 24 tumor-normal tissue pairs revealed 22 up-regulated genes, two of which encoding enzymes. The enzyme-coding genes with 10 -fold differential expression compared to the controls were SULF1 (sulfatase, fold change=22.135) and KYNU (kynureninase, fold change=10.716).

CONCLUSIONS:The results of this study suggest that sulfatase and kynureninase may have the potential to become diagnostic biomarkers and therapeutic targets for PDAC, which merits further investigation.

Keywords: Pancreatic Ductal Adenocarcinoma, microarray, meta-analysis, enzyme, gene expression.

\section{O-114}

\section{Assessment of Vitamin D levels in Şanlıurfa region}

Nihayet Bayraktar, Oruç Aslan, Müjgan Ercan, Ekrem Yaman,

Mesut Yardimciel, Melek Alan, Ataman Gönel

Harran University Medical School, Department of Medical Biochmistry, Şanliurfa

OBJECTIVES:The aim of this study was to determine serum vitamin D levels in Şanliurfa region and to investigate the existence of difference between its level saccordingtoage, sex and season.

MATERIALS and METHODS:Scanning the findings of serum 25-hydroxy vitamin D at software system of Biochemistry Laboratory, Education and Research Hospital, Harran University was applied during the period 01.01.2018 and 01.06.2019. Serum 25-hydroxy vitamin D level had determined by LCMS8045 liquid chromatography mass spectrometer auto-analyzer. SPSS version 21 program was used to evaluate normality test with Kolmogorov-Smimov test. Descriptive statistics were expressed as median (min-max) since the data were nonparemetic. Mann-Whitney U test andKrusskal-Wallis test were used fo rstatistical difference among the studied groups. Significance level was accepted as $\mathrm{P}<0.05$. Intra-laboratory variation coefficients (CV\%) of all methods used were $<3 \%$.

RESULTS:In our retrospective study, vitamin D deficiency was found in $68.21 \%$ of 6182 patients. In comparison the results according to gender, age, and season, a significant difference $(\mathrm{p}<0.001)$ was found in serum vitamin $\mathrm{D}$ levels and this was in accordance with the literature.

CONCLUSIONS:As a result, In Sanliurfa, the reference range of vitamin D level was determined according to age, sex and seasonal parameters. Considering the influencing factors, we strongly recommend checking serum vitamin $25(\mathrm{OH})$ -D during annual controls and to raise public awareness about importance of sunbathing to prevent vitamin D deficiency.

Keywords: Vitamin 25-OH, Age, Sex 


\section{O-115}

The role of HDL-associated MPO and PON-1 for coronary artery disease in Hashimoto Thyroiditis

Emre Avci' ${ }^{1}$, Gizem Uncu' ${ }^{1}$, Gulcin Alp Avci ${ }^{1}$, Alpaslan Karabulut ${ }^{2}$,

Cumhur Bilgi ${ }^{3}$

${ }^{1}$ Hitit University, Faculty of Arts and Sciences, Department of Molecular

Biology and Genetics, Corum,Turkey

${ }^{2}$ Hitit University, Faculty of Medicine, Department of Internal

Medicine,Corum,Turkey

${ }^{3}$ Yüksek İhtisas University, Faculty of Medicine, Department of Medical

Biochemistry,Ankara,Turkey

OBJECTIVES:Hashimoto's thyroiditis is an autoimmune disease of the thyroid gland.Free radicals have been reported to be responsible for the complications observed in the pathogenesis of thyroid diseases and in the later stages of the disease.MPO, which is released during inflammation, is an oxidative enzyme present in phagocytes.MPO could be a key element responsible for oxidative damage in the artery wall.PON-1, which is one of the molecules that play a role in oxidant balance, is an enzyme that has the role of inhibiting lipoprotein oxidation by hydrolyzing lipid peroxides in oxidized LDL structure. We investigated the role of HDL-associated MPO and PON-1 in patients with HT in terms of coronary artery disease

MATERIALS and METHODS:Our study group consisted of 54 patients with Hashimato diagnosis and 28 healty individuals as control group.MPO and PON1 levels were determined spectrophotometrically

RESULTS:When the study groups were evaluated,PON1 levels were significantly lower in patients with hashimato thyroiditis than healthy subjects $(\mathrm{p}<0.05, \mathrm{p}=0.032)$. When the study groups were evaluated, MPO levels were significantly higher in patients with hashimato thyroiditis than the control group $(\mathrm{p}<0.05, \mathrm{p}=0.001)$. A negative correlation was obtained between MPO and PON1(r=-0.685)

CONCLUSIONS:The decrease in PON1 activity and increase in MPO activity due to hypothyroid effect increases lipid peroxide formation and accelerates oxiLDL formation,which leads to decrease in antioxidant capacity and development of atherosclerosis.Since oxidative stress in thyroid diseases is also responsible for the complications observed in the later stages of the disease,we think that important data were obtained with this study in terms of both diagnosis and treatment

Keywords: Hashimoto Troiditis, MPO,PON-1,HDL

\section{O-116}

Drug-induced (quinine) acute hepatitis with high level of serum vitamin B12

Özlem Özün ${ }^{1}$, Ferhat Demirci²

${ }^{1}$ Özlem Özün, University of Health Sciences Suat Seren Chest Diseases and Surgery Training and Research Hospital, Medical Biochemistry Laboratory, Izmir, Turkey

${ }^{2}$ Ferhat Demirci, University of Health Sciences Suat Seren Chest Diseases and Surgery Training and Research Hospital, Medical Biochemistry

Laboratory,Izmir,Turkey

OBJECTIVES:Acute hepatitis; viruses, drugs, alcohol, metabolic diseases, toxins caused by liver cell necrosis and inflammation of the liver. Since liver plays an important role in the metabolism of drugs, drugs are one of the most common causes of toxic liver damage. The diagnosis of drug-induced hepatitis is usually made by ruling out other causes and it should be remembered in the patient's history.

In this case, a patient who presented to the infectious diseases clinic with fatigue was presented.

MATERIALS and METHODS:Drug-induced liver damage (IBCT) is one of the most common causes of hepatotoxicity

RESULTS:In liver pathologies, ALT, AST, ALP, GGT and bilirubin tests, which are described as liver function tests, are expected to increase or decrease according to the severity of the clinic. What is striking in this patient is the change in vitamin B12 measurements. Although it was initially thought to be the interference of quinine in immunassay methods, a B12-quinine interference was not found in the literature review.

CONCLUSIONS:The elevation of vitamin B12 in liver cell damage suggests that it can be used as a marker in the diagnosis and / or treatment of acute hepatitis. Furthermore, as observed in cirrhosis cases, rapid decrease after elevation in AST and ALT enzymes indicates a poor prognosis and a prolonged tendency to increase in B12 levels may indicate a poor prognosis.

Keywords: Acute hepatitis, vitamin B12, quinine 